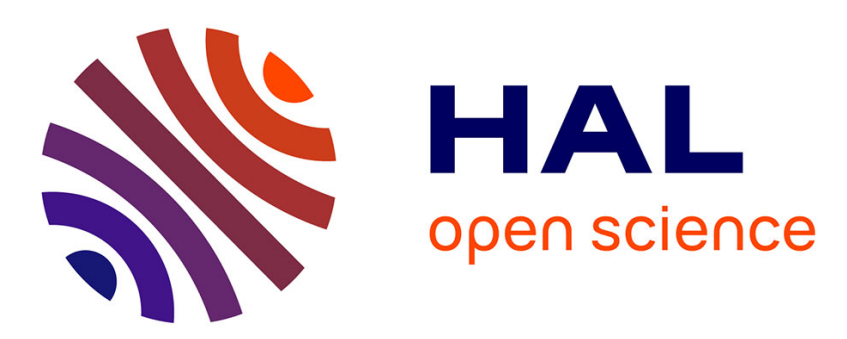

\title{
Optimization of the shape of regions supporting boundary conditions
}

Charles Dapogny, Nicolas Lebbe, Edouard Oudet

\section{To cite this version:}

Charles Dapogny, Nicolas Lebbe, Edouard Oudet. Optimization of the shape of regions supporting boundary conditions. Numerische Mathematik, 2020, 146, pp.51-104. 10.1007/s00211-020-01140-0 . hal-02064477

\section{HAL Id: hal-02064477 \\ https://hal.science/hal-02064477}

Submitted on 11 Mar 2019

HAL is a multi-disciplinary open access archive for the deposit and dissemination of scientific research documents, whether they are published or not. The documents may come from teaching and research institutions in France or abroad, or from public or private research centers.
L'archive ouverte pluridisciplinaire HAL, est destinée au dépôt et à la diffusion de documents scientifiques de niveau recherche, publiés ou non, émanant des établissements d'enseignement et de recherche français ou étrangers, des laboratoires publics ou privés. 


\title{
OPTIMIZATION OF THE SHAPE OF REGIONS SUPPORTING BOUNDARY CONDITIONS
}

\author{
C. DAPOGNY ${ }^{1}$, N. LEBBE ${ }^{1,2}$, E. OUDET ${ }^{1}$ \\ 1 Univ. Grenoble Alpes, CNRS, Grenoble INP ${ }^{1}$, LJK, 38000 Grenoble, France \\ 2 Univ. Grenoble Alpes, CEA, LETI, F38000 Grenoble, France
}

\begin{abstract}
This article deals with the optimization of the shape of the regions assigned to different types of boundary conditions in the definition of a 'physical' partial differential equation. At first, we analyze a model situation involving the solution $u_{\Omega}$ to a Laplace equation in a domain $\Omega$; the boundary $\partial \Omega$ is divided into three parts $\Gamma_{D}, \Gamma$ and $\Gamma_{N}$, supporting respectively homogeneous Dirichlet, homogeneous Neumann and inhomogeneous Neumann boundary conditions. The shape derivative $J^{\prime}(\Omega)(\theta)$ of a general objective function $J(\Omega)$ of the domain is calculated in the framework of Hadamard's method when the considered deformations $\theta$ are allowed to modify the geometry of $\Gamma_{D}, \Gamma$ and $\Gamma_{N}$ (i.e. $\theta$ does not vanish on the boundary of these regions). The structure of this shape derivative turns out to depend very much on the regularity of $u_{\Omega}$ near the boundaries of the regions $\Gamma_{D}, \Gamma$ and $\Gamma_{N}$. For this reason, in particular, $J^{\prime}(\Omega)$ is difficult to calculate and to evaluate numerically when the transition $\overline{\Gamma_{D}} \cap \bar{\Gamma}$ between homogeneous Dirichlet and homogeneous Neumann boundary conditions is subject to optimization. To overcome this difficulty, an approximation method is proposed, in which the considered 'exact' Laplace equation with mixed boundary conditions is replaced with a 'smoothed' version, featuring Robin boundary conditions on the whole boundary $\partial \Omega$ with coefficients depending on a small parameter $\varepsilon$. We prove the consistency of this approach in our model context: the approximate objective function $J_{\varepsilon}(\Omega)$ and its shape derivative converge to their exact counterparts as $\varepsilon$ vanishes. Although it is rigorously justified only in a model problem, this approximation methodology may be adapted to many more complex situations, for example in three space dimensions, or in the context of the linearized elasticity system. Various numerical examples are eventually presented in order to appraise the efficiency of the proposed approximation process.
\end{abstract}

\section{CONTENTS}

1. Introduction

2. Optimization of a function of the domain allowing variations of the regions supporting boundary conditions

2.1. Presentation of the model physical problem and notations

2.2. The shape optimization problem

2.3. A brief account of the regularity of $u_{\Omega}$

3. Shape derivatives of the functional $J(\Omega)$

3.1. Calculation of the shape derivative of $J(\Omega)$ when only the homogeneous Neumann inhomogeneous Neumann transition is optimized

3.2. Calculation of the shape derivative of $J(\Omega)$ when only the Dirichlet-homogeneous Neumann transition is optimized

4. An approximate shape optimization problem to deal with the Dirichlet-Neumann transition

4.1. About the signed distance function to a subset on a manifold

4.2. The smoothed setting

4.3. The approximate shape optimization problem

4.4. Study of the convergence of the approximate model to the exact problem

\footnotetext{
${ }^{1}$ Institute of Engineering Univ. Grenoble Alpes
} 
5. Numerical illustrations

5.1. An academic, validation example 25

5.2. Optimization of the repartition of clamps and locators on the boundary of an elastic structure 27

5.3. Joint optimization of the shape and the regions supporting different types of boundary conditions

6. Conclusions and perspectives

Appendix A. Some facts from tangential calculus 36

\section{INTRODUCTION}

Stimulated by the multiple prospects offered by fields so diverse as structural engineering, fluid mechanics, electromagnetism or biology, to name a few, shape and topology optimization techniques have aroused much enthusiasm in the scientific and industrial communities; see for instance [6, 12, 31, 45] for reference monographs, and $[22,34]$ for an account of recent developments and research perspectives.

Shape optimization problems consist in minimizing a given objective function $J(\Omega)$ of the domain $\Omega$, possibly under some constraints. In most realistic applications, $J(\Omega)$ depends on the optimized shape $\Omega$ via a state $u_{\Omega}$ arising as the solution to a boundary value problem for a certain partial differential equation posed on $\Omega$, which characterizes its physical situation.

Quite often, it turns out that only one part of the boundary $\partial \Omega$ of the shape is subject to optimization, which is associated to one single type of boundary conditions for the state $u_{\Omega}$ in the underlying physical partial differential equation, while the remaining regions are not meant to be modified. For example, in structural design, where $u_{\Omega}$ is the displacement of the structure and is the solution to the linearized elasticity system, it is customary to optimize only the traction-free part of $\partial \Omega$ (i.e. that bearing homogeneous Neumann boundary conditions). Likewise, in fluid applications (where $u_{\Omega}$ is the velocity of the fluid, solution to the Stokes or Navier-Stokes equations), one is often interested in optimizing only the region of $\partial \Omega$ supporting no-slip (that is, homogeneous Dirichlet) boundary conditions.

A little surprisingly, the dependence of a given performance criterion $J(\Omega)$ with respect to the relative locations of regions accounting for different types of boundary conditions has been relatively seldom investigated in shape and topology optimization. Yet, situations where it is desirable to optimize not only the overall shape of $\Omega$ but also the repartition of the zones on $\partial \Omega$ bearing different types of boundary conditions are multiple in concrete applications. Let us mention a few of them:

- When the objective criterion involves thermal effects inside the optimized shape $\Omega, u_{\Omega}$ is the temperature, solution to the stationary heat equation. The regions of $\partial \Omega$ associated to Dirichlet boundary conditions are those where a known temperature profile is imposed, while Neumann boundary conditions account for heat injection. It may be desirable to investigate the regions where heat should enter the medium $\Omega$ (or those which should be kept at fixed temperature) in order to minimize, for instance, the mean temperature inside $\Omega$, or its variance; see for instance [10] about such physical applications.

- In the context of linearly elastic structures, (homogeneous) Dirichlet boundary conditions account for the regions of $\partial \Omega$ where the structure is fixed, while inhomogeneous (resp. homogeneous) Neumann boundary conditions correspond to regions of $\partial \Omega$ where external loads are applied (resp. to tractionfree regions). It may be of great interest to optimize the design of fixations [49], or the places where loads should be applied. One interesting application of this idea concerns the optimization of a clamping-locator fixture system (a very brief outline of the stakes of this subject is provided in Section 5.2 below); see for instance [41] in the framework of density-based topology optimization methods, or the contributions [36, 50], relying on genetic algorithms or artificial neural networks. More recently, in $[56,57]$ the authors present an adapted level set method for the joint optimization of the shape of an elastic structure $\Omega$ and of the region of its boundary $\partial \Omega$ where it should be fixed; see also [58] for analogous simultaneous optimization problems of the structural shape and of the support region using parametrized level set functions and B-spline finite element methods. 
- In acoustic applications, $u_{\Omega}$ is the solution to the time-harmonic Helmholtz equation. In this situation, the contribution [24] deals with the optimal repartition of an absorbing material (accounted for by Robin-like boundary conditions) on the walls of a room in order to minimize the sound pressure.

From the mathematical point of view, the above problems are of unequal difficulty: the calculation of the shape derivative $J^{\prime}(\Omega)$ of the optimized criterion $J(\Omega)$ (or that of the constraint functions) in the framework of Hadamard's method depends very much on the regularity of the physical state $u_{\Omega}$ of the problem in the neighborhood of the optimized transition region between zones bearing different types of boundary conditions. In some of the above situations, $u_{\Omega}$ is 'smooth enough' near these transitions, and the calculation of $J^{\prime}(\Omega)$ is achieved by classical means. On the contrary, the situation become much more difficult to analyze when $u_{\Omega}$ happens to be 'weakly singular' near these transitions; indeed, the calculation of the shape derivative of $J(\Omega)$ requires a precise knowledge of this singular behavior of $u_{\Omega}$. The resulting formula is also quite difficult to handle in algorithmic practice, since it brings into play quantities which somehow measure this singular behavior, that are difficult to evaluate from the numerical viewpoint. To the best of our knowledge, the first theoretical calculation of shape derivatives in a context where regions bearing different types of boundary conditions are optimized - dealing with the difficulty of a 'weakly singular' state $u_{\Omega}$ - dates back to [28]. Such calculations have also been considered in a series of articles [8, 47] by the so-called Generalized $J$-integral method. The authors of the latter references propose a mesh refinement procedure in order to achieve an accurate numerical calculation of the state function in the neighborhood of the transition between regions of $\partial \Omega$ with different boundary conditions.

Our purpose in this article is to study such shape optimization problems in which the regions of the boundary of the optimized shape $\Omega$ bearing different types of boundary conditions are subject to optimization. Most of the theoretical analysis unfolds in a model two-dimensional situation where a functional $J(\Omega)$ of the shape $\Omega$ is minimized; $J(\Omega)$ depends on $\Omega$ via a state $u_{\Omega}$ which is the solution to a Laplace equation with mixed boundary conditions: the boundary $\partial \Omega$ is divided into three regions $\Gamma_{D}, \Gamma_{N}$ and $\Gamma$, and $u_{\Omega}$ satisfies homogeneous Dirichlet boundary conditions on $\Gamma_{D}$, inhomogeneous Neumann boundary conditions on $\Gamma_{N}$, and homogeneous Neumann boundary conditions on $\Gamma$; see (2.2) below. At first, we rigorously calculate the derivative $J^{\prime}(\Omega)$ of $J(\Omega)$ with respect to variations of the shape $\Omega$ in both situations where the transitions $\Sigma_{N}=\bar{\Gamma} \cap \overline{\Gamma_{N}}$ and $\Sigma_{D}=\bar{\Gamma} \cap \overline{\Gamma_{D}}$ are also subject to optimization. In the former case, the shape derivative turns out to have a classical structure, and it lends itself to a fairly simple treatment in numerical algorithms. On the contrary, in the latter context, the state $u_{\Omega}$ is weakly singular near $\Sigma_{D}$, which makes the formula for $J^{\prime}(\Omega)$ uneasy to handle in practice. To circumvent this drawback, our second contribution is to propose an approximation method for the considered state problem (2.2), and thereby for the resulting shape optimization problem: elaborating on the idea developped in [24] and on our previous work [4], the considered 'exact' Laplace equation with mixed boundary conditions is replaced with an approximate counterpart, parametrized by a 'small' parameter $\varepsilon$, where Robin boundary conditions with $\varepsilon$-varying coefficients are imposed on the whole boundary $\partial \Omega$ : the 'sharp' transition $\Sigma_{D}$ between regions equipped with homogeneous Dirichlet and Neumann boundary conditions is thus 'smeared' into a zone with thickness $\varepsilon$; the shape optimization problem of the counterpart $J_{\varepsilon}(\Omega)$ of $J(\Omega)$ where $u_{\Omega}$ is replaced with the approximate state $u_{\Omega, \varepsilon}$ lends itself to an easier mathematical analysis: in particular, the approximate shape derivative $J_{\varepsilon}^{\prime}(\Omega)$ does not involve any singular component, and it is therefore much easier to use in practice. We then turn to prove the consistency of this approach: namely, the approximate objective function $J_{\varepsilon}(\Omega)$ and its shape derivative $J_{\varepsilon}^{\prime}(\Omega)$ converge to their exact counterparts $J(\Omega)$ and $J^{\prime}(\Omega)$ when the smoothing parameter $\varepsilon$ vanishes.

Let us emphasize that although this approximation method is rigorously justified only in a model situation, it is easy to generalize (and thus to use in a formal way) to many different, more involved situations, such as that of the linearized elasticity system (in two or three space dimensions), and we discuss several numerical examples in this spirit.

The remainder of this article is organized as follows. In Section 2, we present the considered model situation, involving the solution $u_{\Omega}$ to a Laplace equation with mixed boundary conditions, and we recall some 'classical', albeit technical material about the regularity of the state function $u_{\Omega}$ in the neighborhood of the transition between regions associated to different types of boundary conditions. In Section 3, we carefully calculate the shape derivative of a generic objective functional $J(\Omega)$ of the domain involving $u_{\Omega}$ in both contexts where the transitions $\Sigma_{N}$ and $\Sigma_{D}$ between, respectively, inhomogeneous Neumann - homogeneous Neumann and homogeneous Dirichlet - homogeneous Neumann boundary conditions are also subject to 
optimization. In Section 4, we focus on the second situation, which turns out to be especially intricate. We propose an approximation of the considered state problem which makes it much easier to analyze from the theoretical viewpoint, and supplies an approximate version $J_{\varepsilon}(\Omega)$ of $J(\Omega)$ which is quite convenient to use in numerical practice (in particular, its shape derivative is easier to evaluate); we then prove the consistency of this approximation process. The efficiency of the methods proposed in this article is illustrated in Section 5: model, analytical test-cases are investigated as well as more realistic applications such as optimization examples of $3 \mathrm{~d}$ elastic structures. This article ends with the technical Appendix A in which some useful facts about tangential calculus are recalled.

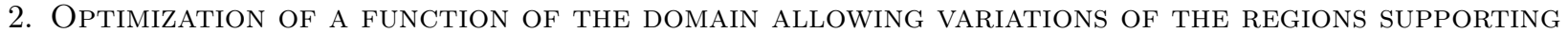 BOUNDARY CONDITIONS}

In this section, we present the model problem under scrutiny in most of the article, which concentrates the main difficulties we plan to address in a simplified setting, and lends itself to a rather complete mathematical analysis.

\subsection{Presentation of the model physical problem and notations}

Let $\Omega \subset \mathbb{R}^{d}$ be a smooth bounded domain ( $d=2$ or 3 in applications), whose boundary $\partial \Omega$ is divided into three disjoint, complementary open regions $\Gamma_{D} \neq \emptyset, \Gamma_{N}$ and $\Gamma$ :

$$
\partial \Omega=\overline{\Gamma_{D}} \cup \overline{\Gamma_{N}} \cup \bar{\Gamma}
$$

We denote by $u_{\Omega}$ the unique solution in the space

$$
H_{\Gamma_{D}}^{1}(\Omega):=\left\{u \in H^{1}(\Omega), u=0 \text { on } \Gamma_{D}\right\}
$$

to the following mixed boundary value problem:

$$
\left\{\begin{array}{cl}
-\Delta u_{\Omega}=f & \text { in } \Omega, \\
u_{\Omega}=0 & \text { on } \Gamma_{D}, \\
\frac{\partial u_{\Omega}}{\partial n}=g & \text { on } \Gamma_{N}, \\
\frac{\partial u_{\Omega}}{\partial n}=0 & \text { on } \Gamma,
\end{array}\right.
$$

where $n$ is the unit normal vector to $\partial \Omega$, pointing outward $\Omega$, and the source term $f$ and boundary flux $g$ are supposed to be regular enough, say $f \in L^{2}\left(\mathbb{R}^{d}\right), g \in H^{1}\left(\mathbb{R}^{d}\right)$. Note that $g$ may vanish on some subset of $\Gamma_{N}$, so that the inclusion $\Gamma \subset\left\{x \in \partial \Omega, \frac{\partial u_{\Omega}}{\partial n}=0\right\}$ may be strict.

In this context, we denote by $\Sigma_{D}=\overline{\Gamma_{D}} \cap \bar{\Gamma} \subset \partial \Omega$ (resp. $\Sigma_{N}=\overline{\Gamma_{N}} \cap \bar{\Gamma} \subset \partial \Omega$ ) the boundary between the region $\Gamma_{D} \subset \partial \Omega$ bearing homogeneous Dirichlet boundary conditions (resp. the region $\Gamma_{N} \subset \partial \Omega$ bearing inhomogeneous Neumann boundary conditions) and that $\Gamma$ endowed with homogeneous Neumann boundary conditions; for simplicity, we assume that $\overline{\Gamma_{D}} \cap \overline{\Gamma_{N}}=\emptyset$. The sets $\Sigma_{D}$ and $\Sigma_{N}$ are both assumed to be smooth, codimension 1 submanifolds of $\partial \Omega$ : in particular, they amount to collections of isolated points in the case $d=2$, and to sets of smooth closed curves drawn on $\partial \Omega$ if $d=3$. We denote by $n_{\Sigma_{D}}: \Sigma_{D} \rightarrow \mathbb{R}^{d}$ (resp. $n_{\Sigma_{N}}: \Sigma_{N} \rightarrow \mathbb{R}^{d}$ ) the unit normal vector to $\Sigma_{D}\left(\right.$ resp. $\left.\Sigma_{N}\right)$ pointing outward $\Gamma_{D}\left(\right.$ resp. $\left.\Gamma_{N}\right)$, inside the tangent plane to $\partial \Omega$; see Fig. 1 (left) for an illustration of these definitions.

On several occurrences, the rigorous mathematical analysis of this model will be greatly simplified under further simplifying assumptions; in particular, in some duly specified situations, we shall proceed under the following hypotheses, in the situation where $d=2$ :

The region $\overline{\Gamma_{D}} \cap \bar{\Gamma}$ consists of only two points $\overline{\Gamma_{D}} \cap \bar{\Gamma}=\left\{s_{0}, s_{1}\right\}$,

and

$$
\text { the domain } \Omega \subset \mathbb{R}^{2} \text { is locally flat around } s_{0}, s_{1} \text {; }
$$

see Fig. 1 (right). In this last case, letting $n=\left(n_{1}, n_{2}\right)$, we shall denote by $\tau:=\left(n_{2},-n_{1}\right)$ the unit tangent vector to $\partial \Omega$, oriented so that $(\tau, n)$ is a direct orthonormal frame of the plane. 

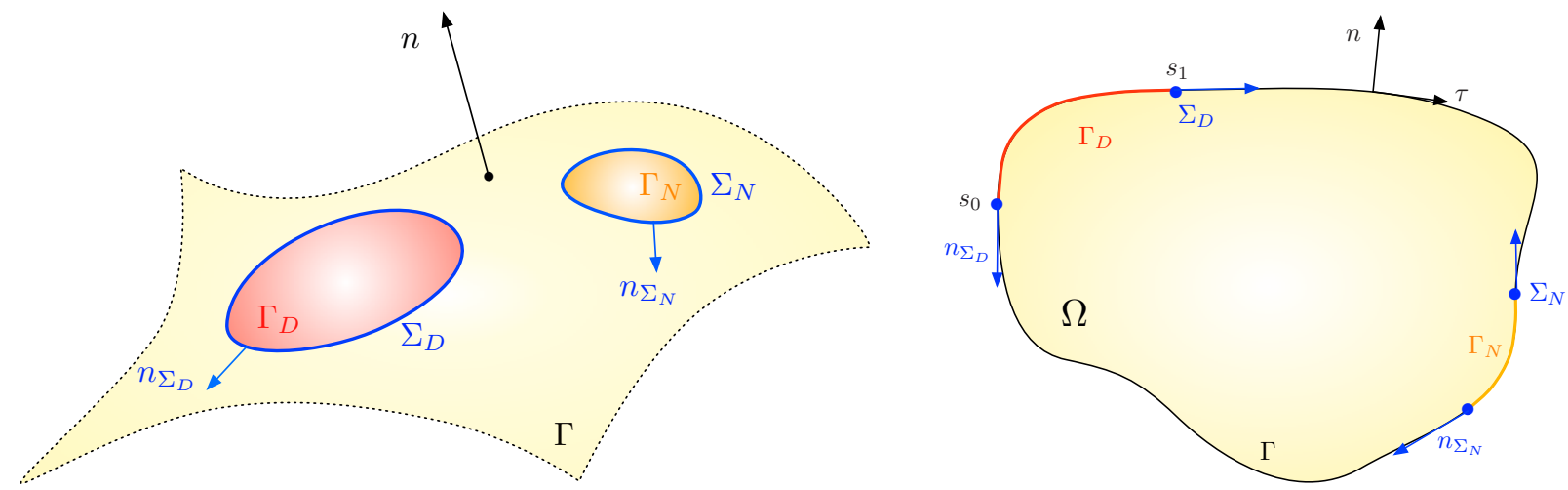

Figure 1. (Left) Sketch of the considered setting in Section 2.1; (right) the simplified situation where Assumption (2.3) is fulfilled.

\subsection{The shape optimization problem}

In the setting of Section 2.1, we consider the following shape optimization problem:

$$
\inf _{\Omega \in \mathcal{U}_{\mathrm{ad}}} J(\Omega)
$$

featuring an objective criterion $J(\Omega)$ of the form

$$
J(\Omega)=\int_{\Omega} j\left(u_{\Omega}\right) d x
$$

where $j: \mathbb{R} \rightarrow \mathbb{R}$ is a smooth function satisfying appropriate growth conditions: there exists a constant $C>0$ such that:

$$
\forall t \in \mathbb{R},|j(t)| \leq C\left(1+t^{2}\right), \quad\left|j^{\prime}(t)\right| \leq C(1+|t|), \text { and }\left|j^{\prime \prime}(t)\right| \leq C .
$$

In (2.4), $\mathcal{U}_{\text {ad }}$ is a set of smooth admissible shapes; in the following, two distinct shape optimization problems of this form are considered, implying different definitions of $\mathcal{U}_{\text {ad }}$ :

- On the one hand, the transition $\Sigma_{D}$ between homogeneous Dirichlet and homogeneous Neumann boundary conditions is subject to optimization, and the region $\Gamma_{N}$ bearing inhomogeneous Neumann boundary conditions is fixed. Then, $\mathcal{U}_{\text {ad }}$ corresponds to the set:

$$
\mathcal{U}_{\mathrm{DN}}:=\left\{\Omega \subset \mathbb{R}^{d} \text { is bounded and of class } \mathcal{C}^{2}, \Gamma_{N} \subset \partial \Omega\right\} .
$$

- On the other hand, when the region $\Sigma_{N}$ between $\Gamma$ and $\Gamma_{N}$ is optimized (while the region $\Gamma_{D} \subset \partial \Omega$ is fixed), $\mathcal{U}_{\text {ad }}$ reads:

$$
\mathcal{U}_{\mathrm{NN}}:=\left\{\Omega \subset \mathbb{R}^{d} \text { is bounded and of class } \mathcal{C}^{2}, \Gamma_{D} \subset \partial \Omega\right\} .
$$

Notice that problem (2.4) is not guaranteed to have a solution; nevertheless, we assume in the following that it is the case or that, at least, local minima exist. Moreover, observe that the objective function $J(\Omega)$ featured in (2.5) and the solution $u_{\Omega}$ to (2.2) depend on the particular subdivision (2.1) of the boundary $\partial \Omega$ into $\Gamma_{D}, \Gamma_{N}$ and $\Gamma$; with some little abuse and so as to keep notations simple insofar as possible, this dependence is not made explicit in the formulation of our shape optimization problem.

Most practical optimization algorithms for solving problems of the form (2.4) rely on the derivative of $J(\Omega)$, which calls for a notion of differentiation with respect to the domain. Several ways are available to achieve this, and in the present work, we rely on Hadamard's boundary variation method; see e.g. [6, 33, 46, 53]. In a nutshell, variations of a domain $\Omega$ are considered under the form:

$$
\Omega_{\theta}:=(\operatorname{Id}+\theta)(\Omega), \quad \theta \in W^{1, \infty}\left(\mathbb{R}^{d}, \mathbb{R}^{d}\right),\|\theta\|_{W^{1, \infty}\left(\mathbb{R}^{d}, \mathbb{R}^{d}\right)}<1 .
$$

Accordingly, a function of the domain $F(\Omega)$ is said to be shape differentiable at $\Omega$ if the underlying mapping $\theta \mapsto F\left(\Omega_{\theta}\right)$, from $W^{1, \infty}\left(\mathbb{R}^{d}, \mathbb{R}^{d}\right)$ into $\mathbb{R}$, is Fréchet differentiable at $\theta=0$. The following expansion then 
holds in the neighborhood of $\theta=0$ :

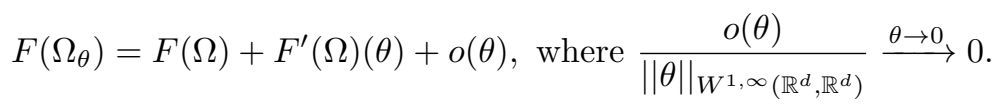

In practice, so that variations of an admissible shape stay admissible, the considered deformations $\theta$ are confined to a subset $\Theta_{\mathrm{ad}} \subset W^{1, \infty}\left(\mathbb{R}^{d}, \mathbb{R}^{d}\right)$ of admissible deformations. In the present article, $\Theta_{\mathrm{ad}}$ stands for one of the sets $\Theta_{\mathrm{DN}}$ or $\Theta_{\mathrm{NN}}$ defined by:

$$
\Theta_{\mathrm{DN}}:=\left\{\theta \in \mathcal{C}^{2}\left(\mathbb{R}^{d}, \mathbb{R}^{d}\right) \cap W^{1, \infty}\left(\mathbb{R}^{d}, \mathbb{R}^{d}\right), \theta=0 \text { on } \Gamma_{N}\right\},
$$

and

$$
\Theta_{\mathrm{NN}}:=\left\{\theta \in \mathcal{C}^{2}\left(\mathbb{R}^{d}, \mathbb{R}^{d}\right) \cap W^{1, \infty}\left(\mathbb{R}^{d}, \mathbb{R}^{d}\right), \theta=0 \text { on } \Gamma_{D}\right\},
$$

both being equipped with the natural norm, when the considered set of admissible shapes is $\mathcal{U}_{\mathrm{DN}}$ or $\mathcal{U}_{\mathrm{NN}}$, respectively.

Remark 2.1. In practice, it often turns out that the expression of the shape derivative $J^{\prime}(\Omega)(\theta)$ of the considered objective function does not readily allow to identify a descent direction for $J(\Omega)$, that is, a deformation $\theta \in \Theta_{\text {ad }}$ such that $J^{\prime}(\Omega)(\theta)<0$. To remedy this difficulty, we rely on the 'change of inner products' strategy presented, e.g. in [14, 21]: in a nutshell, a suitable Hilbert space $\mathcal{H} \subset \Theta_{\mathrm{ad}}$ is selected, with inner product $a(\cdot, \cdot)$, and the gradient associated to the derivative $J^{\prime}(\Omega)(\theta)$ via this inner product is calculated as the solution to the following problem:

$$
\text { Search for } V \in \mathcal{H} \text { s.t. } \forall \xi \in \mathcal{H}, a(V, \xi)=J^{\prime}(\Omega)(\xi) .
$$

It then follows immediately from (2.6) that $\theta=-V$ is a descent direction for $J(\Omega)$. A popular strategy consists in taking $\mathcal{H}=H^{1}(D)^{d}$ (where $D$ is a large computational domain), equipped with the inner product

$$
a(\theta, \xi)=\alpha^{2} \int_{D} \nabla \theta: \nabla \xi d x+\int_{D} \theta \cdot \xi d x,
$$

where the parameter $\alpha$ is chosen of the order of the mesh size; the resolution of (2.9) then boils down to that of a Laplace-like equation. Note that this choice is only formal (since $\mathcal{H}$ may not be contained in $\Theta_{\mathrm{ad}}$ ) but it turns out to work well in practice.

Remark 2.2. In principle, the shape optimization problem (2.4) could be supplemented with $p$ constraints of the form

$$
C_{i}(\Omega) \leq 0, i=1, \ldots, p
$$

since this does not add anything to the points we intend to highlight in this article, but only increases the difficulty of the numerical treatment of (2.4), we ignore these issues.

\subsection{A brief account of the regularity of $u_{\Omega}$}

As is well-known in the field of elliptic boundary value problems (see e.g. [2], or [13] §9.6 for a comprehensive introduction), when the featured data $f, g$ (and $\Omega$ itself) are smooth enough, the solution $u_{\Omega}$ to (2.2) is more regular than a mere element in $H^{1}(\Omega)$, as predicted by the classical Lax-Milgram variational theory. For instance, in the 'simple' case where (2.2) only brings into play homogeneous Dirichlet boundary conditions (i.e. $\Gamma$ and $\Gamma_{N}$ are empty), the classical 'shift theorem' states that, provided $f$ belongs to $H^{m}(\Omega)$ for some $m \geq 0$ (and $\Omega$ is smooth), $u_{\Omega}$ belongs to $H^{m+2}(\Omega)$.

In the situations at stake in the present article, such as (2.2), things are a little more subtle. The assumptions that $f \in L^{2}\left(\mathbb{R}^{d}\right)$ and $g \in H^{1}\left(\mathbb{R}^{d}\right)$ still guarantee that $u_{\Omega}$ has $H^{2}$ regularity in some open neighborhood of an arbitrary point $x_{0}$ which is either interior to $\Omega$, or which belongs to $\partial \Omega$ but is interior to one of the regions $\Gamma_{D}, \Gamma$ or $\Gamma_{N}$. On the contrary, $u_{\Omega}$ has limited regularity around those points $x_{0} \in \Sigma_{D}$ or $x_{0} \in \Sigma_{N}$ marking the transition between regions subjected to different types of boundary conditions. A whole mathematical theory exists in the literature, which is devoted to the precise study of the 'weakly singular' behavior of $u_{\Omega}$ in these regions; we refer for instance to the monographs [20, 30, 37].

In this section, we first recall briefly some classical material about functional spaces in Section 2.3.1, before summarizing in Section 2.3.2 the needed results about the regularity of the solution $u_{\Omega}$ to (2.2) for our purposes. 


\subsubsection{Some functional spaces}

Let $\Omega$ be a smooth bounded domain in $\mathbb{R}^{d}$. For $s>0$ and $1<p<\infty$, let us introduce:

- The usual Sobolev space $W^{s, p}(\Omega)$ is defined by, when $s=m$ is an integer:

$$
W^{m, p}(\Omega)=\left\{u \in L^{p}(\Omega), \partial^{\alpha} u \in L^{p}(\Omega) \text { for } \alpha \in \mathbb{N}^{d},|\alpha| \leq m\right\},
$$

and when $s=m+\sigma$ with $m \in \mathbb{N}$ and $\sigma \in(0,1)$,

$$
W^{s, p}(\Omega)=\left\{u \in W^{m, p}(\Omega), \quad \int_{\Omega} \int_{\Omega} \frac{\left|\partial^{\alpha} u(x)-\partial^{\alpha} u(y)\right|^{p}}{|x-y|^{d+p \sigma}} d y d x<\infty \text { for all }|\alpha|=m\right\} .
$$

Both sets are equipped with the natural norms. Let us recall that the exact same definitions hold when the bounded domain $\Omega$ is replaced by the whole space $\mathbb{R}^{d}$.

- The subspace $\widetilde{W}^{s, p}(\Omega)$ of $W^{s, p}(\Omega)$ is that composed of functions whose extension $\widetilde{u}$ by 0 outside $\Omega$ belongs to $W^{s, p}\left(\mathbb{R}^{d}\right)$.

- The space $W_{0}^{s, p}(\Omega)$ is the closure of the set $\mathcal{C}_{c}^{\infty}(\Omega)$ of $\mathcal{C}^{\infty}$ functions with compact support in $\Omega$ in $W^{s, p}(\Omega)$.

As is customary, in the case $p=2$ we use the notations $H^{s}(\Omega), \widetilde{H}^{s}(\Omega)$ and $H_{0}^{s}(\Omega)$ for $W^{s, p}(\Omega), \widetilde{W}^{s, p}(\Omega)$ and $W_{0}^{s, p}(\Omega)$ respectively.

In spite of their tight relation, the two spaces $\widetilde{W}^{s, p}(\Omega)$ and $W_{0}^{s, p}(\Omega)$ may not coincide depending on the values of $s$ and $p$. On the one hand, for any $s>0$ and $1<p<\infty, W_{0}^{s, p}(\Omega) \subset \widetilde{W}^{s, p}(\Omega)$, but the converse inclusion may fail. In fact, the following characterization holds (see [30], Lemma 1.3.2.6 and Corollary 1.4.4.10):

$$
\widetilde{W}^{s, p}(\Omega)=\left\{u \in W_{0}^{m, p}(\Omega), \frac{1}{\rho^{\sigma}} \partial^{\alpha} u \in L^{p}(\Omega),|\alpha|=m\right\},
$$

where we have decomposed $s=m+\sigma$, with $m \in \mathbb{N}$ and $\sigma \in(0,1)$, and $\rho(x):=d(x, \partial \Omega)$ is the (unsigned) distance from $x$ to the boundary of $\Omega$. The space $\widetilde{W}^{s, p}(\Omega)$ is then endowed with the norm

$$
\|u\|_{\widetilde{W}^{s, p}(\Omega)}=\left(\|u\|_{W^{m, p}(\Omega)}^{p}+\sum_{|\alpha|=m} \int_{\Omega} \frac{1}{\rho^{p \sigma}}\left|\partial^{\alpha} u\right|^{p} d x\right)^{\frac{1}{p}},
$$

which is equivalent to the natural norm $u \mapsto\|\widetilde{u}\|_{W^{s, p}\left(\mathbb{R}^{d}\right)}$. Let us also note that:

$$
\widetilde{W}^{s, p}(\Omega)=W_{0}^{s, p}(\Omega) \text { when }\left(s-\frac{1}{p}\right) \text { is not an integer. }
$$

We eventually mention that the above definitions and results hold in the more general context where $\Omega$ is replaced by a smooth submanifold of $\mathbb{R}^{d}$, e.g. (a region of) the boundary of a bounded smooth domain of $\mathbb{R}^{d}$. For instance, in the setting of Section 2.1, we may consider the spaces $\widetilde{W}^{s, p}\left(\Gamma_{D}\right), \widetilde{W}^{s, p}\left(\Gamma_{N}\right)$, etc.

\subsubsection{Local structure of $u_{\Omega}$ near the transition $\Sigma_{D}$}

The classical variational theory for (2.2) (based on the Lax-Milgram theorem) features a solution $u_{\Omega}$ which naturally belongs to $H^{1}(\Omega)$. Moreover, assuming that the boundary $\partial \Omega$ is at least of class $\mathcal{C}^{2}$, and that $f \in L^{2}\left(\mathbb{R}^{d}\right), g \in H^{1}\left(\mathbb{R}^{d}\right)$, the 'classical' elliptic regularity theory ensures that $u_{\Omega}$ actually has $H^{2}$ regularity except perhaps near the transitions zones $\Sigma_{D}$ and $\Sigma_{N}$; see [2], and [13], §9.6. On the contrary, $u_{\Omega}$ fails to enjoy $H^{2}$ regularity in the vicinity of $\Sigma_{D}$ or $\Sigma_{N}$, where the boundary conditions it fulfills change types.

The precise behavior of $u_{\Omega}$ near $\Sigma_{D}$ will be of utmost interest for our purpose; it is exemplified by the following theorem, which takes place under Assumption (2.3) (see [30], Chap. 4 and notably Th. 4.4.3.7):

Proposition 2.1. For any point $x_{0} \in \Omega$ or $x_{0} \in \partial \Omega \backslash\left(\Sigma_{D} \cup \Sigma_{N}\right)$, there exists an open neighborhood $W$ of $x_{0}$ in $\mathbb{R}^{2}$ such that $u_{\Omega}$ belongs to $H^{2}(\Omega \cap W)$. Furthermore, for either $i=0$ or $i=1$, there exists an open neighborhood $V$ of $s_{i}$ with the following property: introducing the polar coordinates $(r, \nu)$ at $s_{i}$, assuming without 
loss of generality that $s_{i}=0, \Omega \cap V=\left\{x \in V\right.$, s.t. $\left.x_{2}>0\right\}$, and $\Gamma_{D} \cap V=\left\{x \in V\right.$, s.t. $\left.x_{2}=0, x_{1}<0\right\}$, there exist a function $u_{r}^{i} \in H^{2}(V)$, and a constant $c^{i} \in \mathbb{R}$ such that:

$$
u_{\Omega}=u_{r}^{i}+c^{i} S^{i} \text { on } V \text {, where } S^{i}(r, \nu)=r^{\frac{1}{2}} \cos \left(\frac{\nu}{2}\right) .
$$

The function $S^{i}$ is sometimes said to be weakly singular, in the sense that it belongs to $H^{1}(V)$, but not to $H^{2}(V)$. More precisely, invoking Theorem 1.4.5.3 in [30] to estimate the Sobolev regularity of functions of the form $r^{\alpha} \varphi(\nu)$, one proves that, for every $0 \leq s<\frac{3}{2}$, $u_{\Omega} \in H^{s}(V)$, with:

$$
\left\|u_{\Omega}\right\|_{H^{s}(V)} \leq C_{s}\|f\|_{L^{2}\left(\mathbb{R}^{d}\right)} .
$$

In the language of Section 2.3.1, it follows in particular that $u_{\Omega} \in \widetilde{H}^{\frac{1}{2}}\left(\Gamma \cup \Gamma_{N}\right)$, while $\frac{\partial u_{\Omega}}{\partial n} \in\left(\widetilde{H}^{\frac{1}{2}}\left(\Gamma \cup \Gamma_{N}\right)\right)^{*}$.

Remark 2.3. Higher-order versions of the expansion (2.12) are available. Actually, for any integer $m \geq 2$, if $f \in H^{m-2}\left(\mathbb{R}^{d}\right)$, the following decomposition holds in a neighborhood $V$ of $s_{i}$ (see [30], Th. 5.1.3.5):

$$
u_{\Omega}=u_{r, m}^{i}+\sum_{k=1}^{m-1} c_{k}^{i} S_{k}^{i} \text {, where } u_{r, m}^{i} \in H^{m}(V) \text { and } S_{k}^{i}(r, \nu)=r^{k-\frac{1}{2}} \cos \left(\left(k-\frac{1}{2}\right) \nu\right) .
$$

Remark 2.4. Still in the two-dimensional context, when the boundary $\partial \Omega$ is not flat in the vicinity of $\Sigma_{D}$, an expansion of the form (2.12) still holds: the weakly singular function $S^{i}$ shows the same dependence $r^{\frac{1}{2}}$ with respect to $r$, but its dependence with respect to $\nu$ is no longer explicit. Nevertheless, there still holds that $u_{\Omega} \in H^{s}(V)$ with an estimate of the form (2.13), where $V$ is an open neigborhood of $\Sigma_{D}$ in $\Omega$ and $0 \leq s<\frac{3}{2}$ is arbitrary; see [30], Chap. 5 or [18].

\section{Shape Derivatives of the functional $J(\Omega)$}

In this section, we rigorously calculate the shape derivative of the objective function (2.4); we start in Section 3.1 with the case where the transition region $\Sigma_{N}$ is optimized and $\Sigma_{D}$ is fixed (i.e. the sets of admissible shapes and admissible deformations are $\mathcal{U}_{\mathrm{NN}}$ and $\Theta_{\mathrm{NN}}$ respectively), before turning in Section 3.2 to the more difficult case where $\Sigma_{D}$ is optimized and $\Sigma_{N}$ is not - i.e. $\mathcal{U}_{\mathrm{ad}}=\mathcal{U}_{\mathrm{DN}}$ and $\Theta_{\mathrm{ad}}=\Theta_{\mathrm{DN}}-$ under the simplifying assumption (2.3).

\subsection{Calculation of the shape derivative of $J(\Omega)$ when only the homogeneous Neumann - inho- mogeneous Neumann transition is optimized}

Our main result in this section is the following.

Proposition 3.1. The functional $J(\Omega)$ defined by $(2.5)$ is shape differentiable over the admissible set $\mathcal{U}_{\mathrm{NN}}$; its shape derivative reads (volumetric form):

$$
\begin{aligned}
& \forall \theta \in \Theta_{\mathrm{NN}}, \quad J^{\prime}(\Omega)(\theta)=\int_{\partial \Omega}\left(j\left(u_{\Omega}\right)-f p_{\Omega}\right) \theta \cdot n d s-\int_{\Omega} j^{\prime}\left(u_{\Omega}\right) \nabla u_{\Omega} \cdot \theta d x \\
& \quad+\int_{\Omega}\left(\operatorname{div} \theta \mathrm{I}-\nabla \theta-\nabla \theta^{T}\right) \nabla u_{\Omega} \cdot \nabla p_{\Omega} d x+\int_{\Omega} f \nabla p_{\Omega} \cdot \theta d x-\int_{\Gamma_{N}}\left(\left(\operatorname{div}_{\partial \Omega} \theta\right) g+\nabla g \cdot \theta\right) p_{\Omega} d s,
\end{aligned}
$$

where $\operatorname{div}_{\partial \Omega}$ stands for the tangential divergence on $\partial \Omega$ (see Appendix A), and the adjoint state $p_{\Omega}$ is the unique solution in $H_{\Gamma_{D}}^{1}(\Omega)$ to the system:

$$
\left\{\begin{array}{cl}
-\Delta p_{\Omega}=-j^{\prime}\left(u_{\Omega}\right) & \text { in } \Omega \\
p_{\Omega}=0 & \text { on } \Gamma_{D} \\
\frac{\partial p_{\Omega}}{\partial n}=0 & \text { on } \Gamma_{N} \cup \Gamma
\end{array}\right.
$$

The above shape derivative has the alternative, surfacic form:

$$
\begin{aligned}
\forall \theta \in \Theta_{\mathrm{NN}}, J^{\prime}(\Omega)(\theta)=\int_{\Gamma \cup \Gamma_{N}} j\left(u_{\Omega}\right) \theta \cdot n d s+ & \int_{\Gamma \cup \Gamma_{N}} \nabla u_{\Omega} \cdot \nabla p_{\Omega} \theta \cdot n d s-\int_{\Gamma \cup \Gamma_{N}} f p_{\Omega} \theta \cdot n d s \\
& -\int_{\Gamma_{N}}\left(\frac{\partial g}{\partial n}+\kappa g\right) p_{\Omega} \theta \cdot n d s-\int_{\Sigma_{N}} g p_{\Omega} \theta \cdot n_{\Sigma_{N}} d \ell .
\end{aligned}
$$


Remark 3.1. One comment is in order about the precise meaning of (3.3), and notably that of the term

$$
\int_{\Gamma \cup \Gamma_{N}} \nabla u_{\Omega} \cdot \nabla p_{\Omega} \theta \cdot n d s
$$

featured in there. The function $u_{\Omega}$ belongs to the space

$$
E\left(\Delta, L^{2}(\Omega)\right):=\left\{u \in H^{1}(\Omega), \Delta u \in L^{2}(\Omega)\right\},
$$

and as such, it has a normal trace $\frac{\partial u_{\Omega}}{\partial n} \in H^{-1 / 2}(\partial \Omega)$, which is defined by the Green's formula:

$$
\forall w \in H^{1}(\Omega), \quad \int_{\partial \Omega} \frac{\partial u_{\Omega}}{\partial n} w d s:=\int_{\Omega} \Delta u_{\Omega} w d x+\int_{\Omega} \nabla u_{\Omega} \cdot \nabla w d x ;
$$

see [30], Th. 1.5.3.10 for more details about this point. Also, since $u_{\Omega} \in H^{1 / 2}(\partial \Omega)$, the tangential derivative $\frac{\partial u_{\Omega}}{\partial \tau}$ naturally belongs to the dual space $H^{-1 / 2}(\partial \Omega)$. On the other hand, the function $p_{\Omega}$ enjoys $H^{2}$ regularity on account of elliptic regularity (see Section 2.3), except perhaps near $\Sigma_{D}$ where it has a weak singularity of the form (2.12). Since deformations $\theta \in \Theta_{\mathrm{NN}}$ are smooth and vanish identically on $\Gamma_{D}$, the product $\left(\nabla p_{\Omega}\right) \theta \cdot n$ has a trace in $H^{1 / 2}(\partial \Omega)$, and so the integral (3.4) is well-defined as a duality product.

Proof. The proof is an application of fairly classical techniques in shape optimization, except perhaps on one point regarding the regularity of $u_{\Omega}$ and $p_{\Omega}$. Since variations of this argument are used in the following, we present a sketch of it for the reader's convenience. The proof is decomposed into two steps.

Step 1: Proof of the shape differentiability of $J(\Omega)$ and derivation of (3.1).

This step amounts to the analysis of the differentiability of the mapping $\theta \mapsto J\left(\Omega_{\theta}\right)$ from $\Theta_{\mathrm{NN}}$ into $\mathbb{R}$, which features the solution $u_{\Omega_{\theta}}$ to the version of (2.2) posed on $\Omega_{\theta}$. The main idea consists in recasting the latter problem as a boundary-value problem on $\Omega$ for the transported function $\overline{u_{\theta}}:=u_{\Omega_{\theta}} \circ(\operatorname{Id}+\theta) \in H^{1}(\Omega)$; thence, the implicit function theorem allows to calculate the derivative of the mapping $\theta \mapsto \overline{u_{\theta}}$; see [33, 46].

For $\theta \in \Theta_{\mathrm{NN}}$ with norm $\|\theta\|_{\Theta_{\mathrm{NN}}}<1$, the function $u_{\Omega_{\theta}}$ is the unique solution in $H_{\Gamma_{D}}^{1}\left(\Omega_{\theta}\right)$ to the following variational problem:

$$
\forall v \in H_{\Gamma_{D}}^{1}\left(\Omega_{\theta}\right), \int_{\Omega_{\theta}} \nabla u_{\Omega_{\theta}} \cdot \nabla v d x=\int_{\Omega_{\theta}} f v d x+\int_{\left(\Gamma_{N}\right)_{\theta}} g v d s
$$

Using test functions of the form $v \circ(\operatorname{Id}+\theta)^{-1}, v \in H_{\Gamma_{D}}^{1}(\Omega)$, a change of variables yields the following variational formulation for $\overline{u_{\theta}} \in H_{\Gamma_{D}}^{1}(\Omega)$ :

$\forall v \in H_{\Gamma_{D}}^{1}(\Omega), \int_{\Omega} A(\theta) \nabla \overline{u_{\theta}} \cdot \nabla v d x=\int_{\Omega}|\operatorname{det}(\mathrm{I}+\nabla \theta)| f \circ(\mathrm{Id}+\theta) v d x+\int_{\Gamma_{N}}|\operatorname{com}(\mathrm{I}+\nabla \theta) n| g \circ(\mathrm{Id}+\theta) v d s$,

where $A(\theta)$ is the $d \times d$ matrix $A(\theta)=|\operatorname{det}(\mathrm{I}+\nabla \theta)|(\mathrm{I}+\nabla \theta)^{-1}(\mathrm{I}+\nabla \theta)^{-T}$ and $\operatorname{com}(M)$ stands for the cofactor matrix of a $d \times d$ matrix $M$. Now introducing the mapping $\mathcal{F}: \Theta_{\text {ad }} \times H_{\Gamma_{D}}^{1}(\Omega) \rightarrow\left(H_{\Gamma_{D}}^{1}(\Omega)\right)^{*}$ defined by:

$$
\begin{aligned}
\forall v \in H_{\Gamma_{D}}^{1}(\Omega), \mathcal{F}(\theta, u)(v)=\int_{\Omega} A(\theta) \nabla u \cdot \nabla v d x-\int_{\Omega}|\operatorname{det}(\mathrm{I}+\nabla \theta)| f \circ(\mathrm{Id}+\theta) v d x \\
-\int_{\Gamma_{N}}|\operatorname{com}(\mathrm{I}+\nabla \theta) n| g \circ(\mathrm{Id}+\theta) v d s,
\end{aligned}
$$

it follows that for 'small' $\theta \in \Theta_{\mathrm{NN}}, \bar{u}_{\theta}$ is the unique solution of the equation $\mathcal{F}\left(\theta, \bar{u}_{\theta}\right)=0$. Then, the implicit function theorem (see e.g. [38], Chap. 1, Th. 5.9) together with classical calculations imply that the mapping $\theta \mapsto \overline{u_{\theta}}$ is Fréchet differentiable from a neighborhood of 0 in $\Theta_{\mathrm{NN}}$ into $H_{\Gamma_{D}}^{1}(\Omega)$, and that its derivative $\theta \mapsto u_{\Omega}(\theta)$ at $\theta=0$ - the so-called 'Lagrangian derivative' of the mapping $\Omega \mapsto u_{\Omega}$ - is the unique solution to the following variational problem:

$$
\begin{aligned}
\forall v \in H_{\Gamma_{D}}^{1}(\Omega), \int_{\Omega} \nabla u_{\Omega}(\theta) \cdot \nabla v d x=-\int_{\Omega}(\operatorname{div} \theta \mathrm{I} & \left.-\nabla \theta-\nabla \theta^{T}\right) \nabla u_{\Omega} \cdot \nabla v d x \\
& +\int_{\Omega} \operatorname{div}(f \theta) v d x+\int_{\Gamma_{N}}\left(\left(\operatorname{div}_{\partial \Omega} \theta\right) g+\nabla g \cdot \theta\right) v d s .
\end{aligned}
$$


On the other hand, performing the same change of variables in the definition of $J(\Omega)$ yields:

$$
J\left(\Omega_{\theta}\right)=\int_{\Omega}|\operatorname{det}(\mathrm{I}+\nabla \theta)| j\left(\overline{u_{\theta}}\right) d x,
$$

and so the mapping $\theta \mapsto J\left(\Omega_{\theta}\right)$ from $\Theta_{\mathrm{NN}}$ into $\mathbb{R}$ is Fréchet differentiable at $\theta=0$ with derivative:

$$
J^{\prime}(\Omega)(\theta)=\int_{\Omega}\left(\operatorname{div} \theta j\left(u_{\Omega}\right)+j^{\prime}\left(u_{\Omega}\right) u_{\Omega}^{\circ}(\theta)\right) d x .
$$

The material derivative $u_{\Omega}^{\circ}(\theta)$ can now be eliminated from (3.8) thanks to the introduction of the adjoint state $p_{\Omega}$, solution to (3.2). Indeed, the variational formulation of $p_{\Omega}$ reads:

$$
\forall v \in H_{\Gamma_{D}}^{1}(\Omega), \int_{\Omega} \nabla p_{\Omega} \cdot \nabla v d x=-\int_{\Omega} j^{\prime}\left(u_{\Omega}\right) v d x
$$

Hence, combining (3.8) with (3.9) yields:

$$
\begin{aligned}
J^{\prime}(\Omega)(\theta)= & \int_{\Omega}(\operatorname{div} \theta) j\left(u_{\Omega}\right) d x-\int_{\Omega} \nabla p_{\Omega} \cdot \nabla u_{\Omega}^{\circ}(\theta) d x, \\
= & \int_{\Omega}(\operatorname{div} \theta) j\left(u_{\Omega}\right) d x+\int_{\Omega}\left((\operatorname{div} \theta) \mathrm{I}-\nabla \theta-\nabla \theta^{T}\right) \nabla u_{\Omega} \cdot \nabla p_{\Omega} d x-\int_{\Omega} \operatorname{div}(f \theta) p_{\Omega} d x \\
& -\int_{\Gamma_{N}}\left(\left(\operatorname{div}_{\partial \Omega} \theta\right) g+\nabla g \cdot \theta\right) p_{\Omega} d s, \\
= & \int_{\Omega} \operatorname{div}\left(j\left(u_{\Omega}\right) \theta\right) d x-\int_{\Omega} j^{\prime}\left(u_{\Omega}\right) \nabla u_{\Omega} \cdot \theta d x+\int_{\Omega}\left((\operatorname{div} \theta) \mathrm{I}-\nabla \theta-\nabla \theta^{T}\right) \nabla u_{\Omega} \cdot \nabla p_{\Omega} d x \\
& -\int_{\Omega} \operatorname{div}\left(f \theta p_{\Omega}\right) d x+\int_{\Omega} f \nabla p_{\Omega} \cdot \theta d x-\int_{\Gamma_{N}}((\operatorname{div} \partial \Omega) g+\nabla g \cdot \theta) p_{\Omega} d s,
\end{aligned}
$$

where we have used the variational formulation $(3.7)$ for $u_{\Omega}^{\circ}(\theta)$ to pass from the first line to the second one. This results in the desired expression (3.13). Note that at this point, we have not used the fact that either $u_{\Omega}$ or $p_{\Omega}$ is more regular than $H^{1}(\Omega)$.

Step 2: Derivation of the surface expression (3.3).

This expression is classically achieved from (3.1) using integration by parts; doing so requires a more careful attention to the regularity of $u_{\Omega}$ and $p_{\Omega}$.

Let us notice that the function $u_{\Omega}$ may not be much more regular than just $H^{1}$ in the neighborhood of the transition $\Sigma_{N}$. Actually, it belongs to the space $E\left(\Delta, L^{2}(\Omega)\right)$, defined by (3.5). The key point is that $p_{\Omega}$ is locally $H^{2}$ in the neighborhood of $\Sigma_{N}$, on account of the material in Section 2.3 (note that $\frac{\partial p_{\Omega}}{\partial n}=0$ on $\left.\Gamma \cup \Gamma_{N}\right)$. Relying on the following identity, which holds for smooth functions $v, w \in \mathcal{C}_{c}^{\infty}\left(\mathbb{R}^{d}\right)$,

$$
\begin{aligned}
\int_{\Omega}\left((\operatorname{div} \theta) \mathrm{I}-\nabla \theta-\nabla \theta^{T}\right) \nabla v \cdot \nabla w d x= & \int_{\Gamma \cup \Gamma_{N}}\left((\nabla v \cdot \nabla w) \theta \cdot n-\frac{\partial v}{\partial n} \nabla w \cdot \theta-\frac{\partial w}{\partial n} \nabla v \cdot \theta\right) d s \\
& +\int_{\Omega}\left(-\nabla(\nabla v \cdot \nabla w)+\Delta v \nabla w+\Delta w \nabla v+\nabla^{2} w \nabla v+\nabla^{2} v \nabla w\right) \cdot \theta d x \\
= & \int_{\Gamma \cup \Gamma_{N}}\left((\nabla v \cdot \nabla w) \theta \cdot n-\frac{\partial v}{\partial n} \nabla w \cdot \theta-\frac{\partial w}{\partial n} \nabla v \cdot \theta\right) d s \\
& +\int_{\Omega}(\Delta v \nabla w+\Delta w \nabla v) \cdot \theta d x
\end{aligned}
$$

and using the density of $\mathcal{C}_{c}^{\infty}\left(\mathbb{R}^{d}\right)$ in $E\left(\Delta, L^{2}(\Omega)\right)$ and $H^{2}(\Omega)$ (see [30], Lemma 1.5.3.9), we obtain:

$$
\begin{array}{r}
\int_{\Omega}\left((\operatorname{div} \theta) \mathrm{I}-\nabla \theta-\nabla \theta^{T}\right) \nabla u_{\Omega} \cdot \nabla p_{\Omega} d x=\int_{\Gamma \cup \Gamma_{N}}\left(\left(\nabla u_{\Omega} \cdot \nabla p_{\Omega}\right) \theta \cdot n-\frac{\partial u_{\Omega}}{\partial n} \nabla p_{\Omega} \cdot \theta-\frac{\partial p_{\Omega}}{\partial n} \nabla u_{\Omega} \cdot \theta\right) d s \\
+\int_{\Omega}\left(\Delta u_{\Omega} \nabla p_{\Omega}+\Delta p_{\Omega} \nabla u_{\Omega}\right) \cdot \theta d x .
\end{array}
$$


Let us now work on the last integral in the right-hand side of (3.1); we obtain:

$$
\begin{aligned}
\left(\operatorname{div}_{\partial \Omega} \theta\right) g+\nabla g \cdot \theta & =\operatorname{div}_{\partial \Omega}(g \theta)+\frac{\partial g}{\partial n} \theta \cdot n, \\
& =\operatorname{div}_{\partial \Omega}(g(\theta-(\theta \cdot n) n))+\left(\frac{\partial g}{\partial n}+\kappa g\right) \theta \cdot n .
\end{aligned}
$$

Hence, an integration by parts on the region $\Gamma_{N}$ using Proposition A.1 yields:

$$
\int_{\Gamma_{N}}\left(\left(\operatorname{div}_{\partial \Omega} \theta\right) g+\nabla g \cdot \theta\right) p_{\Omega} d s=\int_{\Sigma_{N}} g p_{\Omega} \theta \cdot n_{\Sigma_{N}} d \ell+\int_{\Gamma_{N}}\left(\frac{\partial g}{\partial n}+\kappa g\right) p_{\Omega} \theta \cdot n d s .
$$

Combining (3.11) and (3.12) with the volumetric formula (3.1) and using the facts that $-\Delta u_{\Omega}=f$ and $-\Delta p_{\Omega}=-j^{\prime}\left(u_{\Omega}\right)$ in $\Omega$, we finally obtain the desired surface formula (3.3).

Remark 3.2. This calculation extends readily to the linearized elasticity case, to deal with NeumannNeumann transitions.

\subsection{Calculation of the shape derivative of $J(\Omega)$ when only the Dirichlet-homogeneous Neumann transition is optimized}

In this section, we investigate the shape differentiability of the functional $J(\Omega)$ defined in $(2.5)$ in the particular case where the boundary $\Sigma_{D}$ between the regions $\Gamma_{D}$ and $\Gamma$ of $\partial \Omega$ bearing homogeneous Dirichlet and homogeneous Neumann boundary conditions is also subject to optimization; in other terms, we suppose:

$$
\mathcal{U}_{\mathrm{ad}}=\mathcal{U}_{\mathrm{DN}} \text {, and } \Theta_{\mathrm{ad}}=\Theta_{\mathrm{DN}} .
$$

The main difficulty of the present situation lies in the weakly singular behavior of the solution $u_{\Omega}$ to (2.2) near $\Sigma_{D}$. In particular, the use of the formal Céa's method (see [15] and [6] for a comprehensive presentation), which implicitly relies on the smoothness of $u_{\Omega}$, gives rise to an erroneous shape derivative in the present context.

The conclusion of Proposition 3.2 was already observed in [8, 28], but our proof is slightly different: we rely on a direct calculation based on integration by parts.

Proposition 3.2. The functional $J(\Omega)$ is shape differentiable at any admissible shape $\Omega \in \mathcal{U}_{\mathrm{DN}}$, and its shape derivative reads (volumetric form):

$$
\begin{aligned}
\forall \theta \in \Theta_{\mathrm{DN}}, \quad J^{\prime}(\Omega)(\theta)=\int_{\partial \Omega}\left(j\left(u_{\Omega}\right)-\right. & \left.f p_{\Omega}\right) \theta \cdot n d s-\int_{\Omega} j^{\prime}\left(u_{\Omega}\right) \nabla u_{\Omega} \cdot \theta d x \\
& +\int_{\Omega}\left((\operatorname{div} \theta) \mathrm{I}-\nabla \theta-\nabla \theta^{T}\right) \nabla u_{\Omega} \cdot \nabla p_{\Omega} d x+\int_{\Omega} f \nabla p_{\Omega} \cdot \theta d x,
\end{aligned}
$$

where the adjoint state $p_{\Omega}$ is the unique solution in $H_{\Gamma_{D}}^{1}(\Omega)$ to the system:

$$
\left\{\begin{array}{cl}
-\Delta p_{\Omega}=-j^{\prime}\left(u_{\Omega}\right) & \text { in } \Omega, \\
p_{\Omega}=0 & \text { on } \Gamma_{D}, \\
\frac{\partial p_{\Omega}}{\partial n}=0 & \text { on } \Gamma_{N} \cup \Gamma .
\end{array}\right.
$$

Moreover, under the assumption (2.3), let us write the local structure of $u_{\Omega}$ and $p_{\Omega}$ in an open neighborhood $V^{i}$ of $s_{i}, i=0,1$ as follows:

$$
u_{\Omega}=u_{s}^{i}+u_{r}^{i} \text { and } p_{\Omega}=p_{s}^{i}+p_{r}^{i}
$$

in the above formula, $u_{r}^{i}, p_{r}^{i} \in H^{2}\left(V^{i}\right)$ and the weakly singular functions $u_{s}^{i}$ and $p_{s}^{i} \in H^{1}\left(V^{i}\right)$ have the following expressions in local polar coordinates centered at $s_{i}$ :

$$
u_{s}^{i}(r, \nu)=c_{u}^{i} r^{\frac{1}{2}} \cos \left(\frac{\nu}{2}\right), \text { and } p_{s}^{i}(r, \nu)=c_{p}^{i} r^{\frac{1}{2}} \cos \left(\frac{\nu}{2}\right), \text { if } n_{\Sigma_{D}}\left(s_{i}\right)=e_{1},
$$

where $\left(e_{1}, e_{2}\right)$ is the canonical basis of the plane. Then (3.13) rewrites (surface integral form):

$J^{\prime}(\Omega)(\theta)=\int_{\Gamma_{D} \cup \Gamma}\left(j\left(u_{\Omega}\right)-f p_{\Omega}\right) \theta \cdot n d s-\int_{\Gamma_{D}} \frac{\partial p_{\Omega}}{\partial n} \frac{\partial u_{\Omega}}{\partial n} \theta \cdot n d s+\int_{\Gamma} \frac{\partial u_{\Omega}}{\partial \tau} \frac{\partial p_{\Omega}}{\partial \tau} \theta \cdot n d s+\frac{\pi}{4} \sum_{i=0,1} c_{u}^{i} c_{p}^{i}\left(\theta \cdot n_{\Sigma_{D}}\right)\left(s_{i}\right)$. 
Remark 3.3. In the surface formula (3.18), the integrals

$$
-\int_{\Gamma_{D}} \frac{\partial p_{\Omega}}{\partial n} \frac{\partial u_{\Omega}}{\partial n} \theta \cdot n d s+\int_{\Gamma} \frac{\partial u_{\Omega}}{\partial \tau} \frac{\partial p_{\Omega}}{\partial \tau} \theta \cdot n d s
$$

are not well-defined individually, since they may blow up around the points $s_{i}$, as is quite clear from the look of the structure (3.16) and (3.17) of the singular parts of $u_{\Omega}$ and $p_{\Omega}$. However, these integrals turn out to have compensating singularities at $s_{i}$, so that their sum is well-defined as a Cauchy principal value; see the proof below.

Proof. The calculation of the volumetric formula (3.13) unfolds almost as in the case of Proposition 3.1, and we focus on the derivation of the surface formula (3.18), assuming that (2.3) holds. Again, the main idea of the calculation is to perform integration by parts from (3.13), taking advantage of the smoothness of $u_{\Omega}$ and $p_{\Omega}$ far from the singularities at $s_{i}, i=0,1$, and of the local structure (3.15) of these functions in the vicinity of $s_{i}$.

Let $\theta \in \Theta_{\mathrm{DN}}$ be fixed; for small $\delta>0$, let $B^{i}(\delta):=B\left(s_{i}, \delta\right)$ be the ball centered at $s_{i}$ with radius $\delta$, and let $\Omega_{\delta}:=\Omega \backslash\left(\overline{B^{0}(\delta)} \cup\left(\overline{B^{1}(\delta)}\right)\right.$. Since $u_{\Omega}$ and $p_{\Omega}$ belong to $H^{1}(\Omega)$, it holds from (3.13):

where:

$$
J^{\prime}(\Omega)(\theta)=\int_{\partial \Omega}\left(j\left(u_{\Omega}\right)-f p_{\Omega}\right) \theta \cdot n d s+\lim _{\delta \rightarrow 0} I_{\delta},
$$

$$
I_{\delta}:=-\int_{\Omega_{\delta}} j^{\prime}\left(u_{\Omega}\right) \nabla u_{\Omega} \cdot \theta d x+\int_{\Omega_{\delta}}\left(\operatorname{div} \theta \mathrm{I}-\nabla \theta-\nabla \theta^{T}\right) \nabla u_{\Omega} \cdot \nabla p_{\Omega} d x+\int_{\Omega_{\delta}} f \nabla p_{\Omega} \cdot \theta d x
$$

Using the smoothness of $u_{\Omega}$ and $p_{\Omega}$ on $\Omega_{\delta}$, the fact that $\theta$ vanishes on $\Gamma_{N}$, the definitions of $u_{\Omega}, p_{\Omega}$ and an integration by parts on the second term in the above right-hand side based on the identity (3.10), we obtain:

$$
I_{\delta}=\int_{\partial \Omega_{\delta}}\left(\nabla u_{\Omega} \cdot \nabla p_{\Omega} \theta \cdot n-\frac{\partial u_{\Omega}}{\partial n} \nabla p_{\Omega} \cdot \theta-\frac{\partial p_{\Omega}}{\partial n} \nabla u_{\Omega} \cdot \theta\right) d s
$$

To proceed further, we decompose the boundary $\partial \Omega_{\delta}$ as the disjoint reunion:

$$
\partial \Omega_{\delta}=\Gamma_{D, \delta} \cup \Gamma_{\delta} \cup \Gamma_{N} \cup \gamma_{0, \delta} \cup \gamma_{1, \delta},
$$

where $\Gamma_{D, \delta}=\Gamma_{D} \cap \Omega_{\delta}, \Gamma_{\delta}=\Gamma \cap \Omega_{\delta}$, and $\gamma_{i, \delta}=\partial B^{i}(\delta) \cap \Omega$ is the half-circle with center $s_{i}$ and radius $\delta$. We also denote by $s_{i, \delta}$ the intersection point between $\partial B^{i}(\delta)$ and $\Gamma$; see Fig. 2 about these notations.

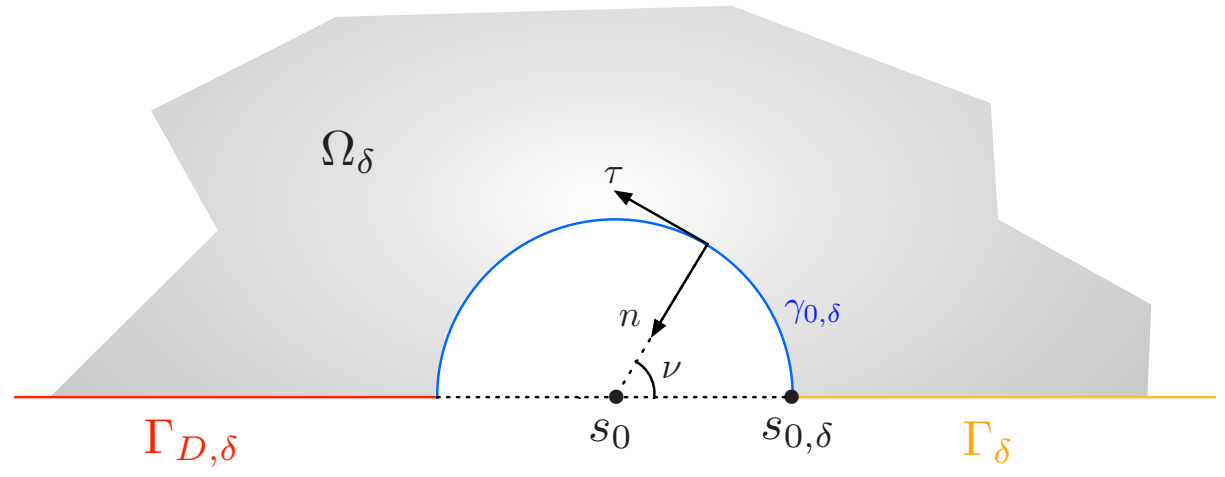

FIGURE 2. The local situation around the point $s_{0}$ in the proof of Proposition 3.2.

Since $\theta=0$ on $\Gamma_{N}$, it follows that:

$$
I_{\delta}=\int_{\Gamma_{\delta}} \frac{\partial u_{\Omega}}{\partial \tau} \frac{\partial p_{\Omega}}{\partial \tau} \theta \cdot n d s-\int_{\Gamma_{D, \delta}} \frac{\partial u_{\Omega}}{\partial n} \frac{\partial p_{\Omega}}{\partial n} \theta \cdot n d s+\sum_{i=0,1} \int_{\gamma_{i, \delta}} K\left(u_{\Omega}, p_{\Omega}\right) d s
$$

where we have introduced the shorthand:

$$
K(v, w)=\nabla v \cdot \nabla w \theta \cdot n-\frac{\partial v}{\partial n} \nabla w \cdot \theta-\frac{\partial w}{\partial n} \nabla v \cdot \theta .
$$


Let us now evaluate the contributions of $I_{\delta}$ on $\gamma_{i, \delta}$ for $i=0,1$ in the expression (3.20). Without loss of generality, we only deal with $i=0$, and we assume that $s_{0}=0$; according to $(2.3)$, $\partial \Omega$ is horizontal in the neighborhood of $s_{0}$ and we also assume that $\Gamma_{D}$ (resp. $\Gamma$ ) lies on the left-hand side (resp. the right-hand side) of $s_{0}$; see again Fig. 2. Introducing the polar coordinates $(r, \nu)$ with origin at $s_{0}=0$, taking into account our conventions for $\tau$ and $n$, we have:

$$
n=-\cos \nu e_{1}-\sin \nu e_{2}, \tau=-\sin \nu e_{1}+\cos \nu e_{2},
$$

and as far as derivatives are concerned:

$$
\frac{\partial}{\partial n}=-\frac{\partial}{\partial r}, \text { and } \frac{\partial}{\partial \tau}=\frac{1}{r} \frac{\partial}{\partial \nu}
$$

Recalling the local expressions (3.15) of $u_{\Omega}$ and $p_{\Omega}$ around $s_{0}$, it is easily seen that the only possibly non vanishing contribution of $\int_{\gamma_{0}, \delta} K\left(u_{\Omega}, p_{\Omega}\right) d s$ in the limit $\delta \rightarrow 0$ is given by the most singular part of its integrand:

$$
\lim _{\delta \rightarrow 0} \int_{\gamma_{0, \delta}} K\left(u_{\Omega}, p_{\Omega}\right) d s=\lim _{\delta \rightarrow 0} \widetilde{I_{\delta}^{0}}, \text { where } \widetilde{I_{\delta}^{0}}:=\int_{\gamma_{0, \delta}} K\left(u_{s}^{0}, p_{s}^{0}\right) d s .
$$

Let us then calculate the last integral. We have, on $\gamma_{0, \delta}$ :

$$
\begin{aligned}
\left(\nabla u_{s}^{0} \cdot \nabla p_{s}^{0}\right)(\theta \cdot n) & =\left(\frac{\partial u_{s}^{0}}{\partial \tau} \frac{\partial p_{s}^{0}}{\partial \tau}+\frac{\partial u_{s}^{0}}{\partial n} \frac{\partial p_{s}^{0}}{\partial n}\right)(\theta \cdot n) \\
& =\left(\frac{1}{r^{2}} \frac{\partial u_{s}^{0}}{\partial \nu} \frac{\partial p_{s}^{0}}{\partial \nu}+\frac{\partial u_{s}^{0}}{\partial r} \frac{\partial p_{s}^{0}}{\partial r}\right)(\theta \cdot n) \\
& =\left(\frac{c_{u}^{0} c_{p}^{0}}{4 r}\right)(\theta \cdot n)
\end{aligned}
$$

Likewise,

$$
\begin{aligned}
-\frac{\partial u_{s}^{0}}{\partial n} \nabla p_{s}^{0} \cdot \theta & =-\frac{\partial u_{s}^{0}}{\partial n} \frac{\partial p_{s}^{0}}{\partial \tau} \theta \cdot \tau-\frac{\partial u_{s}^{0}}{\partial n} \frac{\partial p_{s}^{0}}{\partial n} \theta \cdot n \\
& =\frac{1}{r} \frac{\partial u_{s}^{0}}{\partial r} \frac{\partial p_{s}^{0}}{\partial \nu} \theta \cdot \tau-\frac{\partial u_{s}^{0}}{\partial r} \frac{\partial p_{s}^{0}}{\partial r} \theta \cdot n \\
& =-\frac{c_{u}^{0} c_{p}^{0}}{4 r} \cos \left(\frac{\nu}{2}\right) \sin \left(\frac{\nu}{2}\right) \theta \cdot \tau-\frac{c_{u}^{0} c_{p}^{0}}{4 r} \cos ^{2}\left(\frac{\nu}{2}\right) \theta \cdot n
\end{aligned}
$$

and

$$
-\frac{\partial p_{s}^{0}}{\partial n} \nabla u_{s}^{0} \cdot \theta=-\frac{\partial u_{s}^{0}}{\partial n} \nabla p_{s}^{0} \cdot \theta=-\frac{c_{u}^{0} c_{p}^{0}}{4 r} \cos \left(\frac{\nu}{2}\right) \sin \left(\frac{\nu}{2}\right) \theta \cdot \tau-\frac{c_{u}^{0} c_{p}^{0}}{4 r} \cos ^{2}\left(\frac{\nu}{2}\right) \theta \cdot n .
$$

Gathering (3.21) to (3.23), we now obtain:

$$
\begin{aligned}
K\left(u_{s}^{0}, p_{s}^{0}\right) & =\frac{c_{u}^{0} c_{p}^{0}}{4 r_{0}}\left(\left(1-2 \cos ^{2}\left(\frac{\nu}{2}\right)\right) \theta \cdot n-2 \cos \left(\frac{\nu}{2}\right) \sin \left(\frac{\nu}{2}\right) \theta \cdot \tau\right) \\
& =\frac{c_{u}^{0} c_{p}^{0}}{4 r_{0}}(-(\sin \nu) \theta \cdot \tau-(\cos \nu) \theta \cdot n) \\
& =\frac{c_{u}^{0} c_{p}^{0}}{4 r} \theta_{1}
\end{aligned}
$$

where $\theta_{1}$ is the horizontal component of $\theta=\theta_{1} e_{1}+\theta_{2} e_{2}$. Therefore:

$$
\widetilde{I_{\delta}^{0}}=\left(\int_{0}^{\pi} \frac{c_{u}^{0} c_{p}^{0}}{4} \theta_{1}(\delta \cos \nu, \delta \sin \nu) d \nu\right) \stackrel{\delta \rightarrow 0}{\longrightarrow} \frac{\pi c_{u}^{0} c_{p}^{0}}{4} \theta_{1}(0)
$$

Combining all these results, we obtain the surface form $(3.18)$ of the shape derivative $J^{\prime}(\Omega)(\theta)$.

Remark 3.4. The result extends to the case where the boundary $\partial \Omega$ is not flat (but is still smooth) in the neighborhood of $\Sigma_{D}=\left\{s_{0}, s_{1}\right\}$. More precisely, let $V$ be a small enough neighborhood of either of the $s_{i}$, and let us introduce a local description of $\Omega$ as a graph, assuming for simplicity that $s_{i}=0$ and $n\left(s_{i}\right)=-e_{2}$ : $U$ is a neighborhood of 0 in $\mathbb{R}^{2}$ and $\psi\left(x_{1}, x_{2}\right)=\left(x_{1}, \varphi\left({ }_{2}\right)\right)$ is a smooth diffeomorphism from $U$ onto $V$ such that:

$$
\Omega \cap V=\left\{x=\left(x_{1}, x_{2}\right) \in \mathbb{R}^{2}, x_{2}>\varphi\left(x_{1}\right)\right\} \cap U .
$$

Then, it follows from [30], §5.2 that $u_{\Omega}$ reads in this case:

$$
u_{\Omega}=c^{i} S^{i} \circ \psi^{-1}+u_{r}^{i} \text { on } B_{\delta}\left(s_{i}\right),
$$

where $u_{r}^{i} \in H^{2}(V)$. The proof extends to this latter context then.

\section{Remark 3.5.}


- Interestingly, if $u_{\Omega}$ and $p_{\Omega}$ are assumed to be actually smooth (say $H^{2}$ ) in the neighborhood of the transition points $s_{0}$ and $s_{1}$, the shape derivative (3.18) no longer involves any term related to the geometry of the repartition of $\Gamma_{D}$ and $\Gamma$. In other terms, all the information about the sensitivity of $J(\Omega)$ with respect to the repartition of $\Gamma_{D}$ and $\Gamma$ is encoded in the (weak) singularities of $u_{\Omega}$ and $p_{\Omega}$.

- The fact that the sensitivity of $J(\Omega)$ with respect to variations of $\Sigma_{D}$ in (3.18) involves the singular behavior of $u_{\Omega}$ and $p_{\Omega}$ near $\Sigma_{D}$ is reminiscent of the field of fracture mechanics. There, the so-called Irwin formula relates the singularity of the elastic displacement of a material at the tip of a crack to the energy release rate guiding the growth of the crack; see [43].

\section{An approximate shape optimization problem to Deal with the Dirichlet-Neumann TRANSITION}

We have calculated in Section 3 the shape derivative of the functional $J(\Omega)$ given by (2.5), in the situation where either the transition $\Sigma_{N}$ or $\Sigma_{D}$ is subject to optimization. The resulting expression in the former case (see Proposition 3.1) may be readily used in a typical gradient-based shape optimization algorithm; see Section 5 .

On the other hand, in the case where $\Sigma_{D}$ is optimized, the expression supplied by Proposition 3.2 is unfortunately awkward from both the theoretical and practical perspectives. Indeed,

- The calculation of the surface form (3.18) of $J^{\prime}(\Omega)(\theta)$ was enabled by the precise knowledge of the local behavior (3.15) of $u_{\Omega}$ and $p_{\Omega}$ near the singularities $s_{0}$ and $s_{1}$. In more involved situations, for instance in three space dimensions, or in more challenging physical contexts (such as those of the linearized elasticity system, or the Stokes equations), such precise information may not be available, or may be difficult to handle.

- The numerical evaluation of the shape derivative $J^{\prime}(\Omega)(\theta)$ requires the calculation of the coefficients $c_{u}$ and $c_{p}$ featured in (3.15); this is doable, but it demands adapted numerical techniques, for instance an enrichment of the finite element basis with the singular functions, or adapted $\mathrm{p} / \mathrm{hp}$ finite mesh refinement methods; see [9, 27, 39] and the references therein. In our numerical setting, presented in Section 5, such techniques are bound to be all the more difficult to carry out that the boundary $\Sigma_{D}$ is not explicitly discretized in the computational mesh.

- Eventually, it is possible in principle to rely only on the volumetric form (3.13) of the shape derivative for algorithmic purposes, as is suggested for instance in [35, 29] and the references therein; nevertheless, for many practical purposes, it is interesting to have a surface expression for this shape derivative - for instance when it comes to using advanced optimization algorithms such as that introduced in [26].

We thenceforth focus our efforts on the instance of the problem (2.4) where this transition zone $\Sigma_{D}$ is also subject to optimization (while $\Sigma_{N}$ is not). To overcome the aforementioned difficulties, we introduce an approximation method which allows for the optimization of the boundary $\Sigma_{D}$ between regions bearing homogeneous Dirichlet and Neumann boundary conditions, without requiring the knowledge of the weakly singular behavior of $u_{\Omega}$ (and that of the adjoint state $p_{\Omega}$ ) in the neighborhood of $\Sigma_{D}$. As we shall see in Section 5, this method lends itself to an easy generalization to more difficult situations: transitions between other types of boundary conditions involving a singular state, other physical contexts than that of the Laplace equation, etc.

Throughout this section, unless stated otherwise, we consider the shape optimization problem (2.4) in the physical setting of Section 2, in the particular case where the transition $\Sigma_{D}$ between the regions $\Gamma_{D}$ and $\Gamma$ of $\partial \Omega$ is subject to optimization: $\mathcal{U}_{\mathrm{ad}}=\mathcal{U}_{\mathrm{DN}}$ and $\Theta_{\mathrm{ad}}=\Theta_{\mathrm{DN}}$. After introducing a few notations and background material regarding the notion of geodesic distance function in Section 4.1, in Section 4.2, we present an approximate version of the physical problem (2.2), relying on a 'small' parameter $\varepsilon>0$, with the noticeable feature that its unique solution $u_{\Omega, \varepsilon}$ is smooth. This leads to the introduction of an approximate shape optimization problem of a smoothed functional $J_{\varepsilon}(\Omega)$ in Section 4.3; we calculate the shape derivative $J_{\varepsilon}^{\prime}(\Omega)(\theta)$ by classical means, and the numerical evaluation of the resulting expression poses no particular difficulty. Finally, in Section 4.4, we prove in the model context where (2.3) holds that the approximate shape optimization problem converges to its exact counterpart, in the sense that $u_{\Omega, \varepsilon} \rightarrow u_{\Omega}$ as $\varepsilon \rightarrow 0$, and the values of $J_{\varepsilon}(\Omega)$ and $J_{\varepsilon}^{\prime}(\Omega)(\theta)$ converge to their exact counterparts $J(\Omega)$ and $J^{\prime}(\Omega)(\theta)$. 


\subsection{About the signed distance function to a subset on a manifold}

In this section, we collect some material about the signed distance function to a subregion of an oriented, closed and smooth submanifold $\mathcal{M}$ of $\mathbb{R}^{d}$ with codimension $1 ; \mathcal{M}$ is equipped with a Riemannian structure by endowing its tangent bundle with the inner product induced by that of $\mathbb{R}^{d}$ and we denote by $n$ its unit normal vector. In the context of Section $2, \mathcal{M}$ stands for the boundary $\partial \Omega$ of the considered shape $\Omega$.

Let us first set some notations and recall some necessary background material from Riemannian geometry; for these matters, we refer to [25].

The length $\ell(\gamma)$ of a piecewise differentiable curve $\gamma: I \rightarrow \mathcal{M}$ defined on an interval $I \subset \mathbb{R}$ is

$$
\ell(\gamma)=\int_{I}\left|\gamma^{\prime}(t)\right| d t
$$

The geodesic distance $d^{\mathcal{M}}(x, y)$ between two points $x, y \in \mathcal{M}$ is then:

$$
d^{\mathcal{M}}(x, y)=\inf \ell(\gamma)
$$

where the infimum is taken over all piecewise differentiable curves $\gamma:(a, b) \rightarrow \mathcal{M}$ such that $\gamma(a)=x$ and $\gamma(b)=y$. Likewise, we denote by

$$
d^{\mathcal{M}}(x, K)=\inf _{y \in K} d^{\mathcal{M}}(x, y)
$$

the distance between $x \in \mathcal{M}$ and a subset $K \subset \mathcal{M}$.

The notion of distance on $\mathcal{M}$ is conveniently described in terms of the exponential mapping: for any point $p \in \mathcal{M}$ and vector $v$ in the tangent plane $T_{p} \mathcal{M}$ to $\mathcal{M}$ at $p$, $\exp _{p}(v)$ stands for the point $\gamma(1, p, v)$, where $t \mapsto \gamma(t, p, v)$ is the unique geodesic curve on $\mathcal{M}$ such that

$$
\gamma(0)=p, \text { and } \gamma^{\prime}(0)=v .
$$

As a well-known fact in Riemannian geometry, the mapping $\exp _{p}$ is well-defined on an open neighborhood $U$ of 0 in $T_{p} \Gamma$ and it is a diffeomorphism from $U$ onto an open neighborhood $V$ of $p$ in $\mathcal{M}$. The reciprocal mapping is the logarithm $\log _{p}: V \rightarrow U$ :

$$
\forall y \in V, \log _{p}(y)=\text { the unique } v \in U \text { s.t. } \gamma(1, p, v)=y .
$$

We now turn to the notion of signed distance function on the submanifold $\mathcal{M}$ : let $G \subset \mathcal{M}$ be an open subset which we assume to be smooth for simplicity; its boundary $\Sigma:=\partial G$ is a closed, smooth submanifold of $\mathbb{R}^{d}$ with codimension 2 , and we denote by $n_{\Sigma}: \Sigma \rightarrow \mathbb{S}^{1}$ the unit normal vector to $\Sigma$ pointing outward $G$. In particular, $n_{\Sigma}$ is a vector field along $\Sigma$ which is tangential to $\mathcal{M}$.

Definition 4.1. The signed distance function $d_{G}$ to $G$ is defined by:

$$
\forall x \in \mathcal{M}, d_{G}(x)=\left\{\begin{array}{cl}
-d^{\mathcal{M}}(x, \Sigma) & \text { if } x \in G, \\
0 & \text { if } x \in \Sigma, \\
d^{\mathcal{M}}(x, \Sigma) & \text { if } x \in \mathcal{M} \backslash \bar{G} .
\end{array}\right.
$$

For $y \in \mathcal{M}$, we denote by $p_{\Sigma}(y)$ the projection of $y$ onto $\Sigma$, that is, the unique point $x \in \Sigma$ such that $d^{\mathcal{M}}(x, y)=d^{\mathcal{M}}(x, \Sigma)$, when this makes sense (i.e. when there is indeed such a unique point).

The following theorem states in essence that, when $G$ is a regular subset of $\mathcal{M}$, the projection mapping $p_{\Sigma}$ is well-defined and smooth on a neighborhood of $\Sigma$. The proof is essentially the same as that of Theorem 3.1 in [7]: it is a local argument relying on the implicit function theorem, and we omit the details for brevity.

Theorem 4.1. Let $G$ be a regular open subset of $\mathcal{M}$; then there exists an open neighborhood $U \subset \mathcal{M}$ of $\Sigma$ such that the mapping

$$
\Sigma \times\left(-t_{0}, t_{0}\right) \rightarrow U, \mathcal{F}(x, t)=\exp _{x}\left(\operatorname{tn}_{\Sigma}(x)\right)
$$

is a diffeomorphism of class $\mathcal{C}^{\infty}$. For $y=\mathcal{F}(x, t) \in U$, one has:

$$
t=d_{G}(y), \text { and } x=p_{\Sigma}(y) .
$$

Let us now state a result about the (tangential) gradient of the distance function; the proof of the first point lies e.g. in [54], while the second one follows from a direct application of the theorem of differentiation of a minimum value (see [23], Chap. 10, Th. 2.1):

\section{Proposition 4.1.}


- Let $p \in \mathcal{M}$; consider the function $\rho(x):=d^{\mathcal{M}}(x, p)$ on a neighborhood $U$ of $p$. Then $\rho$ is differentiable on $U \backslash\{p\}$ and its (tangential) gradient reads:

$$
\nabla \rho(x)=-\frac{1}{d^{\mathcal{M}}(x, p)} \log _{x}(p) .
$$

- Let $G$ be a regular open subset of $\mathcal{M}$ with boundary $\Sigma$, and let $U$ be the neighborhood supplied by Theorem 4.1. Then the signed distance function $d_{G}$ is differentiable at any point $x \in U$, and its gradient reads:

$$
\nabla d_{G}(x)=-\frac{1}{d_{G}(x)} \log _{x}\left(p_{\Sigma}(x)\right) .
$$

We eventually consider the differentiation of the signed distance function $d_{G}$ with respect to variations of the manifold $\mathcal{M}$ (and thus of $\Sigma$ ). The following result is new to the best of our knowledge:

Proposition 4.2. Let $G$ be a regular open subset of $\mathcal{M}$, and let $U$ be the neighborhood of $\Sigma=\partial G$ supplied by Theorem 4.1. consider a fixed point $y \in U \backslash \Sigma$, and let $p=p_{\Sigma}(y)$, so that $\sigma(t)=\exp _{p}\left(\operatorname{tn}_{\Sigma}(p)\right)$ is the unique geodesic joining $p$ to $y$, parametrized by arc length. For any vector field $\theta \in \mathcal{C}^{1, \infty}\left(\mathbb{R}^{d}, \mathbb{R}^{d}\right)$, we define the variations of $\mathcal{M}$ and $G$ according to Hadamard's method (see Section 2.2):

$$
\mathcal{M}_{\theta}=(\operatorname{Id}+\theta)(\mathcal{M}) \text {, and } G_{\theta}=(\operatorname{Id}+\theta)(G) .
$$

Define:

$$
D(\theta)=d_{G_{\theta}}(y+\theta(y))
$$

Then $D$ is Fréchet differentiable at $\theta=0$ and its derivative reads:

$$
D^{\prime}(0)(\theta)=-\theta(y) \cdot \frac{\log _{y}(p)}{d_{G}(y)}-\theta(p) \cdot n_{\Sigma}(p)+\int_{0}^{d_{G}(y)} \Pi_{\sigma(t)}^{\mathcal{M}}\left(\sigma^{\prime}(t), \sigma^{\prime}(t)\right)(\theta \cdot n)(\sigma(t)) d t .
$$

In the above formula, $\Pi_{p}^{\mathcal{M}}$ is the second fundamental form of $\mathcal{M}$ at $p$, that is:

$$
\forall v \in T_{p} \mathcal{M}, \Pi_{p}^{\mathcal{M}} v \cdot v=-\nabla n(p) v \cdot v
$$

where $n$ is any extension of the normal vector $n: \mathcal{M} \rightarrow \mathbb{R}^{d}$ to an open neighborhood of $\mathcal{M}$ in $\mathbb{R}^{d}$.

Proof. Assume for simplicity that $y \in \mathcal{M} \backslash \bar{G}$, the result being proved in a similar same way if $y \in G$. From the definition of $D(\theta)$, one has:

$$
\begin{aligned}
D(\theta) & =\inf _{x \in \Sigma} \inf _{\substack{\gamma:(a, b) \rightarrow \mathcal{M}_{\theta} \\
\gamma(a)=x+\theta(x), \gamma(b)=y+\theta(y)}} \int_{a}^{b}\left|\gamma^{\prime}(t)\right| d t, \\
& =\inf _{x \in \Sigma} \inf _{\substack{\gamma:(a, b) \rightarrow \mathcal{M} \\
\gamma(a)=x, \gamma(b)=y}} \int_{a}^{b} \sqrt{\left(\mathrm{I}+\nabla \theta^{T}(\gamma(t))\right)(I+\nabla \theta(\gamma(t))) \gamma^{\prime}(t) \cdot \gamma^{\prime}(t)} d t,
\end{aligned}
$$

and we know that, for $\theta=0$, this infimum is attained uniquely for $x=p$ and $\gamma=\sigma$, the geodesic curve joining $p$ to $y$. Therefore, the theorem of differentiation through a minimum (see again [23], Chap. 10, Th. 2.1) entails that $D$ is differentiable at $\theta=0$ and:

$$
D^{\prime}(0)(\theta)=\int_{a}^{b} \frac{1}{\left|\sigma^{\prime}(t)\right|} \nabla \theta(\sigma(t)) \sigma^{\prime}(t) \cdot \sigma^{\prime}(t) d t
$$

Since $\sigma(t)=\exp _{p}\left(\operatorname{tn}_{\Sigma}(p)\right)$ is parametrized by arc length, we have in particular $\left|\sigma^{\prime}(t)\right|=1, \sigma(0)=p$ and $\sigma\left(d_{G}(y)\right)=y$ (that is, $a=0$ and $b=d_{G}(y)$ in the above formula); therefore,

$$
D^{\prime}(0)(\theta)=\int_{0}^{d_{G}(y)} \nabla \theta(\sigma(t)) \sigma^{\prime}(t) \cdot \sigma^{\prime}(t) d t=\int_{0}^{d_{G}(y)}(\theta(\sigma(t)))^{\prime} \cdot \sigma^{\prime}(t) d t
$$

whence, using integration by parts:

$$
D^{\prime}(0)(\theta)=\theta(y) \cdot \sigma^{\prime}\left(d_{G}(y)\right)-\theta(p) \cdot \sigma^{\prime}(0)-\int_{0}^{d_{G}(y)} \theta(\sigma(t)) \cdot \sigma^{\prime \prime}(t) d t
$$


In the above formula, it follows from the definition of $\sigma$ and Proposition 4.1 that:

$$
\sigma^{\prime}(0)=n_{\Sigma}(p), \text { and } \sigma^{\prime}\left(d_{G}(y)\right)=-\frac{\log _{y}(p)}{d_{G}(y)} .
$$

On the other hand, it follows from the fact that $\sigma$ is a geodesic that:

$$
\sigma^{\prime \prime}(t) \cdot v=0 \text { for any tangent vector } v \in T_{\sigma(t)} \mathcal{M} \text {; }
$$

see [25], Chap. 2, Exercise 4. Since in addition $\sigma^{\prime}(t) \cdot n(\sigma(t))=0$, differentiation with respect to $t$ yields:

$$
\sigma^{\prime \prime}(t) \cdot n(\sigma(t))=-\nabla n(\sigma(t)) \sigma^{\prime}(t) \cdot \sigma^{\prime}(t)=\Pi_{\sigma(t)}^{\mathcal{M}}\left(\sigma^{\prime}(t), \sigma^{\prime}(t)\right),
$$

which terminates the proof.

\section{Remark 4.1.}

- The formula (4.1) is of 'Lagrangian' nature: for given $y \in \mathcal{M}$, the derivative of the distance function to the perturbed set $G_{\theta}$ at the perturbed point $y+\theta(y)$ is calculated. Using the more classical notation $\dot{d}_{G}(\theta)(y)=D^{\prime}(0)(\theta)$ for this derivative, the corresponding Eulerian derivative $d_{G}^{\prime}(\theta)(y)$ of $d_{G}$ at $y$ is then defined by the formula:

$$
\begin{aligned}
d_{G}^{\prime}(\theta)(y) & :=\stackrel{\circ}{d_{G}}(\theta)(y)-\nabla d_{G}(y) \cdot \theta \\
& =-\theta(p) \cdot n_{\Sigma}(p)+\int_{0}^{d_{G}(y)} \Pi_{\sigma(t)}^{\mathcal{M}}\left(\sigma^{\prime}(t), \sigma^{\prime}(t)\right)(\theta \cdot n)(\sigma(t)) d t,
\end{aligned}
$$

where we have used Proposition 4.1.

- The structure of (4.1) is quite intuitive: the first two terms are exactly those featured in the formula for the 'Lagrangian' derivative of the signed distance function in the Euclidean case, i.e. without taking into account the curvature of the ambient space (see e.g. [19]), while the last one expresses the deformation with respect to $\theta$ of the geodesic between $p$ and $y$ out of the (normal) variation of the manifold $\mathcal{M}$.

\subsection{The smoothed setting}

In the setting of Section 2 (see also Fig. 1), and following the works [4, 24], we trade the solution $u_{\Omega}$ to the 'exact' problem (2.2) for that $u_{\Omega, \varepsilon}$ to the following approximate version, where the homogeneous Dirichlet and Neumann boundary conditions on $\Gamma_{D}$ and $\Gamma$ respectively are replaced by a Robin boundary condition on $\Gamma_{D} \cup \Gamma$ :

$$
\left\{\begin{array}{cl}
-\Delta u_{\Omega, \varepsilon}=f & \text { in } \Omega \\
\frac{\partial u_{\Omega, \varepsilon}}{\partial n}+h_{\varepsilon} u_{\Omega, \varepsilon}=0 & \text { on } \Gamma \cup \Gamma_{D} \\
\frac{\partial u_{\Omega, \varepsilon}}{\partial n}=g & \text { on } \Gamma_{N} .
\end{array}\right.
$$

Here, the coefficient $h_{\varepsilon}$ is defined by:

$$
h_{\varepsilon}(x)=\frac{1}{\varepsilon} h\left(\frac{d_{\Gamma_{D}}(x)}{\varepsilon}\right),
$$

where $h: \mathbb{R} \rightarrow \mathbb{R}$ is a smooth function with the properties:

$$
0 \leq h \leq 1, h \equiv 1 \text { on }(-\infty,-1], h(0)>0, h \equiv 0 \text { on }[1, \infty),
$$

and $d_{\Gamma_{D}}(x)$ is the (geodesic) signed distance function to $\Gamma_{D}$ on the surface $\partial \Omega$; see Definition 4.1. In other words, $h_{\varepsilon}$ equals $\frac{1}{\varepsilon}$ inside $\Gamma_{D}$, 'far' from $\Sigma_{D}, 0$ on $\Gamma$ 'far' from $\Sigma_{D}$, and it presents a smooth transition between these two values in a tubular neighborhood of $\Sigma_{D}$ with (geodesic) width $\varepsilon$, so that the boundary conditions in (2.2) are approximately satisfied; see Fig. 3.

In particular, $h_{\varepsilon}$ vanishes on a neighborhood of $\Gamma_{N}$ in $\partial \Omega$; notice also that our assumptions on $h$ imply that there exists a real value $\alpha>0$ which is independent of $\varepsilon$ such that:

$$
\forall x \in \Gamma_{D}, \alpha \leq \varepsilon h_{\varepsilon}(x) .
$$

The variational formulation associated to (4.2) reads: $u_{\Omega, \varepsilon}$ is the unique function in $H^{1}(\Omega)$ such that

$$
\forall v \in H^{1}(\Omega), \int_{\Omega} \nabla u_{\Omega, \varepsilon} \cdot \nabla v d x+\int_{\Gamma_{D} \cup \Gamma} h_{\varepsilon} u_{\Omega, \varepsilon} v d s=\int_{\Omega} f v d x+\int_{\Gamma_{N}} g v d s .
$$




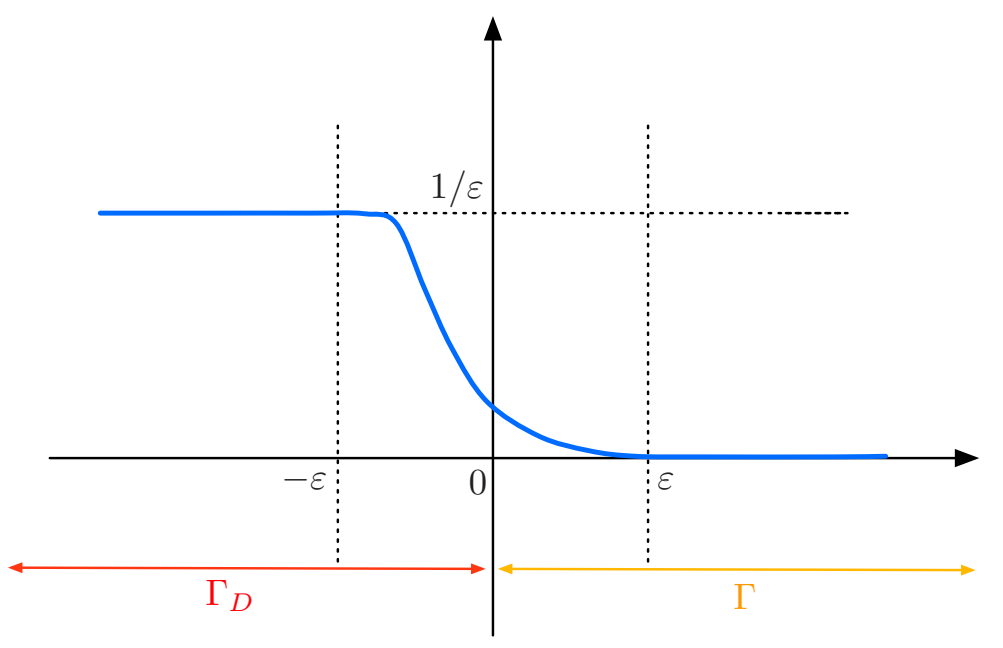

FiguRE 3. Graph of the function $h_{\varepsilon}$ defined by (4.3).

It follows from the standard Lax-Milgram theory that this problem is well-posed. In addition, for a fixed value of $\varepsilon>0$, due to the smoothness of $\Omega$ and $h_{\varepsilon}$ (see Section 4.1 about the smoothness of $d_{\Gamma_{D}}$ ), the solution $u_{\Omega, \varepsilon}$ to (4.2) actually enjoys $H^{2}$ regularity on a neighborhood of $\Sigma_{D}$, as a consequence of the standard regularity theory for elliptic equations; see e.g. [13], Chap. 9.

Remark 4.2. This approximation method can be straightforwardly adapted to different contexts, such as that of the linearized elasticity system; see Section 5 for illustrations, and [24] for an adaptation in the context of the acoustic Helmholtz equation.

\subsection{The approximate shape optimization problem}

We propose to replace the exact shape optimization problem (2.4) by its regularized counterpart:

$$
\inf _{\Omega \in \mathcal{U}_{\mathrm{DN}}} J_{\varepsilon}(\Omega), \text { where } J_{\varepsilon}(\Omega):=\int_{\Omega} j\left(u_{\Omega, \varepsilon}\right) d x,
$$

and $u_{\Omega, \varepsilon}$ is the solution to (4.2).

When it comes to the shape derivative of $J_{\varepsilon}(\Omega)$, the result of interest is the following:

Proposition 4.3. The functional $J_{\varepsilon}(\Omega)$ is shape differentiable at any admissible shape $\Omega \in \mathcal{U}_{\mathrm{DN}}$, and its shape derivative reads, for arbitrary $\theta \in \Theta_{\mathrm{DN}}$ (volumetric form):

$$
\begin{aligned}
& J_{\varepsilon}^{\prime}(\Omega)(\theta)=\int_{\partial \Omega}\left(j\left(u_{\Omega, \varepsilon}\right)-\right.\left.f p_{\Omega, \varepsilon}\right) \theta \cdot n d s-\int_{\Omega} j^{\prime}\left(u_{\Omega, \varepsilon}\right) \nabla u_{\Omega, \varepsilon} \cdot \theta d x+\int_{\Omega}\left(\operatorname{div} \theta \mathrm{I}-\nabla \theta-\nabla \theta^{T}\right) \nabla u_{\Omega, \varepsilon} \cdot \nabla p_{\Omega, \varepsilon} d x \\
&+\int_{\Gamma \cup \Gamma_{D}}\left(\operatorname{div}_{\partial \Omega}\right) \theta h_{\varepsilon} u_{\Omega, \varepsilon} p_{\Omega, \varepsilon} d s+\int_{\Omega} f \nabla p_{\Omega, \varepsilon} \cdot \theta d x \\
&+ \frac{1}{\varepsilon^{2}} \int_{\Gamma \cup \Gamma_{D}} h^{\prime}\left(\frac{d_{\Gamma_{D}}}{\varepsilon}\right)\left(-\theta(x) \cdot \frac{\log _{x}\left(p_{\Sigma_{D}}(x)\right)}{d_{\Gamma_{D}}(x)}-\theta\left(p_{\Sigma_{D}}(x)\right) \cdot n_{\Sigma_{D}}\left(p_{\Sigma_{D}}(x)\right)\right) u_{\Omega, \varepsilon} p_{\Omega, \varepsilon} d s(x) \\
& \frac{1}{\varepsilon^{2}} \int_{\Gamma \cup \Gamma_{D}} h^{\prime}\left(\frac{d_{\Gamma_{D}}}{\varepsilon}\right)\left(\int_{0}^{d_{\Gamma_{D}}(x)} \Pi_{\sigma_{x}(t)}^{\partial \Omega}\left(\sigma_{x}^{\prime}(t), \sigma_{x}^{\prime}(t)\right)(\theta \cdot n)\left(\sigma_{x}(t)\right) d t\right) u_{\Omega, \varepsilon} p_{\Omega, \varepsilon} d s(x),
\end{aligned}
$$


where $\sigma_{x}(t)=\exp _{{\sum_{\Sigma_{D}(x)}}}\left(\operatorname{tn}_{\Sigma_{D}}\left(p_{\Sigma_{D}}(x)\right)\right)$ is the unique geodesic passing through $x$ and $p_{\Sigma_{D}}(x)$, and the adjoint state $p_{\Omega, \varepsilon}$ is the unique solution in $H^{1}(\Omega)$ to the following system:

$$
\left\{\begin{array}{cl}
-\Delta p_{\Omega, \varepsilon}=-j\left(u_{\Omega, \varepsilon}\right) & \text { in } \Omega, \\
\frac{\partial p_{\Omega, \varepsilon}}{\partial n}+h_{\varepsilon} p_{\Omega, \varepsilon}=0 & \text { on } \Gamma_{D} \cup \Gamma, \\
\frac{\partial p_{\Omega, \varepsilon}}{\partial n}=0 & \text { on } \Gamma_{N} .
\end{array}\right.
$$

Equivalently, this rewrites (surface form):

$$
\begin{aligned}
& \text { (4.9) } J_{\varepsilon}^{\prime}(\Omega)(\theta)=\int_{\Gamma \cup \Gamma_{D}}\left(j\left(u_{\Omega, \varepsilon}\right)-f p_{\Omega, \varepsilon}+\nabla_{\partial \Omega} u_{\Omega, \varepsilon} \cdot \nabla_{\partial \Omega} p_{\Omega, \varepsilon}-\frac{\partial u_{\Omega, \varepsilon}}{\partial n} \frac{\partial p_{\Omega, \varepsilon}}{\partial n}-\kappa p_{\Omega, \varepsilon} \frac{\partial u_{\Omega, \varepsilon}}{\partial n}\right) \theta \cdot n d s \\
& +\frac{1}{\varepsilon^{2}} \int_{\Gamma \cup \Gamma_{D}} h^{\prime}\left(\frac{d_{\Gamma_{D}}}{\varepsilon}\right)\left(-\theta\left(p_{\Sigma_{D}}(x)\right) \cdot n_{\Sigma_{D}}\left(p_{\Sigma_{D}}(x)\right)+\int_{0}^{d_{\Gamma_{D}}(x)} \Pi_{\sigma_{x}(t)}^{\partial \Omega}\left(\sigma_{x}^{\prime}(t), \sigma_{x}^{\prime}(t)\right)(\theta \cdot n)\left(\sigma_{x}(t)\right) d t\right) u_{\Omega, \varepsilon} p_{\Omega, \varepsilon} d s(x) .
\end{aligned}
$$

Proof. Proof of (4.7): The proof is very similar to that of the volumetric formula (3.1) in Proposition 3.2, and we only sketch the main ingredients. At first, using the implicit function theorem, one sees that the solution $u_{\Omega, \varepsilon}$ to (4.2) has a 'Lagrangian derivative' $\stackrel{\circ}{\Omega}, \varepsilon_{\Omega}(\theta)$, which is the unique solution in $H^{1}(\Omega)$ to the following variational problem: for all $v \in H^{1}(\Omega)$,

$$
\begin{aligned}
& \int_{\Omega} \nabla \stackrel{\circ}{\Omega, \varepsilon}_{\Omega}(\theta) \cdot \nabla v d x+\int_{\Gamma \cup \Gamma_{D}} h_{\varepsilon}\left(d_{\Gamma_{D}}\right) \stackrel{\circ}{\Omega, \varepsilon}_{\Omega}(\theta) v d s= \\
& \int_{\Omega}\left(\operatorname{div}(f \theta) v+\left(\nabla \theta+\nabla \theta^{T}-\operatorname{div} \theta \mathrm{I}\right) \nabla u_{\Omega, \varepsilon} \cdot \nabla v\right) d x-\int_{\Gamma \cup \Gamma_{D}}\left(\operatorname{div}_{\partial \Omega} \theta\right) h_{\varepsilon}\left(d_{\Gamma_{D}}\right) u_{\Omega, \varepsilon} v d s \\
& -\frac{1}{\varepsilon^{2}} \int_{\Gamma \cup \Gamma_{D}} h^{\prime}\left(\frac{d_{\Gamma_{D}}}{\varepsilon}\right)\left(-\theta(x) \cdot \frac{\log _{x}\left(p_{\Sigma_{D}}(x)\right)}{d_{\Gamma_{D}}(x)}-\theta\left(p_{\Sigma_{D}}(x)\right) \cdot n_{\Sigma_{D}}\left(p_{\Sigma_{D}}(x)\right)\right) u_{\Omega, \varepsilon} v d s(x) \\
& \quad-\frac{1}{\varepsilon^{2}} \int_{\Gamma \cup \Gamma_{D}} h^{\prime}\left(\frac{d_{\Gamma_{D}}}{\varepsilon}\right)\left(\int_{0}^{d_{\Sigma_{D}}(x)} \Pi_{\gamma(t)}^{\partial \Omega}\left(\gamma^{\prime}(t), \gamma^{\prime}(t)\right)(\theta \cdot n)(\gamma(t)) d t\right) u_{\Omega, \varepsilon} v d s(x),
\end{aligned}
$$

where we have used Proposition 4.2 for the 'Lagrangian' derivative of the geodesic distance.

On the other hand, using a change of variables yields:

$$
J_{\varepsilon}\left(\Omega_{\theta}\right)=\int_{\Omega}|\operatorname{det}(\mathrm{I}+\nabla \theta)| j\left(u_{\Omega_{\theta}, \varepsilon} \circ(\mathrm{Id}+\theta)\right) d x,
$$

whence, differentiating with respect to $\theta$ and using the variational formulation for the adjoint system (4.8):

$$
\begin{aligned}
J_{\varepsilon}^{\prime}(\Omega)(\theta) & =\int_{\Omega} \operatorname{div} \theta j\left(u_{\Omega, \varepsilon}\right) d x+\int_{\Omega} j^{\prime}\left(u_{\Omega, \varepsilon}\right) \stackrel{\leftrightarrow}{u}_{\Omega, \varepsilon}(\theta) d x \\
& =\int_{\Omega} \operatorname{div} \theta j\left(u_{\Omega, \varepsilon}\right) d x-\int_{\Omega} \nabla \stackrel{\varkappa}{\Omega, \varepsilon}_{\Omega}(\theta) \cdot \nabla p_{\Omega, \varepsilon} d x \int_{\Gamma \cup \Gamma_{D}} h_{\varepsilon}\left(d_{\Gamma_{D}}\right) \stackrel{\circ}{\Omega, \varepsilon}_{\Omega, \varepsilon}(\theta) p_{\Omega, \varepsilon} d s
\end{aligned}
$$

Combining this with (4.10) eventually yields the desired result.

Proof of (4.9): To simplify notations, until the end of the proof, we take the shortcuts $u \equiv u_{\Omega, \varepsilon}$ and $p \equiv p_{\Omega, \varepsilon}$. We decompose the volumetric expression (4.7) as:

$$
J_{\varepsilon}^{\prime}(\Omega)(\theta)=I_{1}(\theta)+I_{2}(\theta),
$$

where

$$
\begin{array}{r}
I_{1}(\theta)=\int_{\partial \Omega}(j(u)-f p) \theta \cdot n d s-\int_{\Omega} j^{\prime}(u) \nabla u \cdot \theta d x+\int_{\Omega}\left(\operatorname{div} \theta \mathrm{I}-\nabla \theta-\nabla \theta^{T}\right) \nabla u \cdot \nabla p d x \\
+\int_{\Gamma \cup \Gamma_{D}} \operatorname{div}_{\partial \Omega} \theta h_{\Omega, \varepsilon} u p d s+\int_{\Omega} f \nabla p \cdot \theta d x,
\end{array}
$$

and

$$
I_{2}(\theta)=\frac{1}{\varepsilon^{2}} \int_{\Gamma \cup \Gamma_{D}} h^{\prime}\left(\frac{d_{\Gamma_{D}}}{\varepsilon}\right)\left(-\theta(x) \cdot \frac{\log _{x}\left(p_{\Sigma_{D}}(x)\right)}{d_{\Gamma_{D}}(x)}-\theta\left(p_{\Sigma_{D}}(x)\right) \cdot n_{\Sigma_{D}}\left(p_{\Sigma_{D}}(x)\right)\right) u p d s(x)
$$




$$
+\frac{1}{\varepsilon^{2}} \int_{\Gamma \cup \Gamma_{D}} h^{\prime}\left(\frac{d_{\Gamma_{D}}}{\varepsilon}\right)\left(\int_{0}^{d_{\Gamma_{D}}(x)} \Pi_{\sigma_{x}(t)}^{\partial \Omega}\left(\sigma_{x}^{\prime}(t), \sigma_{x}^{\prime}(t)\right)(\theta \cdot n)\left(\sigma_{x}(t)\right) d t\right) u p d s(x) .
$$

Let us first rearrange the expression of $I_{1}(\theta)$. To this end, using the same type of calculations as in the proofs of Propositions 3.1 and 3.2, integration by parts together with the fact that $u$ and $p$ have at least $H^{2}$ regularity near $\Gamma_{D} \cup \Gamma$ yield straightforwardly:

$$
\begin{aligned}
I_{1}(\theta)= & \int_{\partial \Omega}(j(u)-f p) \theta \cdot n d s-\int_{\Omega} j^{\prime}(u) \nabla u \cdot \theta d x+\int_{\Omega} f \nabla p \cdot \theta d x+\int_{\Gamma \cup \Gamma_{D}} \operatorname{div}_{\partial \Omega} \theta h_{\Omega, \varepsilon} u p d s \\
& +\int_{\Gamma \cup \Gamma_{D}}\left(\nabla u \cdot \nabla p \theta \cdot n-\frac{\partial u}{\partial n} \nabla p \cdot \theta-\frac{\partial p}{\partial n} \nabla u \cdot \theta\right) d s \\
& +\int_{\Omega}\left(-\nabla(\nabla u \cdot \nabla p)+\Delta u \nabla p+\Delta p \nabla u+\nabla^{2} p \nabla u+\nabla^{2} u \nabla p\right) \cdot \theta d x \\
= & \int_{\partial \Omega}(j(u)-f p) \theta \cdot n d s+\int_{\Gamma \cup \Gamma_{D}} \operatorname{div}_{\partial \Omega} \theta h_{\Omega, \varepsilon} u p d s+\int_{\Gamma \cup \Gamma_{D}}\left(\nabla u \cdot \nabla p \theta \cdot n-\frac{\partial u}{\partial n} \nabla p \cdot \theta-\frac{\partial p}{\partial n} \nabla u \cdot \theta\right) d s .
\end{aligned}
$$

Denoting by $\mathcal{D}$ the last integrand in the above right-hand side, we obtain:

$$
\begin{aligned}
\mathcal{D} & :=\nabla u \cdot \nabla p \theta \cdot n-\frac{\partial u}{\partial n} \nabla p \cdot \theta-\frac{\partial p}{\partial n} \nabla u \cdot \theta, \\
& =-\frac{\partial u}{\partial n} \frac{\partial p}{\partial n} \theta \cdot n-\left(\frac{\partial u}{\partial n} \nabla_{\partial \Omega} p \cdot \theta+\frac{\partial p}{\partial n} \nabla_{\partial \Omega} u \cdot \theta\right), \\
& =-\frac{\partial u}{\partial n} \frac{\partial p}{\partial n} \theta \cdot n+h_{\varepsilon}\left(u \nabla_{\partial \Omega} p \cdot \theta+p \nabla_{\partial \Omega} u \cdot \theta\right) .
\end{aligned}
$$

On the other hand, integrating by parts on the surface $\partial \Omega$ (see again Proposition A.1), we obtain:

$$
\begin{aligned}
\int_{\partial \Omega} \operatorname{div}_{\partial \Omega} \theta h_{\Omega, \varepsilon} u p d s=\int_{\Gamma_{D} \cup \Gamma} h_{\varepsilon} \kappa u p \theta \cdot n d s-\int_{\Gamma \cup \Gamma_{D}} h_{\varepsilon}\left(p \nabla_{\partial \Omega} u \cdot \theta+u \nabla_{\partial \Omega} p \cdot \theta\right) d s & -\frac{1}{\varepsilon^{2}} \int_{\Gamma \cup \Gamma_{D}} h^{\prime}\left(\frac{d_{\Gamma_{D}}}{\varepsilon}\right)\left(\nabla_{\partial \Omega} d_{\Gamma_{D}} \cdot \theta\right) u p d s .
\end{aligned}
$$

Finally, combining (4.11) to (4.13) with the definitions of $I_{1}(\theta)$ and $I_{2}(\theta)$, as well as Proposition 4.1 for the tangential gradient of the geodesic signed distance function, the desired result follows.

\subsection{Study of the convergence of the approximate model to the exact problem}

In this section, we are interested in evaluating in which capacity the exact shape optimization problem (2.4) is correctly approximated by its smoothed counterpart (4.6). More precisely, we investigate the convergence of the objective function $J_{\varepsilon}(\Omega)$ and that of its shape derivative $J_{\varepsilon}^{\prime}(\Omega)$ to the exact versions $J(\Omega)$ and $J^{\prime}(\Omega)$ respectively, for a fixed shape $\Omega \subset \mathbb{R}^{2}$. In order to keep the exposition as simple as possible, we proceed under the assumption (2.3), however we believe the result holds in greater generality, and notably in the $2 \mathrm{~d}$ case where $\partial \Omega$ is not flat in the neighborhood of $\Sigma_{D}$; see Remark 2.4. Let us mention that a quite similar problem is investigated from the theoretical viewpoint in [17], with stronger conclusions. Our first result in this direction is the following:

Theorem 4.2. Under assumption (2.3), the function $u_{\Omega, \varepsilon}$ converges to $u_{\Omega}$ strongly in $H^{1}(\Omega)$, and the following estimate holds:

$$
\left\|u_{\Omega, \varepsilon}-u_{\Omega}\right\|_{H^{1}(\Omega)} \leq C_{s} \varepsilon^{s}|| f \|_{L^{2}(\Omega)},
$$

for any $0<s<\frac{1}{4}$, where the constant $C_{s}$ depends on $s$.

Proof. The error $r_{\varepsilon}:=u_{\Omega, \varepsilon}-u_{\Omega}$ is the unique solution in $H^{1}(\Omega)$ to the system:

$$
\left\{\begin{array}{cl}
-\Delta r_{\varepsilon}=0 & \text { in } \Omega, \\
\frac{\partial r_{\varepsilon}}{\partial n}+h_{\varepsilon} r_{\varepsilon}=-\frac{\partial u_{\Omega}}{\partial n}-h_{\varepsilon} u_{\Omega} & \text { on } \partial \Omega,
\end{array}\right.
$$

which rewrites, under variational form:

$$
\forall v \in H^{1}(\Omega), \int_{\Omega} \nabla r_{\varepsilon} \cdot \nabla v d x+\int_{\partial \Omega} h_{\varepsilon} r_{\varepsilon} v d s=-\int_{\partial \Omega} \frac{\partial u_{\Omega}}{\partial n} v d s-\int_{\Gamma_{D} \cup \Gamma} h_{\varepsilon} u_{\Omega} v d s .
$$


Note that the above variational problem is well-posed, as follows from the Lax-Milgram lemma and the following Poincaré-like inequality (which is proved by the standard contradiction argument):

$$
\forall v \in H^{1}(\Omega),\|v\|_{H^{1}(\Omega)}^{2} \leq C\left(\int_{\Omega}|\nabla v|^{2} d x+\int_{\Gamma_{D}} v^{2} d s\right)
$$

here and throughout the proof, $C$ stands for a positive constant which is independent of $\varepsilon$. The estimate (4.14) is then obtained within two steps.

Step 1: We prove that $r_{\varepsilon}$ is bounded in $H^{1}(\Omega)$, uniformly with respect to $\varepsilon$.

To this end, we estimate the first term in the right-hand side of (4.16) as:

$$
\left|\int_{\partial \Omega} \frac{\partial u_{\Omega}}{\partial n} v d s\right| \leq C|| \frac{\partial u_{\Omega}}{\partial n}\left\|_{H^{-1 / 2}(\partial \Omega)}\right\| v \|_{H^{1}(\Omega)}
$$

where we have the control

$$
\left\|\frac{\partial u_{\Omega}}{\partial n}\right\|_{H^{-1 / 2}(\partial \Omega)} \leq C\|f\|_{L^{2}\left(\mathbb{R}^{d}\right)},
$$

as follows from the Green's formula (3.6) applied to the function $u_{\Omega}$ in $E\left(\Delta, L^{2}(\Omega)\right)$ (see (3.5)). We are thus left with the task of estimating the second term in the right-hand side of (4.16), that is, the integral:

$$
\int_{\Gamma_{D} \cup \Gamma} h_{\varepsilon} u_{\Omega} v d s=\int_{\Gamma} h_{\varepsilon} u_{\Omega} v d s
$$

To achieve this goal, recall that, since $u_{\Omega} \in H^{s}(\Omega)$ for $\frac{1}{2}<s<\frac{3}{2}$, and owing to the continuity of the trace $\left.u \mapsto u\right|_{\partial \Omega}$ from $H^{s}(\Omega)$ into $H^{s-\frac{1}{2}}(\partial \Omega)$, for $s>\frac{1}{2}$ (see e.g. [44], Th. 3.37), it comes that $u_{\Omega} \in H^{s-\frac{1}{2}}(\partial \Omega$ ), and in fact, using (2.2), that $u_{\Omega} \in \widetilde{H}^{s-\frac{1}{2}}\left(\Gamma_{N} \cup \Gamma\right)$. Using now the characterization (2.10) of the space $\widetilde{H}^{s-\frac{1}{2}}\left(\Gamma_{N} \cup \Gamma\right)$, it follows that for all $0<\sigma<1$, the function $\frac{1}{\rho^{\sigma}} u_{\Omega}$ belongs to $L^{2}(\Gamma)$, where we have introduced the weight $\rho(x):=\min \left(\left|x-s_{0}\right|,\left|x-s_{1}\right|\right)$.

Using this fact in combination with the Sobolev embedding from $H^{1 / 2}(\Gamma)$ into $L^{q}(\Gamma)$ for any $1 \leq q<\infty$ (see e.g. [1]), we get successively:

$$
\begin{aligned}
\left|\int_{\Gamma} h_{\varepsilon} u_{\Omega} v d s\right| & =\left|\int_{\Gamma} \rho^{\sigma} h_{\varepsilon} \frac{1}{\rho^{\sigma}} u_{\Omega} v d s\right| \\
& \leq\left(\int_{\Gamma} \rho^{p \sigma} h_{\varepsilon}^{p} d s\right)^{\frac{1}{p}}\left(\int_{\Gamma} \frac{1}{\rho^{2 \sigma}} u_{\Omega}^{2} d s\right)^{\frac{1}{2}}\left(\int_{\Gamma} v^{q} d s\right)^{\frac{1}{q}} \\
& \leq\left(\int_{\Gamma} \rho^{p \sigma} h_{\varepsilon}^{p} d s\right)^{\frac{1}{p}}\left\|u_{\Omega}\right\|_{\widetilde{H}^{\sigma}\left(\Gamma_{N} \cup \Gamma\right)}\|v\|_{L^{q}(\Gamma)}, \\
& \leq C\left(\int_{\Gamma} \rho^{p \sigma} h_{\varepsilon}^{p} d s\right)^{\frac{1}{p}}\left\|u_{\Omega}\right\|_{\widetilde{H}^{\sigma}\left(\Gamma_{N} \cup \Gamma\right)}\|v\|_{H^{1}(\Omega)},
\end{aligned}
$$

for any $p>2$ (the constant $C$ depends on $p$ ), where we have used Hölder's inequality with $\frac{1}{2}+\frac{1}{p}+\frac{1}{q}=1$ to pass from the first line to the second one.

To proceed further, let us decompose $\Gamma$ as

$$
\Gamma=\Gamma_{\varepsilon} \cup \overline{U_{0}} \cup \overline{U_{1}}, \text { where } U_{i}:=\left\{x \in \Gamma,\left|x-s_{i}\right|<\varepsilon\right\}, \text { and } \Gamma_{\varepsilon}:=\{x \in \Gamma, \rho(x)>\varepsilon\} .
$$

Taking advantage of the structure (4.3) of $h_{\varepsilon}$, the first integral in the right-hand side of (4.19) is of the form

$$
\begin{aligned}
\int_{\Gamma} \rho^{p \sigma} h_{\varepsilon}^{p} d s & =\int_{U_{1}} \rho^{p \sigma} h_{\varepsilon}^{p} d s+\int_{U_{2}} \rho^{p \sigma} h_{\varepsilon}^{p} d s+\int_{\Gamma_{\varepsilon}} \rho^{p \sigma} h_{\varepsilon}^{p} d s \\
& \leq \frac{C}{\varepsilon^{p}} \int_{0}^{\varepsilon} t^{p \sigma} h\left(\frac{t}{\varepsilon}\right)^{p} d t \\
& \leq \frac{C \varepsilon^{p \sigma+1}}{\varepsilon^{p}} \int_{0}^{1} t^{p \sigma} h(t)^{p} d t \\
& \leq C \varepsilon^{p(\sigma-1)+1} .
\end{aligned}
$$


Therefore,

$$
\left(\int_{\Gamma} \rho^{p \sigma} h_{\varepsilon}^{p} d s\right)^{\frac{1}{p}} \leq C \varepsilon^{\sigma-1+\frac{1}{p}} ;
$$

now, choosing $p>2$ and $\sigma<1$ adequately and using (2.11), (2.13) and (4.19), we have proved that, for all $s<\frac{1}{2}$, there exists a constant $C_{s}$ :

$$
\left|\int_{\Gamma} h_{\varepsilon} u_{\Omega} v d s\right| \leq C_{s} \varepsilon^{s}\|f\|_{L^{2}\left(\mathbb{R}^{d}\right)}\|v\|_{H^{1}(\Omega)} .
$$

Eventually, taking $v=r_{\varepsilon}$ as a test function in (4.16), we obtain the standard a priori estimate for $r_{\varepsilon}$ :

$$
\int_{\Omega}\left|\nabla r_{\varepsilon}\right|^{2} d x+\int_{\partial \Omega} h_{\varepsilon} r_{\varepsilon}^{2} d s=-\int_{\partial \Omega} \frac{\partial u_{\Omega}}{\partial n} r_{\varepsilon} d s-\int_{\Gamma} h_{\varepsilon} u_{\Omega} r_{\varepsilon} d s
$$

Combining (4.21) with the estimates (4.18) and (4.20), the Poincaré inequality (4.17) and the fact that $h_{\varepsilon} \geq 1$ on $\Gamma_{D}$ (see (4.4)), it follows that there exists a constant $C$, which does not depend on $\varepsilon$, such that:

$$
\left\|r_{\varepsilon}\right\|_{H^{1}(\Omega)} \leq C\|f\|_{L^{2}\left(\mathbb{R}^{d}\right)} .
$$

Step 2: We now turn to the proof of (4.14) so to speak.

Multiplying both sides of (4.21) by $\varepsilon$ and using (4.4), we obtain:

$$
\begin{aligned}
\left\|r_{\varepsilon}\right\|_{L^{2}\left(\Gamma_{D}\right)}^{2} & \leq C \varepsilon \int_{\Gamma_{D}} h_{\varepsilon} r_{\varepsilon}^{2} d s, \\
& \leq C \varepsilon\left(\int_{\Omega}\left|\nabla r_{\varepsilon}\right|^{2} d x+\int_{\partial \Omega} h_{\varepsilon} r_{\varepsilon}^{2} d s\right) \\
& \leq C \varepsilon\left|\int_{\partial \Omega}^{2} \frac{\partial u_{\Omega}}{\partial n} r_{\varepsilon} d s\right|+C \varepsilon\left|\int_{\Gamma} h_{\varepsilon} u_{\Omega} r_{\varepsilon} d s\right|, \\
& \leq C \varepsilon\|f\|_{L^{2}\left(\mathbb{R}^{d}\right)}^{2},
\end{aligned}
$$

where we have used the estimate (4.20) with $v=r_{\varepsilon}$ and the bound (4.22) over $r_{\varepsilon}$.

Interpolating between (4.22) and (4.23) (see for instance [40]), for all $0 \leq s \leq \frac{1}{2}, s=(1-t) 0+\frac{1}{2} t$, there exists a constant $C_{s}$ such that:

$$
\left\|r_{\varepsilon}\right\|_{H^{s}\left(\Gamma_{D}\right)} \leq C_{s}\left\|r_{\varepsilon}\right\|_{L^{2}\left(\Gamma_{D}\right)}^{1-t}\left\|r_{\varepsilon}\right\|_{H^{\frac{1}{2}\left(\Gamma_{D}\right)}}^{t} \leq C_{s} \varepsilon^{\frac{1}{2}-s}\|f\|_{L^{2}\left(\mathbb{R}^{d}\right)} .
$$

Now, since $u_{\Omega} \in H^{s}(\Omega)$ for $\frac{1}{2}<s<\frac{3}{2}$, it comes that $\frac{\partial u_{\Omega}}{\partial n} \in H^{s-\frac{3}{2}}(\partial \Omega)$ (see [16], Lemma 4.3), and so

$$
\left|\int_{\Gamma_{D}} \frac{\partial u_{\Omega}}{\partial n} r_{\varepsilon} d s\right| \leq\left\|\frac{\partial u_{\Omega}}{\partial n}\right\|_{H^{s-\frac{3}{2}}\left(\Gamma_{D}\right)}\left\|r_{\varepsilon}\right\|_{H^{\frac{3}{2}-s}\left(\Gamma_{D}\right)} \leq C_{s} \varepsilon^{s-1}\|f\|_{L^{2}\left(\mathbb{R}^{d}\right)}^{2},
$$

for all $1<s<\frac{3}{2}$. Returning to (4.21) and using (4.17), (4.20) and (4.24), we now see that, for any $s<\frac{1}{2}$ and $\sigma<\frac{1}{2}$, there exists a constant $C>0$ (depending on $s$ and $\sigma$ ) such that:

$$
\begin{aligned}
\left\|r_{\varepsilon}\right\|_{H^{1}(\Omega)}^{2} & \leq C\left(\int_{\Omega}\left|\nabla r_{\varepsilon}\right|^{2} d s+\int_{\Gamma_{D}} r_{\varepsilon}^{2} d s\right) \\
& \leq C\left(\varepsilon^{s}\|f\|_{L^{2}\left(\mathbb{R}^{d}\right)}^{2}+\varepsilon^{\sigma}\|f\|_{L^{2}\left(\mathbb{R}^{d}\right)}\left\|r_{\varepsilon}\right\|_{H^{1}(\Omega)}\right),
\end{aligned}
$$

Hence (4.14) holds, and this terminates the proof.

As a straightforward consequence of Theorem 4.2, we obtain:

Corollary 4.1. Under assumption (2.3), for any given admissible shape $\Omega \in \mathcal{U}_{\mathrm{DN}}$, the approximate shape functional $J_{\varepsilon}(\Omega)$ converges to its exact counterpart $J(\Omega)$.

Let us now turn to the convergence of the derivative of $J_{\varepsilon}(\Omega)$.

Theorem 4.3. Under Assumption (2.3), for a given admissible shape $\Omega \in \mathcal{U}_{\mathrm{DN}}$, the approximate shape derivative $J_{\varepsilon}^{\prime}(\Omega)$ converges to its exact counterpart $J^{\prime}(\Omega)$ in the sense that:

$$
\sup _{\substack{\theta \in \Theta_{\mathrm{DN}},\|\theta\|_{\mathrm{DN}} \leq 1}}\left|J_{\varepsilon}^{\prime}(\Omega)(\theta)-J^{\prime}(\Omega)(\theta)\right|=0
$$


Proof. We rely on the volumetric expressions (3.13) and (4.7) of the shape derivatives $J^{\prime}(\Omega)(\theta)$ and $J_{\varepsilon}^{\prime}(\Omega)(\theta)$. In our context where (2.3) is satisfied, the boundary $\partial \Omega$ is flat in the neighborhood of $\Sigma_{D}=\left\{s_{0}, s_{1}\right\}$; hence, for $\varepsilon>0$ small enough, the second fundamental form of $\partial \Omega$ vanishes where $h_{\varepsilon}>0$, and the normal vectors $n_{\Sigma_{D}}\left(s_{0}\right), n_{\Sigma_{D}}\left(s_{1}\right)$ to $\Sigma_{D}$ coincide with the tangent vectors $\pm \tau\left(s_{0}\right)$ and $\pm \tau\left(s_{1}\right)$ to $\partial \Omega$. Then, the approximate shape derivative $J_{\varepsilon}^{\prime}(\Omega)$ supplied by Proposition 4.3 simply boils down to:

$$
\begin{gathered}
J^{\prime}(\Omega)(\theta)=\int_{\partial \Omega}\left(j\left(u_{\Omega, \varepsilon}\right)-f p_{\Omega, \varepsilon}\right) \theta \cdot n d s-\int_{\Omega} j^{\prime}\left(u_{\Omega, \varepsilon}\right) \nabla u_{\Omega, \varepsilon} \cdot \theta d x+\int_{\Omega}\left(\operatorname{div} \theta \mathrm{I}-\nabla \theta-\nabla \theta^{T}\right) \nabla u_{\Omega, \varepsilon} \cdot \nabla p_{\Omega, \varepsilon} d x \\
+\int_{\Gamma \cup \Gamma_{D}} \operatorname{div}_{\partial \Omega} \theta h_{\varepsilon} u_{\Omega, \varepsilon} p_{\Omega, \varepsilon} d s+\frac{1}{\varepsilon^{2}} \int_{\Gamma \cup \Gamma_{D}} h^{\prime}\left(\frac{x-s_{0}}{\varepsilon}\right)\left(\theta(x)-\theta\left(s_{0}\right)\right) \cdot n_{\Sigma_{D}}\left(s_{0}\right) u_{\Omega, \varepsilon} p_{\Omega, \varepsilon} d s(x) \\
+\frac{1}{\varepsilon^{2}} \int_{\Gamma \cup \Gamma_{D}} h^{\prime}\left(\frac{x-s_{1}}{\varepsilon}\right)\left(\theta(x)-\theta\left(s_{1}\right)\right) \cdot n_{\Sigma_{D}}\left(s_{1}\right) u_{\Omega, \varepsilon} p_{\Omega, \varepsilon} d s(x)+\int_{\Omega} f \nabla p_{\Omega, \varepsilon} \cdot \theta d x .
\end{gathered}
$$

Given the expression (3.13) of the exact shape derivative $J^{\prime}(\Omega)$, and in light of Theorem 4.2, it is obviously enough to show that the three integrals

$$
I_{1}(\theta):=\int_{\Gamma \cup \Gamma_{D}} \operatorname{div}_{\Gamma} \theta h_{\varepsilon} u_{\Omega, \varepsilon} p_{\Omega, \varepsilon} d s, I_{2}(\theta):=\frac{1}{\varepsilon^{2}} \int_{\Gamma \cup \Gamma_{D}} h^{\prime}\left(\frac{x-s_{0}}{\varepsilon}\right)\left(\theta(x)-\theta\left(s_{0}\right)\right) \cdot \tau\left(s_{0}\right) u_{\Omega, \varepsilon} p_{\Omega, \varepsilon} d s(x),
$$

and

$$
I_{3}(\theta):=\frac{1}{\varepsilon^{2}} \int_{\Gamma \cup \Gamma_{D}} h^{\prime}\left(\frac{x-s_{1}}{\varepsilon}\right)\left(\theta(x)-\theta\left(s_{1}\right)\right) \cdot \tau\left(s_{1}\right) u_{\Omega, \varepsilon} p_{\Omega, \varepsilon} d s(x)
$$

converge to 0 as $\varepsilon \rightarrow 0$, uniformly with respect to $\theta$ when $\|\theta\|_{\Theta_{\mathrm{DN}}} \leq 1$.

As far as the integral $I_{1}(\theta)$ is concerned, Theorem 4.2 and the facts that $-\Delta u_{\varepsilon}=-\Delta u_{\Omega}=f$ imply that

$$
\frac{\partial u_{\Omega, \varepsilon}}{\partial n} \rightarrow \frac{\partial u_{\Omega}}{\partial n} \text { in } H^{-1 / 2}(\partial \Omega), \text { and } u_{\Omega, \varepsilon} \rightarrow u_{\Omega} \text { in } H^{1 / 2}(\partial \Omega) \text { as } \varepsilon \rightarrow 0 ;
$$

similar convergence results hold about $p_{\Omega, \varepsilon}$ and $p_{\Omega}$. Therefore,

$$
\int_{\Gamma \cup \Gamma_{D}} \operatorname{div}_{\Gamma} \theta h_{\Omega, \varepsilon} u_{\Omega, \varepsilon} p_{\Omega, \varepsilon} d s \stackrel{\varepsilon \rightarrow 0}{\longrightarrow}-\int_{\Gamma \cup \Gamma_{D}} \operatorname{div}_{\Gamma} \theta \frac{\partial u_{\Omega}}{\partial n} p_{\Omega} d s
$$

where the last integral may be decomposed as

$$
\int_{\Gamma \cup \Gamma_{D}} \operatorname{div}_{\partial \Omega} \theta \frac{\partial u_{\Omega}}{\partial n} p_{\Omega} d s=\int_{\Gamma_{D}} \operatorname{div}_{\partial \Omega} \theta \frac{\partial u_{\Omega}}{\partial n} p_{\Omega} d s+\int_{\Gamma} \operatorname{div}_{\partial \Omega} \theta \frac{\partial u_{\Omega}}{\partial n} p_{\Omega} d s=0
$$

as follows from the boundary conditions satisfied by $u_{\Omega}$ and $p_{\Omega}$. This convergence is easily seen to be uniform with respect to $\theta \in \Theta_{\mathrm{DN}},\|\theta\|_{\Theta_{\mathrm{DN}}} \leq 1$.

Let us now turn to the treatment of $I_{2}(\theta)$, that of $I_{3}(\theta)$ being on all points identical. We assume for notation simplicity that $s_{0}=0$, and again, we identify the neighborhood of $s_{0}$ in $\partial \Omega$ (which is a horizontal line) with a subset of the real line $\mathbb{R}$. The key remark in the analysis of $I_{2}(\theta)$ is that there exists a vector field $\widetilde{\theta}(x)$ vanishing identically on $\Gamma_{N}$ such that $(\theta(x)-\theta(0)) \cdot \tau(0)=x \cdot \tilde{\theta}(x)$, as is easily seen from a Taylor expansion at 0 . This will allow to improve the available convergence rates of $u_{\Omega, \varepsilon}$ and $p_{\Omega, \varepsilon}$ in the integrand of $I_{2}(\theta)$. More precisely, using integration by parts on the boundary $\partial \Omega, I_{2}(\theta)$ rewrites:

$$
\begin{aligned}
I_{2}(\theta) & =\int_{\Gamma \cup \Gamma_{D}} \frac{\partial h_{\varepsilon}}{\partial \tau} x \cdot \tilde{\theta}(x) u_{\Omega, \varepsilon} p_{\Omega, \varepsilon} d s \\
& =-\int_{\Gamma \cup \Gamma_{D}} h_{\varepsilon} \frac{\partial}{\partial \tau}\left(x \cdot \tilde{\theta}(x) u_{\Omega, \varepsilon} p_{\Omega, \varepsilon}\right) d s, \\
& =-\int_{\Gamma \cup \Gamma_{D}} h_{\varepsilon} \tilde{\theta}(x) \cdot\left(x \frac{\partial u_{\Omega, \varepsilon}}{\partial \tau} p_{\Omega, \varepsilon}+x \frac{\partial p_{\Omega, \varepsilon}}{\partial \tau} u_{\Omega, \varepsilon}\right) d s-\int_{\Gamma \cup \Gamma_{D}} h_{\varepsilon} u_{\Omega, \varepsilon} p_{\Omega, \varepsilon} \frac{\partial(x \cdot \tilde{\theta}(x))}{\partial \tau} d s \\
& =-\int_{\Gamma \cup \Gamma_{D}} h_{\varepsilon} \tilde{\theta}(x) \cdot\left(x \frac{\partial u_{\Omega, \varepsilon}}{\partial \tau} p_{\Omega, \varepsilon}+x \frac{\partial p_{\Omega, \varepsilon}}{\partial \tau} u_{\Omega, \varepsilon}\right) d s+R_{\varepsilon}(\theta),
\end{aligned}
$$

where $R_{\varepsilon}(\theta)$ is a remainder (possibly changing from one line to the next) gathering several integrals which are proved to converge to 0 as $\varepsilon \rightarrow 0$, uniformly with respect to $\theta$ when $\|\theta\|_{\Theta_{\mathrm{DN}}} \leq 1$ owing to similar calculations 
to those involved in the above proof of convergence of $I_{1}(\theta)$ (see (4.26)). Then, using the boundary conditions satisfied by $u_{\Omega, \varepsilon}$ and $p_{\Omega, \varepsilon}$,

$$
\begin{aligned}
I_{2}(\theta) & =\int_{\Gamma \cup \Gamma_{D}}\left(\rho(x) \frac{\partial u_{\Omega, \varepsilon}}{\partial \tau} \frac{\partial p_{\Omega, \varepsilon}}{\partial n}+\rho(x) \frac{\partial p_{\Omega, \varepsilon}}{\partial \tau} \frac{\partial u_{\Omega, \varepsilon}}{\partial n}\right)\left(\frac{x}{\rho(x)} \cdot \tilde{\theta}(x)\right) d s+R_{\varepsilon}(\theta), \\
& =\int_{\Gamma \cup \Gamma_{D}}\left(\frac{\partial\left(\rho u_{\Omega, \varepsilon}\right)}{\partial \tau} \frac{\partial p_{\Omega, \varepsilon}}{\partial n}+\frac{\partial\left(\rho p_{\Omega, \varepsilon}\right)}{\partial \tau} \frac{\partial u_{\Omega, \varepsilon}}{\partial n}\right)\left(\frac{x}{\rho(x)} \cdot \tilde{\theta}(x)\right) d s+R_{\varepsilon}(\theta),
\end{aligned}
$$

where we have posed $\rho(x)=|x|$ and the same calculations as in (4.26) have been used.

At this point, we know from Theorem 4.2 that $\frac{\partial u_{\Omega, \varepsilon}}{\partial n}$ (resp. $\frac{\partial p_{\Omega, \varepsilon}}{\partial n}$ ) converges to $\frac{\partial u_{\Omega}}{\partial n}$ (resp. $\frac{\partial p_{\Omega}}{\partial n}$ ) in $H^{-1 / 2}(\partial \Omega)$. Hence, the proof of the convergence of $I_{2}(\theta)$, and thereby that of Theorem 4.3, follows from the following results:

$$
\frac{\partial\left(\rho u_{\Omega, \varepsilon}\right)}{\partial \tau} \stackrel{\varepsilon \rightarrow 0}{\longrightarrow} \frac{\partial\left(\rho u_{\Omega}\right)}{\partial \tau} \text { in } H^{1}(\Omega), \text { and } \frac{\partial\left(\rho p_{\Omega, \varepsilon}\right)}{\partial \tau} \stackrel{\varepsilon \rightarrow 0}{\longrightarrow} \frac{\partial\left(\rho p_{\Omega}\right)}{\partial \tau} \text { in } H^{1}(\Omega),
$$

where $\tau$ stands for any smooth extension to the whole $\Omega$ of the tangent vector $\tau$ to $\partial \Omega$; see Appendix A. We now sketch the proof of this last statement focusing on the case of $u_{\Omega, \varepsilon}$; the counterpart result as regards $p_{\Omega, \varepsilon}$ being proved in a similar fashion.

The convergence (4.27) actually follows from exactly the same arguments as that in the proof of (4.14). At first, using the representation of Proposition 2.1 (or more exactly a higher-order avatar of it, see Remark 2.3), observe that the function $\rho u_{\Omega}$ belongs to $H^{s}(\Omega)$ for all $0 \leq s<\frac{5}{2}$. Letting the notation $r_{\varepsilon}:=u_{\Omega, \varepsilon}-u_{\Omega}$, and using test functions of the form $\rho(x) v \in H^{1}(\Omega)$ for $v \in H^{1}(\Omega)$ inside the variational formulation (4.16) of $r_{\varepsilon}$, we see that $\rho r_{\varepsilon}$ satisfies:

$$
\begin{aligned}
\forall v \in H^{1}(\Omega), \int_{\Omega} \nabla\left(\rho r_{\varepsilon}\right) \cdot \nabla v d x+ & \int_{\Gamma \cup \Gamma_{D}} h_{\varepsilon} \rho r_{\varepsilon} v d s= \\
& \quad-\int_{\Gamma} h_{\varepsilon} \rho u_{\Omega} v d s-\int_{\Gamma_{D}} \frac{\partial u_{\Omega}}{\partial n} \rho v d s-\int_{\Omega} \nabla \rho \cdot\left(v \nabla r_{\varepsilon}-r_{\varepsilon} \nabla v\right) d x .
\end{aligned}
$$

Now using test functions of the form $\frac{\partial v}{\partial \tau}, v \in H^{1}(\Omega)$ in (4.28), then integrating by parts yields the following variational formulation for $q_{\varepsilon}:=\frac{\partial\left(\rho r_{\varepsilon}\right)}{\partial \tau}$ :

$$
\begin{aligned}
\forall v \in H^{1}(\Omega),-\int_{\Omega} \nabla q_{\varepsilon} \cdot \nabla v d x-\int_{\Gamma \cup \Gamma_{D}} h_{\varepsilon} q_{\varepsilon} v d s= & \int_{\partial \Omega} \frac{\partial h_{\varepsilon}}{\partial \tau} \rho r_{\varepsilon} v d s+\int_{\Gamma} \frac{\partial}{\partial \tau}\left(h_{\varepsilon} \rho(x) u_{\Omega}\right) v d s \\
& +\int_{\Gamma_{D}} \frac{\partial}{\partial \tau}\left(\rho \frac{\partial u_{\Omega}}{\partial n}\right) v d s+\left\langle F_{\varepsilon}, v\right\rangle_{H^{1}(\Omega)^{*}, H^{1}(\Omega)},
\end{aligned}
$$

where the remainder $F_{\varepsilon}$ is a sequence of linear forms in the dual $H^{1}(\Omega)^{*}$ of $H^{1}(\Omega)$ which converges to 0 in the strong dual topology.

Finally, using the result of Theorem 4.2, together with very similar calculations than those involved in its proof, the desired result (4.27) follows, which concludes the proof.

\section{NUMERICAL ILLUSTRATIONS}

In this final section we present several numerical illustrations of the previous mathematical developments. The examples discussed below take place in situations which are variations of the basic model introduced in Section 2.1. The notations may differ slightly depending on the context at stake, and so do the involved numerical methods; the precise description of the considered setting is adapted to each particular case.

One common feature of our examples is that all of the simulations are done using the finite element method in FreeFem++ [32] with Lagrange $\mathbb{P}_{1}$ finite elements. When it comes to operations related to the level set method we used our own C++ implementation interfaced within FreeFem++. Finally, all computations were done on a basic laptop.

We start in Section 5.1 with a fairly simple academic example which aims to validate the approximation procedure of Section 4 for optimization problems featuring the transition between regions supporting homogeneous Dirichlet and Neumann boundary conditions. We then turn in Sections 5.2 and 5.3 to more realistic applications in the context of the linearized elasticity equations. Specifically, Section 5.3 deals with problems 
where both the shape and the region of its boundary supporting Dirichlet boundary conditions are subject to optimization.

\subsection{An academic, validation example}

We start with a toy example, meant to assess the validity of the approximation process of Section 4 for the optimization of the transition $\Sigma_{D}$ between regions of the shape $\Omega$ bearing homogeneous Dirichlet and Neumann boundary conditions.

We consider a rectangular domain $\Omega=(-1,1) \times\left(-\frac{1}{2}, \frac{1}{2}\right)$, as depicted on Fig. 4 (left), which is filled with a material with inhomogeneous conductivity $a \in L^{\infty}(\Omega)$ satisfying the conditions

$$
m \leq a(x) \leq M \text { a.e. } x \in \Omega,
$$

for some constants $0<m \leq M$. To be quite precise, in the present application, $a(x)$ takes the values 1 inside a (fixed) subset $K \subset \Omega$, and 100 outside $K$. A constant, unit body source is acting inside $\Omega$, and the boundary $\partial \Omega$ is divided into two distinct regions $\Gamma_{D}$ and $\Gamma$ supporting respectively homogeneous Dirichlet and Neumann boundary conditions. The region $\Gamma_{D}$ is parametrized by two real parameters $\alpha$, $\beta$, and is thenceforth denoted by $\Gamma_{\alpha, \beta}$; it is of the form:

$$
\Gamma_{\alpha, \beta}=\left\{\left(\alpha+t,-\frac{1}{2}\right), t \in(-w, w)\right\} \cup\left\{\left(\beta+t, \frac{1}{2}\right), t \in(-w, w)\right\} ;
$$

in other terms, $\Gamma_{\alpha, \beta}$ is the reunion of two intervals with fixed width $w>0$, which are respectively centered at the points $\left(\alpha,-\frac{1}{2}\right)$ and $\left(\beta, \frac{1}{2}\right) \in \partial \Omega$, where $\alpha, \beta \in(-1+w, 1-w)$. In this situation, the voltage potential $u_{\alpha, \beta}$ inside $\Omega$ arises as the unique solution in $H_{\Gamma_{\alpha, \beta}}^{1}(\Omega)$ to the conductivity equation:

$$
\left\{\begin{array}{cl}
-\operatorname{div}\left(a(x) \nabla u_{\alpha, \beta}\right)=1 & \text { in } \Omega, \\
u_{\alpha, \beta}=0 & \text { on } \Gamma_{\alpha, \beta}, \\
\frac{\partial u_{\alpha, \beta}}{\partial n}=0 & \text { on } \Gamma .
\end{array}\right.
$$

Our purpose is to optimize the position $(\alpha, \beta)$ of the two intervals defining $\Gamma_{\alpha, \beta}$ so as to minimize the average potential inside the region $K$; that is, we considered the following optimization problem of two real parameters:

$$
\inf _{(\alpha, \beta) \in(-1+w, 1-w)^{2}} J(\alpha, \beta), \text { where } J(\alpha, \beta):=\int_{K} u_{\alpha, \beta} d x .
$$

We first perform a brute-force calculation of all the possible values $J(\Omega)$ when the couple $(\alpha, \beta)$ runs through the set $(-1+w, 1-w)^{2}$. The results are reported on Fig. 4 . In particular, it is visible that $J(\alpha, \beta)$ possesses two local minima, near the points $(-0.5,0.5)$ and $(0.5,-0.5)$, respectively.
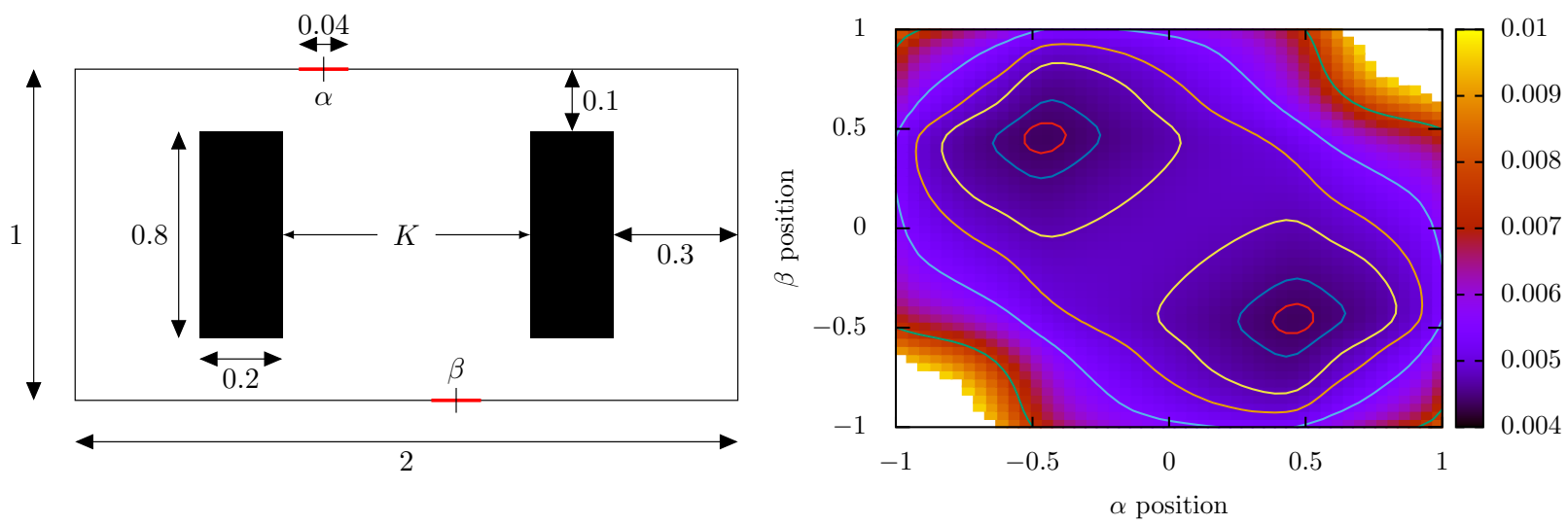

Figure 4. (Left) Setting of the validation example of Section 5.1; (right) value of the objective function for all admissible positions of the boundary $\Gamma_{D}$, parametrized by $\alpha$ and $\beta$. 
According to the methodology developed in Section 4, we now approximate the problem (5.3) by that

$$
\inf _{(\alpha, \beta) \in(-1+w, 1-w)^{2}} J_{\varepsilon}(\alpha, \beta), \text { where } J_{\varepsilon}(\alpha, \beta):=\int_{K} u_{\alpha, \beta, \varepsilon} d x
$$

featuring the solution $u_{\alpha, \beta, \varepsilon} \in H^{1}(\Omega)$ to the following smoothed counterpart of (5.2):

$$
\left\{\begin{array}{cl}
-\operatorname{div}\left(a(x) \nabla u_{\alpha, \beta, \varepsilon}\right)=1 & \text { in } \Omega, \\
\frac{\partial u_{\alpha, \beta, \varepsilon}}{\partial n}+h_{\varepsilon} u_{\alpha, \beta, \varepsilon}=0 & \text { on } \partial \Omega .
\end{array}\right.
$$

In (5.5), the interpolation profile $h_{\varepsilon}$ is constructed as in (4.3), in which the small parameter $\varepsilon$ is chosen of the order of the mesh size.

The partial derivatives of $J_{\varepsilon}(\alpha, \beta)$ are easily calculated thanks to Proposition 4.3, taking advantage of the particularly simple form (5.1) of the optimized region $\Gamma_{D}$ of $\partial \Omega$; for instance, one has:

$$
\frac{\partial J_{\varepsilon}}{\partial \alpha}(\alpha, \beta)=-\frac{1}{\varepsilon^{2}} \int_{L_{\alpha}^{+}} h^{\prime}\left(\frac{t-\alpha-w}{\varepsilon}\right) u_{\alpha, \beta, \varepsilon} p_{\alpha, \beta, \varepsilon} d t+\frac{1}{\varepsilon^{2}} \int_{L_{\alpha}^{-}} h^{\prime}\left(\frac{-t+\alpha-w}{\varepsilon}\right) u_{\alpha, \beta, \varepsilon} p_{\alpha, \beta, \varepsilon} d t,
$$

where $L_{\alpha}^{+}, L_{\alpha}^{-}$are the one-dimensional sets defined by $L_{\alpha}^{+}=\left\{\left(t,-\frac{1}{2}\right), t>\alpha\right\}, L_{\alpha}^{-}=\left\{\left(t,-\frac{1}{2}\right), t<\alpha\right\}$ (with a little abuse of notations, $L_{\alpha}^{ \pm}$are identified to the corresponding subsets of the real line). In the above expression, the adjoint state $p_{\alpha, \beta, \varepsilon}$ is the unique solution in $H^{1}(\Omega)$ to the problem:

$$
\left\{\begin{array}{cl}
-\operatorname{div}\left(a(x) \nabla p_{\alpha, \beta, \varepsilon}\right)=-\mathbb{1}_{K} & \text { in } \Omega, \\
\frac{\partial p_{\alpha, \beta, \varepsilon}}{\partial n}+h_{\varepsilon} p_{\alpha, \beta, \varepsilon}=0 & \text { on } \partial \Omega,
\end{array}\right.
$$

where $\mathbb{1}_{K}$ is the characteristic function of $K$. A similar expression holds for the partial derivative $\frac{\partial J_{\varepsilon}}{\partial \beta}$.

We solve the approximate problem (5.4) by means of a standard gradient algorithm, starting from several different initial configurations $(\alpha, \beta)$ as regards the two connected components of $\Gamma_{\alpha, \beta}$. The results are reported in Fig. 5: in particular, depending on the initialization, the boundary $\Gamma_{\alpha, \beta}$ smoothly converges to an optimized position which matches the two local minima of the exact functional $J(\alpha, \beta)$ (see again Fig. 4, (right)). This indicates a good agreement between the exact optimization problem (5.3) and its approximation (5.4), and notably between the exact and approximate derivatives of the optimized criterion.

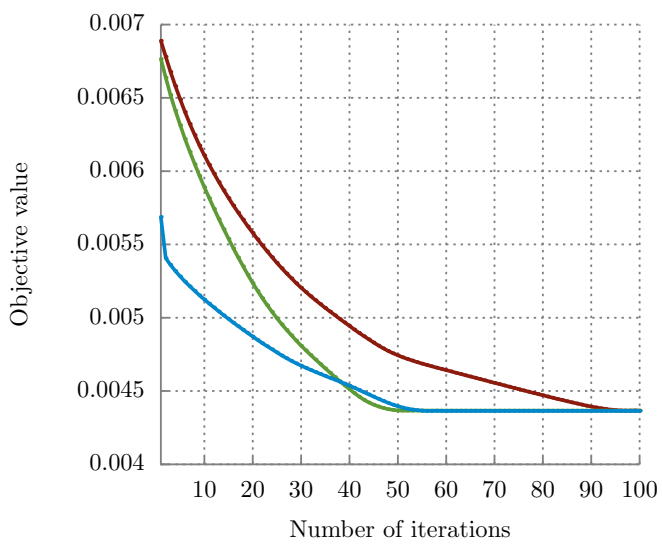

(A) Convergence history of the objective function

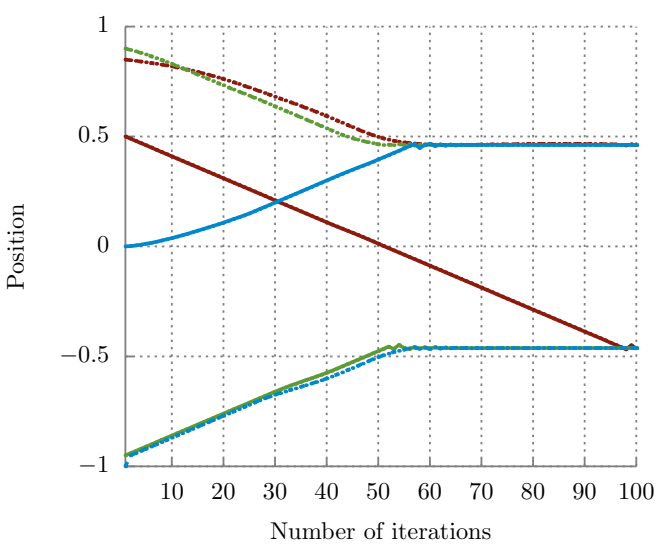

(в) Successive values of $\alpha$ (solid lines) and $\beta$ (dashed lines)

FiguRE 5. Histories of (left) the objective function $J_{\varepsilon}(\alpha, \beta)$ and (right) the locations $\alpha, \beta$ of the components of the optimized boundary $\Gamma_{\alpha, \beta}$ in the validation example of Section 5.1. Three experiments are performed, with different initial values $(\alpha, \beta)$ (associated to red, green and blue lines). 


\subsection{Optimization of the repartition of clamps and locators on the boundary of an elastic structure}

Our second example deals with the application of the results of Sections 3 and 4 to the problem of optimal repartition of clamps and locators on an elastic structure; see [11], Chap. 9, for a presentation of the physical context and $[36,41,50]$ for optimization studies conducted in this context.

\subsubsection{Description of the physical setting and of the optimization problem}

In this example, $\Omega$ stands for a three-dimensional rectangular beam with size $4 \times 1 \times 1$, filled with a linearly elastic material, whose Hooke's law $A$ is defined by, for any symmetric matrix $e$ with size $3 \times 3$ :

$$
A e=2 \mu e+\lambda \operatorname{tr}(e),
$$

where $\lambda, \mu$ are the Lamé parameters of the material; in our context

$$
\lambda=\frac{E \nu}{(1+\nu)(1-2 \nu)}, \quad \mu=\frac{E}{1+\nu}
$$

with $E=100, \nu=0.3$. During its construction, $\Omega$ receives the vertical load $g_{\text {tool }}=(0,0,-1)$ from the manufacturing tool, which is applied on the upper side $\Gamma_{T}$ of its boundary. So that the structure do not move under this effort, a clamping-locator system is used: $\Omega$ is attached on a subregion $\Gamma_{D}$ of the left-hand side $\Lambda_{D}$ of $\partial \Omega$ (locator), while it receives a prescribed load $g=(0,-1,0)$ on another region $\Gamma_{N}$ of the right-hand side $\Lambda_{N} \subset \partial \Omega$ (clamping); the latter is exerted by an external mechanical device pressing against the structure; see Fig. 6 for a sketch of the situation.

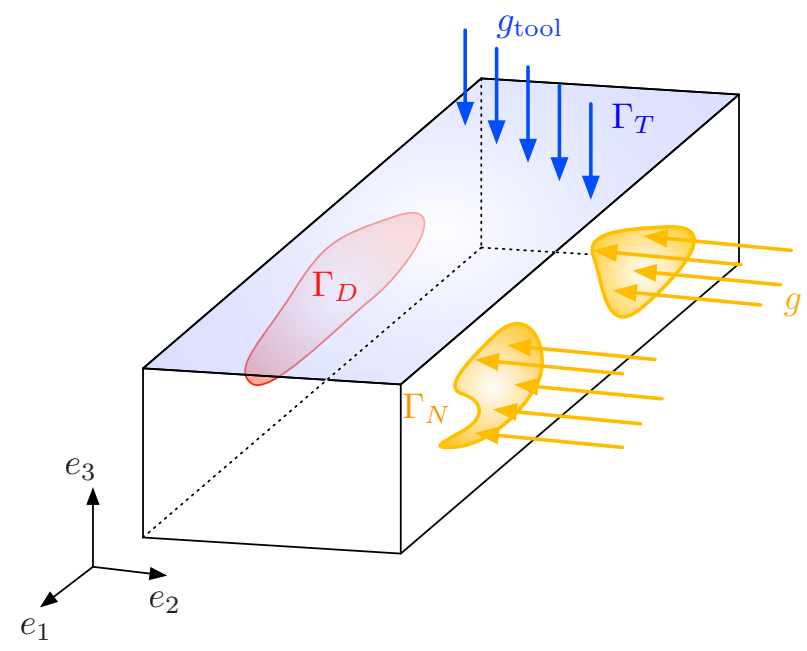

Figure 6. Setting of the example of Section 5.2 about the optimal repartition of clamps and locators on the boundary of an elastic structure.

In this context, the displacement of $\Omega$ is the unique solution $u_{\Gamma_{D}, \Gamma_{N}} \in H_{\Gamma_{D}}^{1}(\Omega)^{3}$ to the following linear elasticity system:

$$
\left\{\begin{array}{cl}
-\operatorname{div}\left(A e\left(u_{\Gamma_{D}, \Gamma_{N}}\right)=0\right. & \text { in } \Omega, \\
u_{\Gamma_{D}, \Gamma_{N}}=0 & \text { on } \Gamma_{D}, \\
A e\left(u_{\Gamma_{D}, \Gamma_{N}}\right) n=g_{\text {tool }} & \text { on } \Gamma_{T}, \\
A e\left(u_{\Gamma_{D}, \Gamma_{N}}\right) n=g & \text { on } \Gamma_{N}, \\
A e\left(u_{\Gamma_{D}, \Gamma_{N}}\right) n=0 & \text { on } \Gamma,
\end{array}\right.
$$

where $e(u):=\frac{1}{2}\left(\nabla u+\nabla u^{T}\right)$ is the strain tensor associated to a vector field $u: \Omega \rightarrow \mathbb{R}^{3}$.

Our aim is to optimize the positions $\Gamma_{N}$ and $\Gamma_{D}$ of clamps and locators on the surface of the structure $\Omega$, whose shape itself is not subject to optimization, so that the displacement of $\Omega$ under the action of $g_{\text {tool }}$ 
be minimal. We also add constraints on the size of the regions $\Gamma_{N}, \Gamma_{D}$ and on the perimeter of $\Gamma_{D}$ via fixed penalizations of the objective function. More precisely, we consider the optimization problem:

$$
\inf _{\substack{\Gamma_{D} \subset \Lambda_{D} \\ \Gamma_{N} \subset \Lambda_{N}}} J\left(\Gamma_{D}, \Gamma_{N}\right) \text {, where } J\left(\Gamma_{D}, \Gamma_{N}\right)=\int_{\Omega}\left|u_{\Gamma_{D}, \Gamma_{N}}\right|^{2} d x+\ell_{D} \int_{\Gamma_{D}} d s+\ell_{N} \int_{\Gamma_{N}} d s+\ell_{K_{D}} \int_{\Sigma_{D}} d s,
$$

where $\ell_{D}, \ell_{N}$ and $\ell_{K_{D}}$ are fixed Lagrange multipliers: $\ell_{D}=2.10^{-2}, \ell_{N}=10^{-3}, \ell_{K_{D}}=10^{-2}$. In the framework of Hadamard's method (see Section 2.2), we consider deformations $\theta$ such that:

$$
\theta \cdot n=0 \text { on } \partial \Omega \text {, and } \theta=0 \text { on } \partial \Omega \backslash\left(\Lambda_{D} \cup \Lambda_{N}\right) .
$$

The numerical resolution of this problem relies on the knowledge of the shape derivatives of the partial mappings $\Gamma_{D} \mapsto J\left(\Gamma_{D}, \Gamma_{N}\right)$ and $\Gamma_{N} \mapsto J\left(\Gamma_{D}, \Gamma_{N}\right)$. In order to accomodate the presence of the transition $\Sigma_{D}:=\overline{\Gamma_{D}} \cap \bar{\Gamma} \subset \Lambda_{D}$ between homogeneous Dirichlet and Neumann boundary conditions, we follow the lead of Section 4 and consider the following approximate counterpart of (5.10):

$$
\inf _{\substack{\Gamma_{D} \subset \Lambda_{D} \\ \Gamma_{N} \subset \Lambda_{N}}} J_{\varepsilon}\left(\Gamma_{D}, \Gamma_{N}\right), \text { where } J_{\varepsilon}\left(\Gamma_{D}, \Gamma_{N}\right):=\int_{\Omega}\left|u_{\Gamma_{D}, \Gamma_{N}, \varepsilon}\right|^{2} d x+\ell_{D} \int_{\Gamma_{D}} d s+\ell_{N} \int_{\Gamma_{N}} d s+\ell_{K_{D}} \int_{\Sigma_{D}} d s
$$

where $u_{\Gamma_{D}, \Gamma_{N}, \varepsilon}$ is the solution in $H^{1}(\Omega)^{3}$ to the system:

$$
\left\{\begin{array}{cl}
-\operatorname{div}\left(A e\left(u_{\Gamma_{D}, \Gamma_{N}, \varepsilon}\right)=0\right. & \text { in } \Omega, \\
\operatorname{Ae}\left(u_{\Gamma_{D}, \Gamma_{N}, \varepsilon}\right)+h_{\varepsilon} u_{\Gamma_{D}, \Gamma_{N}, \varepsilon}=0 & \text { on } \Lambda_{D}, \\
A e\left(u_{\Gamma_{D}, \Gamma_{N}, \varepsilon}\right) n=g_{\text {tool }} & \text { on } \Gamma_{T}, \\
A e\left(u_{\Gamma_{D}, \Gamma_{N}, \varepsilon}\right) n=g & \text { on } \Gamma_{N}, \\
A e\left(u_{\Gamma_{D}, \Gamma_{N}, \varepsilon}\right) n=0 & \text { on } \Gamma \backslash \Lambda_{D},
\end{array}\right.
$$

featuring the interpolation profile $h_{\varepsilon}$ in (4.3).

Thence, the calculation of the shape derivative of $\Gamma_{N} \mapsto J_{\varepsilon}\left(\Gamma_{D}, \Gamma_{N}\right)$ is provided by Section 3.1, or more exactly, the straightforward adaptation of its proof to the present linearized elasticity context. The shape derivative of the smoothed mapping $\Gamma_{D} \mapsto J_{\varepsilon}\left(\Gamma_{D}, \Gamma_{N}\right)$ is calculated exactly as in the proof of Proposition 4.3 (or by using Céa's formal method), and we omit the formula for brevity.

\subsubsection{Numerical representation of the regions $\Gamma_{N}$ and $\Gamma_{D}$}

When it comes to the numerical representation of the optimized subsets $\Gamma_{D}$ and $\Gamma_{N}$ of the lateral boundaries $\Lambda_{D}$ and $\Lambda_{N} \subset \partial \Omega$, we rely on the level set method, pioneered in [48], then introduced in the shape optimization context in $[5,52,55]$.

Let us for instance provide a little more details about the numerical representation of $\Gamma_{D}$ - the same ingredients being used in the case of $\Gamma_{N}$. The region $\Gamma_{D}$ is described as the negative subdomain of a scalar 'level set' function $\phi: \Lambda_{D} \rightarrow \mathbb{R}$, defined on the (planar) lateral boundary $\Lambda_{D}$, that is:

$$
\forall x \in \Lambda_{D}, \begin{cases}\phi(x)<0 & \text { if } x \in \Gamma_{D}, \\ \phi(x)=0 & \text { if } x \in \Sigma_{D}, \\ \phi(x)>0 & \text { otherwise. }\end{cases}
$$

The motion in (pseudo) time of $\Gamma_{D} \equiv \Gamma_{D}(t)$ according to a velocity field with normal component $v(t, x)$ (which in our case is associated to the shape gradient of the optimized functional $J_{\varepsilon}\left(\Gamma_{D}, \Gamma_{N}\right)$ in (5.10)) is then described in terms of an associated level set function $\phi(t, \cdot)$ by the following Hamilton-Jacobi equation:

$$
\frac{\partial \phi}{\partial t}(t, x)+v(t, x)|\nabla \phi(t, x)|=0, \text { for } t>0, x \in \Lambda_{D} .
$$

In practice, (5.12) is discretized in time, and solved on a Cartesian grid of $\Lambda_{D}$ by means of the second-order scheme presented in [51], §6.4.

Last but not least, let us mention that at each iteration of the process, the level set function $\phi$ is reinitialized as the signed distance function $d_{\Gamma_{D}}$ to the actual region $\Gamma_{D}$ by using the fast marching algorithm (see for instance [51], Chap. 8). On the one hand, this operation is well-known to be a key ingredient in the numerical performance of the level set method (see again [51]); on the other hand, the signed distance function $d_{\Gamma_{D}}$ is needed to calculate the coefficient $h_{\varepsilon}$ in (5.11) (see again (4.3)). 


\subsubsection{Numerical application}

Let us now consider a concrete example in the previous context. A tetrahedral mesh of $\Omega$ composed of 45000 vertices is used, and the optimization problem (5.10) is solved for the positions of $\Gamma_{D}$ and $\Gamma_{N}$ while, again, the shape of $\Omega$ itself is unchanged. Relying on the level set method of Section 5.2.2 for representing $\Gamma_{D}$ and $\Gamma_{N}$, we use a standard gradient algorithm based on the knowledge of the shape derivatives of $\Gamma_{D} \mapsto J_{\varepsilon}\left(\Gamma_{D}, \Gamma_{N}\right)$ and $\Gamma_{N} \mapsto J_{\varepsilon}\left(\Gamma_{D}, \Gamma_{N}\right)$; the computation takes about 8 hours and the results are presented on Fig. 7 .

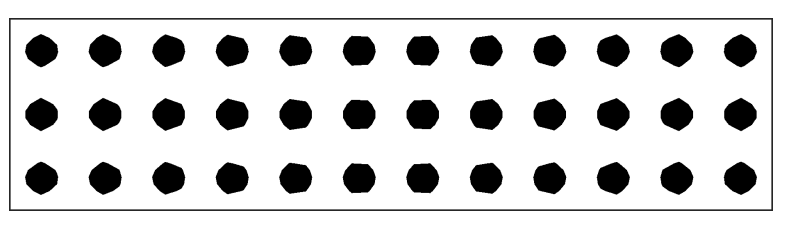

(A) Design of clamps at iteration 1

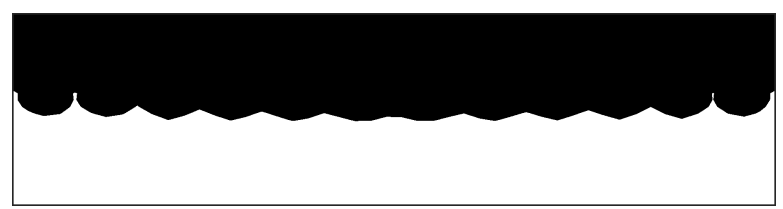

(c) Design of clamps at iteration 20

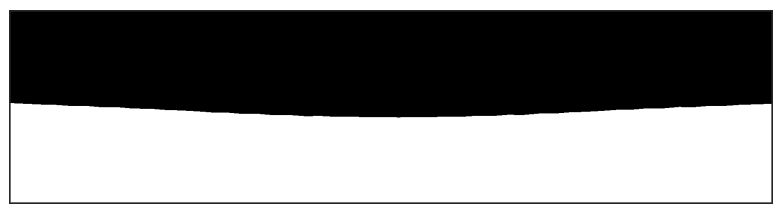

(E) Design of clamps at iteration 100

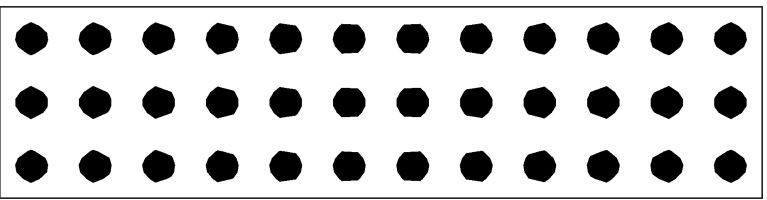

(B) Design of locators at iteration 1

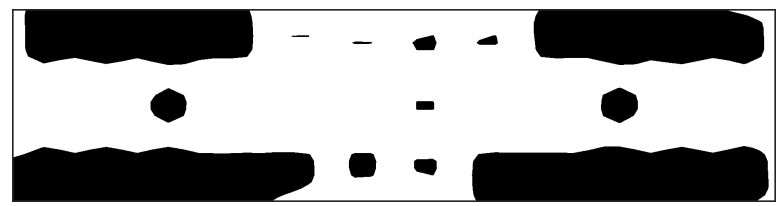

(D) Design of locators at iteration 20

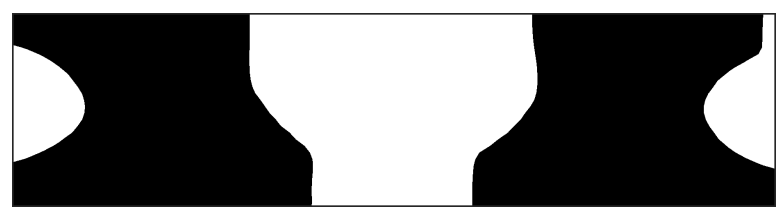

(F) Design of locators at iteration 100

FiguRE 7. Initial, intermediate and optimized designs of clamps and locators in the testcase of Section 5.2.

We notice in particular that the optimized design of the clamps is concentrated under $\Gamma_{T}$ whereas the locators are symmetrically positioned at both ends of the beam. The deformed configurations of the initial and optimized shapes are displayed in Fig. 8.

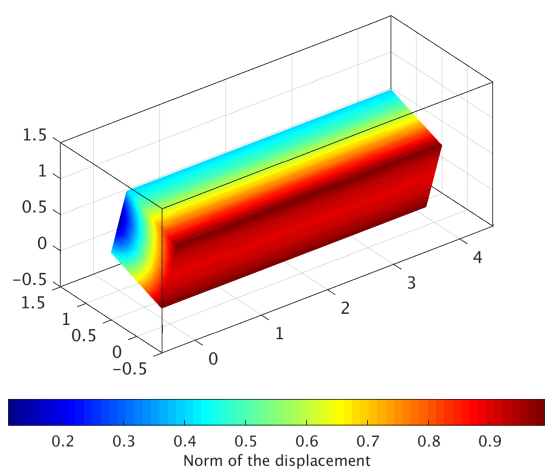

(A) Deformed configuration of $\Omega$ with the initial configuration of clamps and locators

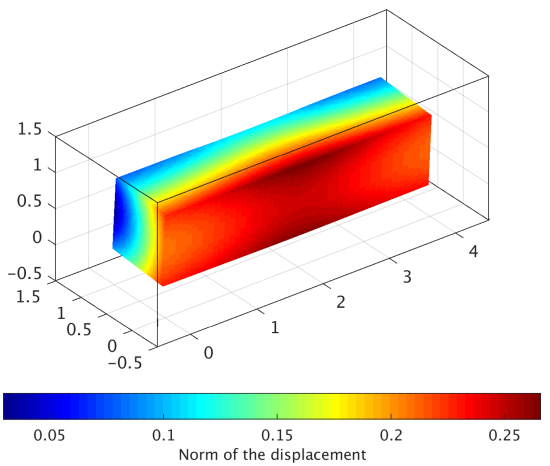

(в) Deformed configuration of $\Omega$ with the optimized configuration of clamps and locators

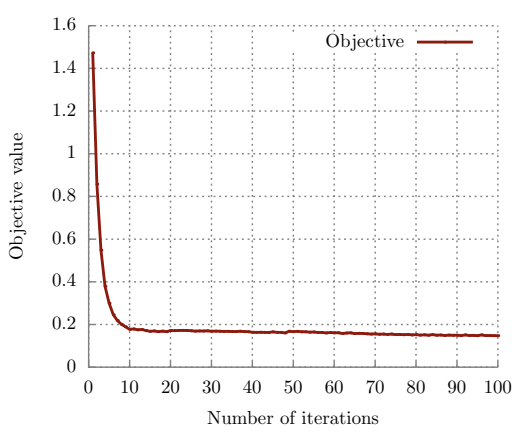

(c) Convergence history

Figure 8. Details of the optimization example of clamps and locators of Section 5.2. 


\subsection{Joint optimization of the shape and the regions supporting different types of boundary conditions}

We finally turn to examples where the shape $\Omega$ of a $2 \mathrm{~d}$ structure is optimized at the same time as the region $\Gamma_{D}$ of its boundary supporting homogeneous Dirichlet boundary conditions. For simplicity, the region $\Gamma_{N}$ supporting inhomogeneous Neumann boundary conditions is fixed, which means that we are exactly in the setting of Sections 3.2 and 4: in all the examples in this subsection, we consider the following shape and topology optimization problem:

$$
\inf _{\substack{\Omega \subset D \\ \Gamma_{D} \subset \Lambda_{D} \cap \partial \Omega}} J(\Omega), \text { where } J(\Omega)=j\left(u_{\Omega}\right)+\ell_{V} \int_{\Omega} d s+\ell_{D} \int_{\Gamma_{D}} d s
$$

is a weighted sum of a case-dependent objective defined from a smooth function $j$, involving the elastic displacement $u_{\Omega}$ of the shape, solution to (5.11), and of constraints on both the volume of shapes, and on the area of the Dirichlet boundary $\Gamma_{D}$ (the latter constraints being enforced by means of fixed Lagrange multipliers $\ell_{V}, \ell_{D}$ ). Notice that in the statement (5.13) of the considered shape optimization problem, we have committed the same abuse of notations as in Section 2.2: $u_{\Omega}$ and $J(\Omega)$ depend on both the overall shape $\Omega$ of the structure and the position $\Gamma_{D}$ of the region supporting homogeneous Dirichlet boundary conditions (the latter being constrained to belong to a fixed region $\Lambda_{D}$ of the computational domain $D$ ), while only the first dependence is explicit.

As regards the numerical setting, the computational domain $D$ is equipped with a fixed mesh. Each shape $\Omega \subset D$ is represented by the level set method, i.e. $\Omega$ is described via a level set function $\phi: D \rightarrow \mathbb{R}$ such that:

$$
\forall x \in D, \begin{cases}\phi(x)<0 & \text { if } x \in \Omega \\ \phi(x)=0 & \text { if } x \in \partial \Omega \\ \phi(x)>0 & \text { otherwise }\end{cases}
$$

see Section 5.2.2 above for more details about the level set method.

Since the shape $\Omega$ is not discretized (it is only known via the datum of a level set function), no computational mesh is available to calculate the elastic displacement $u_{\Omega}$ by means of a standard finite element method. To alleviate this issue, the 'ersatz material trick' (see e.g. [3, 5, 12]) is used to approximate the considered linearized elasticity systems posed on $\Omega$ with systems posed on $D$ as a whole: $u_{\Omega}$ is approximated by the solution $u$ to:

$$
\left\{\begin{array}{cl}
-\operatorname{div}\left(A_{\eta} e(u)\right)=0 & \text { in } D, \\
u=0 & \text { on } \Gamma_{D}, \\
A_{\eta} e(u) n=g & \text { on } \Gamma_{N}, \\
A_{\eta} e(u)=0 & \text { on } \Gamma,
\end{array} \text { where } A_{\eta}(x):=\left\{\begin{array}{cl}
A & \text { if } x \in \Omega, \\
\eta A & \text { otherwise }
\end{array}\right.\right.
$$

and $\eta$ is a small parameter so that the void region $D \backslash \bar{\Omega}$ is filled with a very soft material instead of void (typically, we take $\eta=10^{-3}$ ). In this section the Lamé parameters are still given by (5.6) but using $E=1, \nu=0.3$.

As far as the representation of the optimized part $\Gamma_{D}$ of $\partial \Omega$ is considered, it is constrained to belong to a planar subset $\Lambda_{D}$ of the boundary $\partial D$ in the examples of Sections 5.3.1 and 5.3.2. In this case, it is represented by means of a level set function on a subset of the real line. In Section 5.3.3, the set $\Lambda_{D}$ is a whole region of $D$. Then, $\Gamma_{D}$ is represented by means of a different level set function $\psi: \Lambda_{D} \rightarrow \mathbb{R}$ from that $\phi$ used to represent $\Omega$. Both cases are simple adaptations from the general idea outlined in Section 5.2.2; see also $[57,56]$ about this type of representation.

The same process as before is applied to approximate the transition region $\Sigma_{D}$ between homogeneous Dirichlet and Neumann boundary condition in the formulation of Problem (5.13), and we do not repeat the details for brevity.

\subsubsection{Optimization of the shape of a two-dimensional bridge and its supports}

We first consider the joint optimization of the shape of a two-dimensional bridge $\Omega$ and of the location of its fixations. The situation is that depicted in Fig. 9: $\Omega$ is enclosed inside a two-dimensional computational domain $D$ meshed with 80537 triangles; a unit vertical load is distributed along the upper deck $\Gamma_{N}$, a neighborhood of which is imposed to be part of $\Omega$. We optimize $\Omega$ and the set of fixations $\Gamma_{D}$ (which is 
restrained to a subset $\Lambda_{D}$ of the lower part of $\partial D$ ) with respect to the elastic compliance of the configuration; more precisely, the optimization problem reads as (5.13) with the expressions:

$$
j(u)=\int_{\Gamma_{N}} g \cdot u d s, \quad \ell_{V}=50, \quad \ell_{D}=10 .
$$

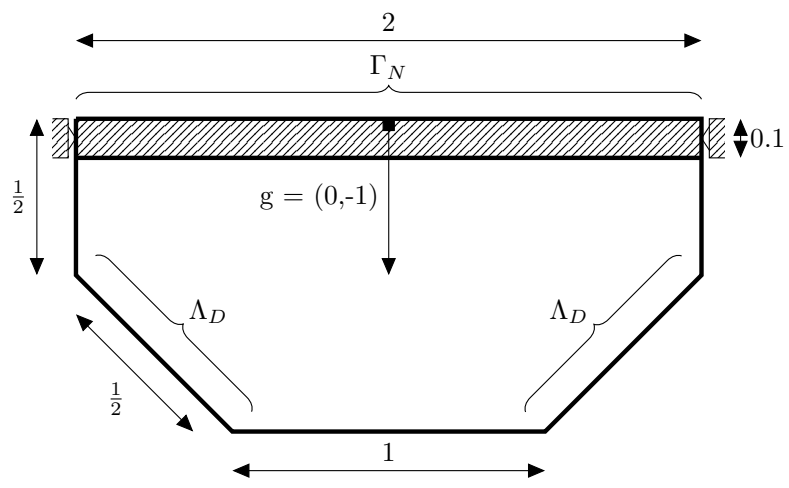

FiguRE 9. Setting of the 2d bridge test-case of Section 5.3.1; the dashed rectangle corresponds to the deck of the bridge, which is a non-optimizable area of $\Omega$.

We perform two optimization experiments, corresponding to different initial states as for $\Omega$ and $\Gamma_{D}$; the results are reported in Fig. 10 and Fig. 11. In particular, we observe very different optimized topologies depending on the initial definition of the fixation region $\Gamma_{D}$.

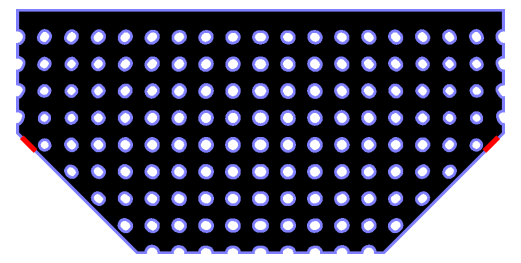

(A) Iteration 1

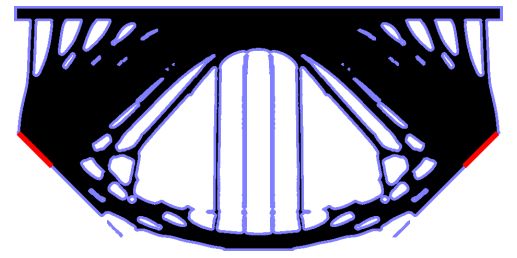

(B) Iteration 25

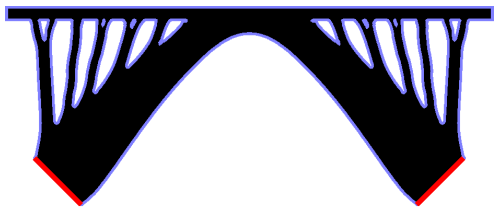

(C) Iteration 100

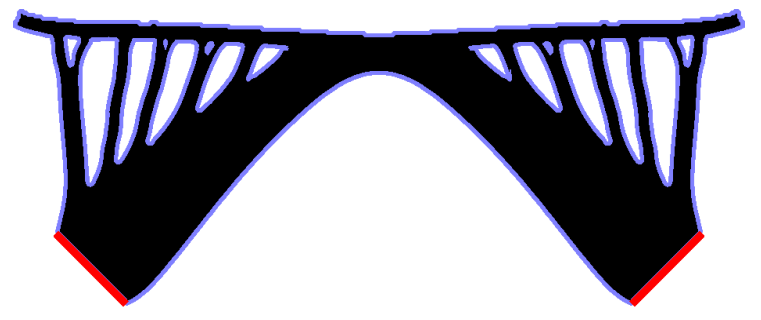

(D) Deformed configuration of the optimized shape

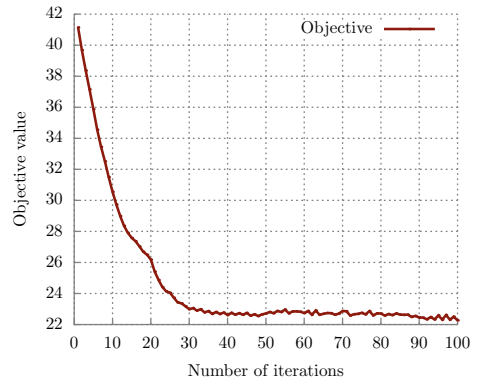

(E) Convergence history

FiguRE 10. Concurrent optimization of the shape and the fixation regions of the bridge of Section 5.3.1, with an initial configuration for $\Gamma_{D}$ composed of two line segments. 


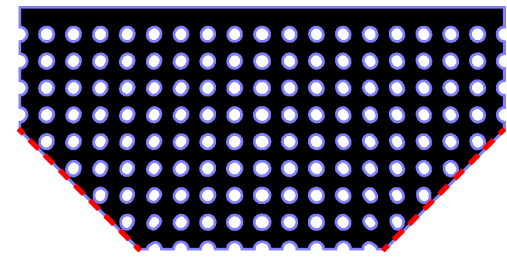

(A) Iteration 1

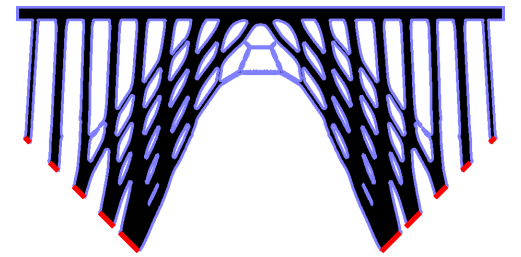

(B) Iteration 25

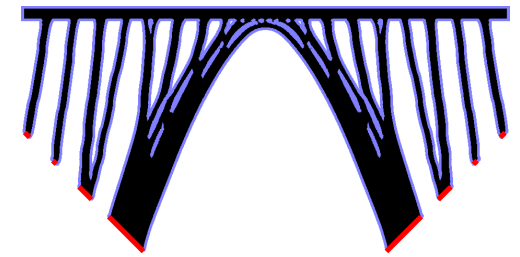

(C) Iteration 100

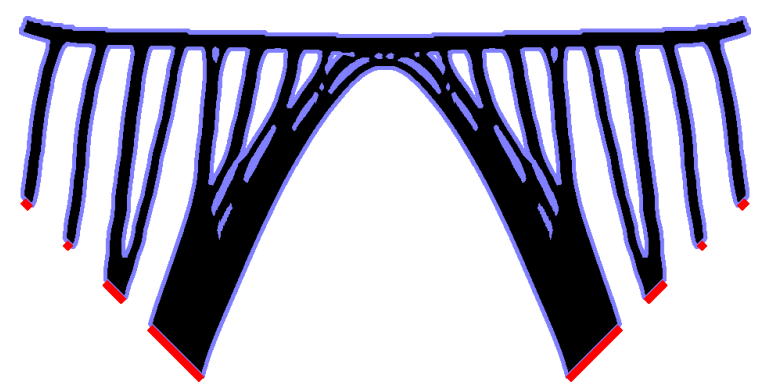

(D) Deformed configuration of the optimized shape

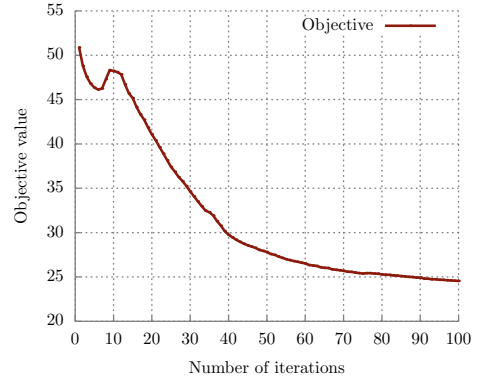

(E) Convergence history

FiguRE 11. Concurrent optimization of the shape and the fixation regions of the bridge of Section 5.3.1, with an initial configuration for $\Gamma_{D}$ composed of 18 line segments.

\subsubsection{Optimization of the shape of a force inverter and of its fixations}

Our second example deals with the optimization of a force inverter mechanism, that is, a device which convert a pulling force into a pushing one. The details of the test-case are presented on Fig. 12: the considered shapes $\Omega$ are contained in a box $D$ meshed with 78408 triangles; they are subjected to a given load $g=(-1,0)$ applied on a non optimizable subset $\Gamma_{N}$ of their left-hand side, and they are attached on another subset $\Gamma_{D}$ of $\partial \Omega$, contained in the upper and lower sides of $\partial D$. In this context, the aim is to optimize the overall shape $\Omega$ and the location of the fixations $\Gamma_{D}$ so that the elastic displacement of $\Omega$ on a non optimizable subset $\Gamma_{T}$ is maximized. The symmetry of the optimized shapes with respect to the horizontal axis is enforced.

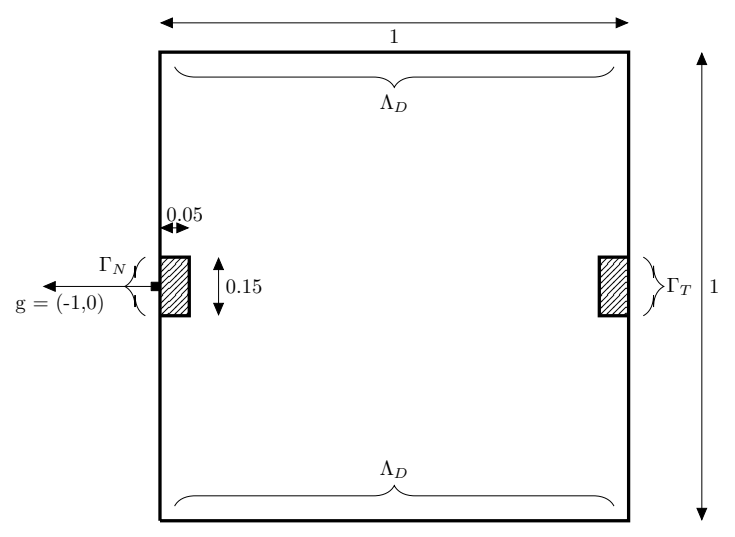

FIGURE 12. Setting of the force inverter test-case of Section 5.3.2. The two dashed rectangles represent non optimizable areas.

More precisely, in the general formulation of the problem (5.13), we set:

$$
j(u)=10^{-1} \int_{\Gamma_{T}}|u-(1,0)|^{2} d s-10^{-3} \int_{\Gamma_{N}} u_{1} d s, \quad \ell_{V}=5 \times 10^{-3}, \quad \ell_{D}=0
$$


where the little penalization on the compliance was added to make it easier to obtain a connected structure.

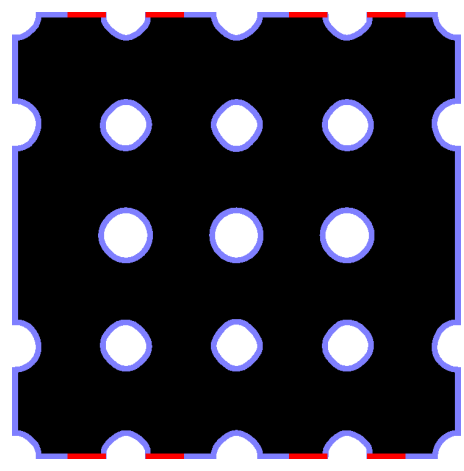

(A) Iteration 1

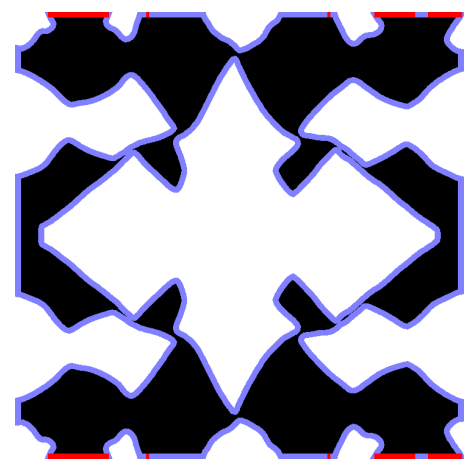

(B) Iteration 50

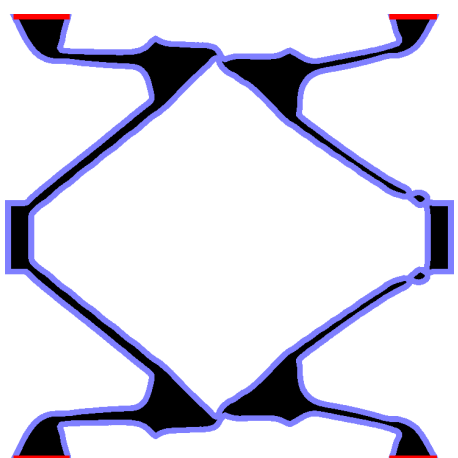

(c) Iteration 100

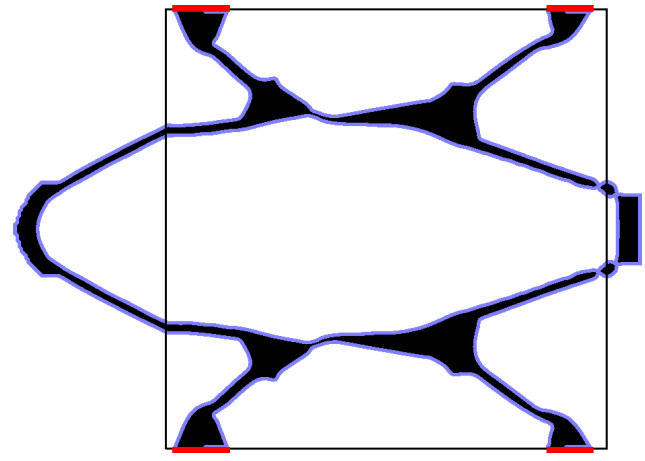

(D) Deformed configuration of the optimized shape

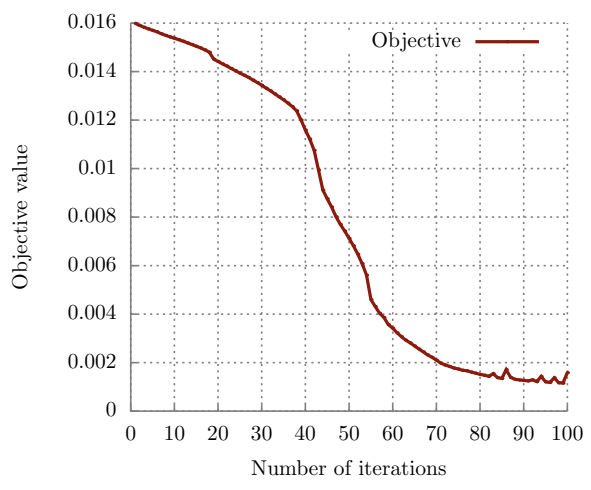

(E) Convergence history

FiguRE 13. Concurrent optimization of the shape and the fixation regions of the force inverter of Section 5.3.2, with an initial configuration for $\Gamma_{D}$ composed of 8 line segments.

\subsubsection{Optimization of the shape and the support regions of a two-dimensional cantilever beam}

Our last example deals with the concurrent optimization of the shape of a classical $2 \mathrm{~d}$ cantilever beam and its fixation zones. The considered shapes $\Omega$ are contained in a fixed computational domain $D$, meshed with 39402 triangles. They are attached on the upper and lower left corners, as well as on a region $\Gamma_{D}$ which is subjected to optimization, and which is constrained to be contained inside a given region $\mathcal{D}_{D} \subset D$. A vertical load $g=(0,-1)$ is applied on a non optimizable subset $\Gamma_{N}$ of the right-hand boundary; see Fig. 15. Notice that, contrary to the previous two examples, the region $\Gamma_{D}$ is not a subset of a region $\Lambda_{D} \subset \partial \mathcal{D}$ but it is allowed to evolve freely inside a region of $\mathcal{D}$. This demands a little adaptation of the framework described above (another level set function is used to identify the region $\Gamma_{D}$ ).

Symmetry with respect to the horizontal axis is imposed on the optimized shape.

All things considered, we consider the optimization problem (5.13) with the expressions:

$$
j(u)=\int_{\Gamma_{T}} g \cdot u d s, \quad \ell_{V}=150, \quad \ell_{D}=0 .
$$

Results are presented on Fig. 16; obviously, the Dirichlet region aims to get as close as possible to the application region of the load $g$. It also tends to concentrate on the top and bottom corners of the region $\mathcal{D}_{D}$, following insofar as possible the principal stress directions of the structure. 


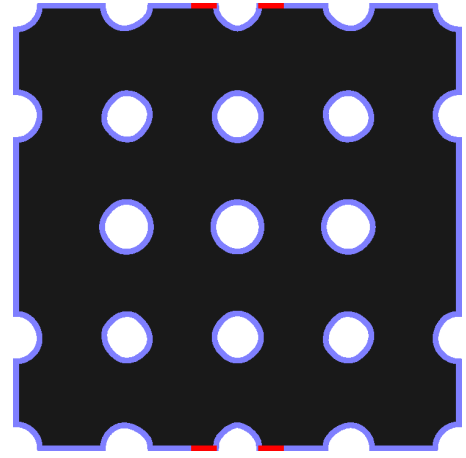

(A) Iteration 1

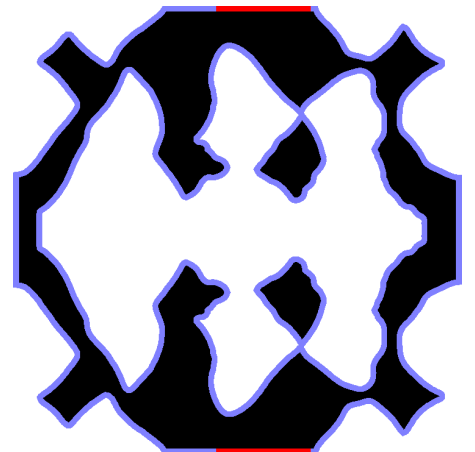

(B) Iteration 50

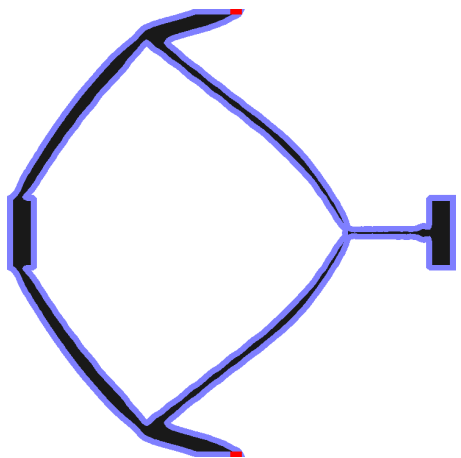

(c) Iteration 300

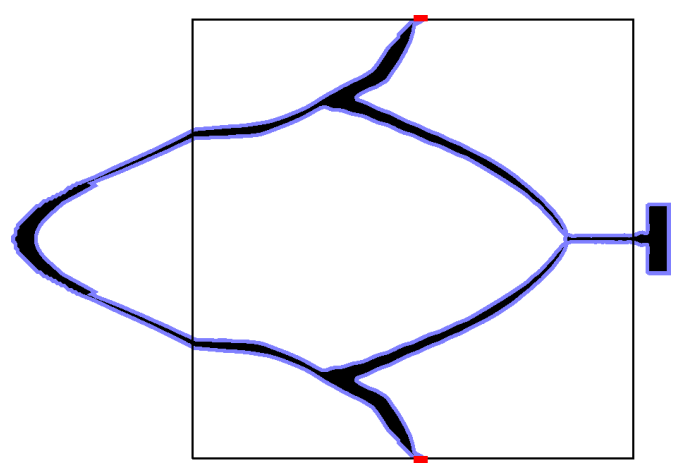

(D) Deformed configuration of the optimized shape

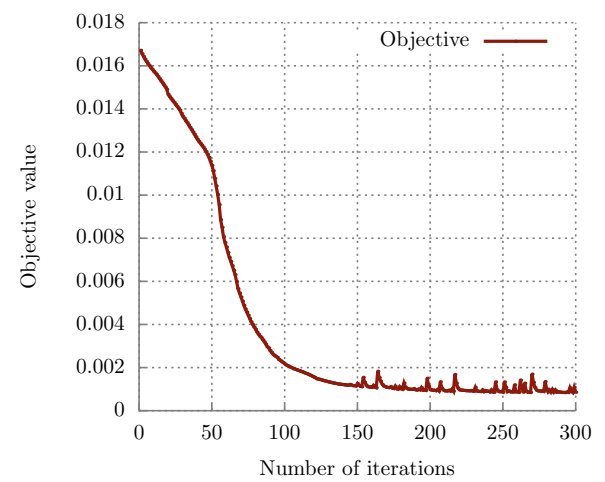

(E) Convergence history

FIGURE 14. Concurrent optimization of the shape and the fixation regions of the force inverter of Section 5.3.2, with an initial configuration for $\Gamma_{D}$ composed of 4 line segments.

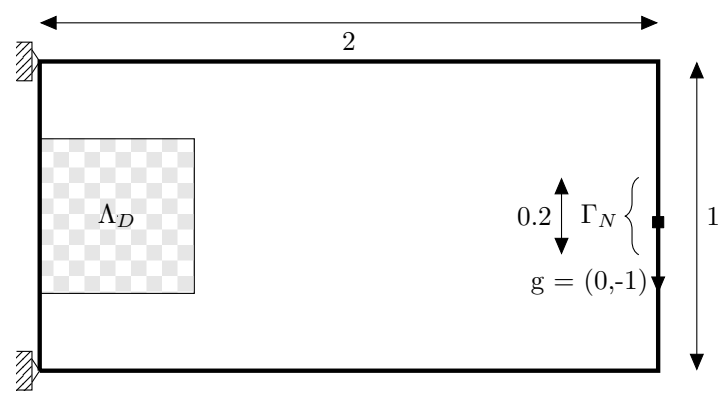

Figure 15. Setting of the 2d cantilever test-case of Section 5.3.3.

\section{Conclusions and Perspectives}

In this article, we have considered the optimization problem of a shape $\Omega$ and of the regions of its boundary supporting different types of boundary conditions in the description of the underlying physical context. Relying on the basic model of a Laplace equation, we have calculated the shape derivative of a quite general objective function $J(\Omega)$ with respect to variations of the 'inhomogeneous Neumann - homogeneous Neumann' and 'homogeneous Dirichlet - homogeneous Neumann' transition zones $\Sigma_{N}$ and $\Sigma_{D}$. Since the second expression is very difficult to use in practice, for it involves a measure of the 'singular' character of the Laplace equation near $\Sigma_{D}$, we have proposed an approximation process to carry out this optimization in 


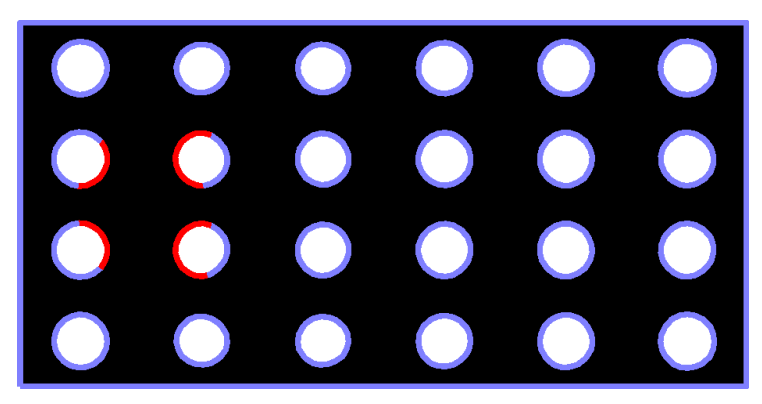

(A) Iteration 1

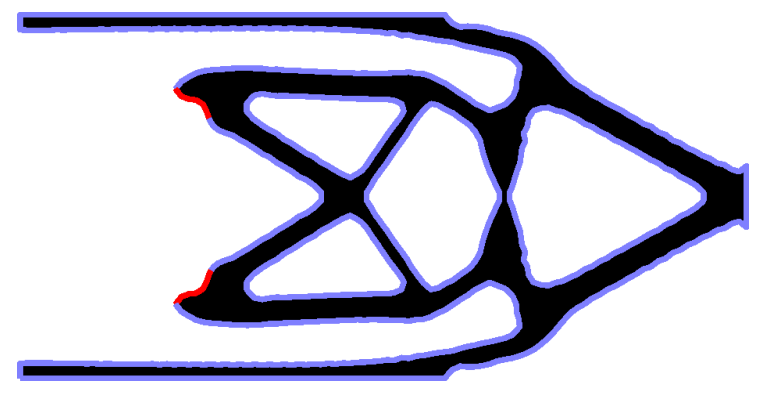

(c) Iteration 50

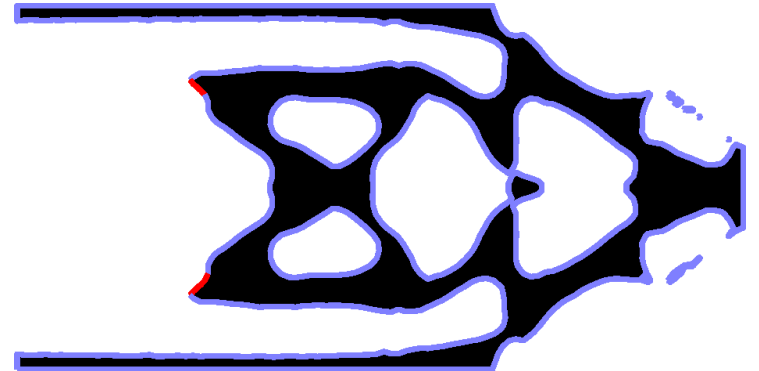

(B) Iteration 25

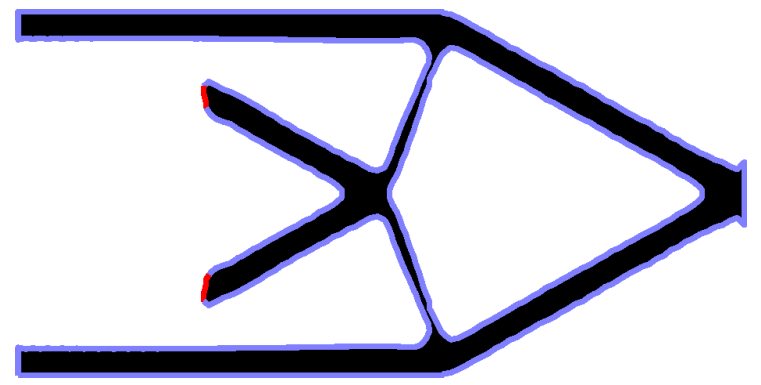

(D) Iteration 100

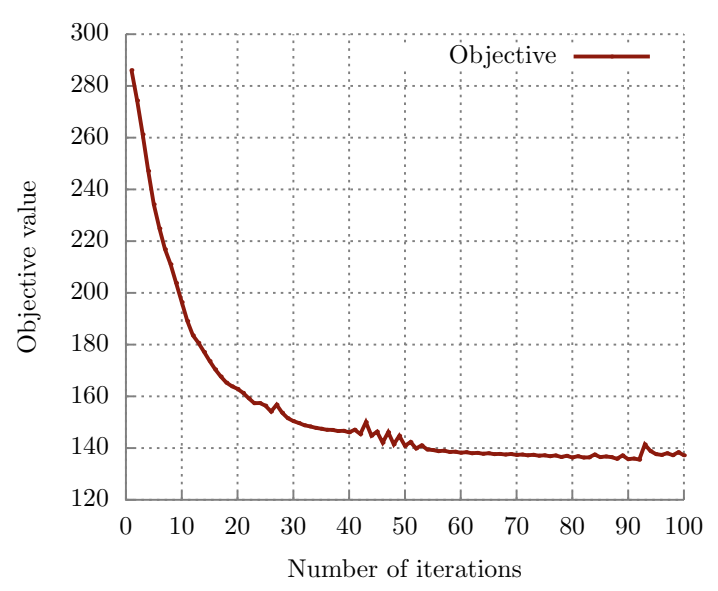

(E) Convergence graph

Figure 16. Concurrent optimization of the shape $\Omega$ and the fixation zones $\Gamma_{D}$ of the twodimensional cantilever of Section 5.3 .3 (the latter are represented using red lines).

practice, which consists in 'smearing' the sharp transition $\Sigma_{D}$ between homogeneous Dirichlet and homogeneous Neumann boundary conditions into a band with small thickness $\varepsilon$ supporting Robin-like boundary conditions. We have verified the numerical efficiency of this process on several application examples involving notably the context of linearized elastic structures in two or three space dimensions. In these examples, we have made the simplifying assumption that $\Sigma_{D}$ belongs to a 'flat' region, and it would be interesting to also conduct the optimization in the case where it is enclosed in a curved surface. Although this setting is already dealt with in our theoretical developments, this is a little more demanding from the numerical point of view: in particular, we would have to apply the level set method on a curved surface, which could for instance involve the so-called 'closest point method'; see for instance [42]. Also, it would be natural and interesting to extend the idea approximate 'singular' transition regions between different types of boundary conditions 
to different physics than those considered in this article (e.g. electromagnetism); let us again mention that it was already used in [24] in the context of the acoustic Helmholtz equations.

Acknowledgements. The authors were partially supported by the ANR Shapo grant. They warmly thank A. Glière and K. Hassan from CEA-LETI, as well as G. Michailidis for fruitful discussions at the origin of this work. The third author gratefully acknowledges the support of the ANR through the project GEOMETRYA, the project COMEDIC and the LabEx PERSYVAL-Lab (ANR-11-LABX-0025-01).

\section{Appendix A. Some facts from tangential calculus}

In this section, we briefly review some facts from tangential calculus which come in handy in several parts of this article; see [33] for a more exhaustive presentation.

Let $\Omega$ be a smooth bounded domain in $\mathbb{R}^{d}$. There exists a tubular neighborhood $U$ of its boundary $\partial \Omega$ such that the projection mapping $p_{\partial \Omega}: U \rightarrow \Gamma$ given by

$$
p_{\partial \Omega}(y)=\text { the unique } x \in \Gamma \text { s.t. }|x-y|=d(y, \Gamma)
$$

is well-defined and smooth; see [7], Th. 3.1. This allows to define smooth extensions of the normal vector field $n$ and of any tangential vector field $\tau: \partial \Omega \rightarrow \mathbb{R}^{d}$ to $U$ via the formulas:

$$
n(y) \equiv n\left(p_{\partial \Omega}(y)\right), \text { and } \tau(y) \equiv \tau\left(p_{\partial \Omega}(y)\right)
$$

respectively. From these notions, we define the mean curvature $\kappa$ of $\partial \Omega$ by $\kappa=\operatorname{div} n$.

In this context, the tangential gradient $\nabla_{\partial \Omega} f$ of a smooth enough function $f: \partial \Omega \rightarrow \mathbb{R}$ is defined by $\nabla_{\partial \Omega} f=\nabla \widetilde{f}-(\nabla \widetilde{f} \cdot n) n$, where $\widetilde{f}$ is any smooth extension of $f$ to an open neighborhood of $\partial \Omega$.

In the same spirit, the tangential divergence $\operatorname{div}_{\partial \Omega} V$ of a smooth vector field $V: \partial \Omega \rightarrow \mathbb{R}^{d}$ is defined by $\operatorname{div}_{\partial \Omega} V:=\operatorname{div} \widetilde{V}-(\nabla \widetilde{V} n) \cdot n$, where $\widetilde{V}$ is any extension of $V$ to an open neighborhood of $\partial \Omega$.

Let us finally recall the following integration by parts formulas on the boundary of smooth domains; see [33], Prop. 5.4.9 for the first point, and [19], §5.5.4 for the second one.

Proposition A.1. Let $\Omega \subset \mathbb{R}^{d}$ be a smooth bounded domain with boundary $\partial \Omega$;

(1) Let $u \in H^{1}(\partial \Omega)$ and $V \in H^{1}(\partial \Omega)^{d}$; then:

$$
\int_{\partial \Omega} \operatorname{div}_{\partial \Omega} V u d s=\int_{\partial \Omega}\left(-V \cdot \nabla_{\partial \Omega} u+\kappa u V \cdot n\right) d s
$$

(2) Let $G$ be a subset of $\partial \Omega$ with smooth boundary $\Sigma$, and denote by $n_{\Sigma}$ its unit normal vector pointing outward $G$ ( $n_{\Sigma}$ is a tangent vector field to $\partial \Omega$ ). Let $u \in H^{1}(\partial \Omega)$ and $V \in H^{1}(\partial \Omega)^{d}$; then:

$$
\int_{G} \operatorname{div}_{\partial \Omega} V u d s=\int_{\Sigma} u V \cdot n_{\Sigma} d \ell+\int_{G}\left(-\nabla_{\partial \Omega} u \cdot V+\kappa u V \cdot n\right) d s
$$

where $d \ell$ denotes integration over the codimension 2 submanifold $\Sigma$ of $\mathbb{R}^{d}$.

\section{REFERENCES}

[1] R. A. Adams and J. J. Fournier, Sobolev spaces, vol. 140, Academic press, 2003.

[2] S. Agmon, A. Douglis, And L. Nirenberg, Estimates near the boundary for solutions of elliptic partial differential equations satisfying general boundary conditions. $i$, Communications on pure and applied mathematics, 12 (1959), pp. 623727.

[3] G. Allaire, Shape Optimization by the Homogenization Method, vol. 146, Springer Science \& Business Media, 2001.

[4] G. Allaire, C. Dapogny, G. Delgado, and G. Michailidis, Multi-phase structural optimization via a level set method, ESAIM: Control, Optimisation and Calculus of Variations, 20 (2014), pp. 576-611.

[5] G. Allaire, F. Jouve, And A.-M. Toader, Structural optimization using sensitivity analysis and a level-set method, Journal of computational physics, 194 (2004), pp. 363-393.

[6] G. Allaire and M. Schoenauer, Conception optimale de structures, vol. 58, Springer, 2007.

[7] L. Ambrosio And H. M. Soner, Level set approach to mean curvature flow in arbitrary codimension, Journal of Differential Geometry, (1994), pp. 693-737.

[8] H. Azegami, K. Ohtsuka, And M. Kimura, Shape derivative of cost function for singular point: Evaluation by the generalized $j$ integral, JSIAM Letters, 6 (2014), pp. 29-32.

[9] I. BABUŠKA AND M. SURI, The $p$-and hp versions of the finite element method, an overview, Computer Methods in Applied Mechanics and Engineering, 80 (1990), pp. 5-26. 
[10] M. Barone And D. Caulk, Optimal arrangement of holes in a two-dimensional heat conductor by a special boundary integral method, International Journal for Numerical Methods in Engineering, 18 (1982), pp. 675-685.

[11] H. BAwA, Manufacturing processes-II, vol. 2, Tata McGraw-Hill Education, 2004.

[12] M. P. Bendsoe And O. Sigmund, Topology optimization: theory, methods, and applications, Springer Science \& Business Media, 2013.

[13] H. Brezis, Functional analysis, Sobolev spaces and partial differential equations, Springer Science \& Business Media, 2010.

[14] M. BuRger, A framework for the construction of level set methods for shape optimization and reconstruction, Interfaces and Free boundaries, 5 (2003), pp. 301-329.

[15] J. CÉA, Conception optimale ou identification de formes, calcul rapide de la dérivée directionnelle de la fonction coût, ESAIM: Mathematical Modelling and Numerical Analysis, 20 (1986), pp. 371-402.

[16] M. Costabel, Boundary integral operators on lipschitz domains: elementary results, SIAM Journal on Mathematical Analysis, 19 (1988), pp. 613-626.

[17] M. Costabel and M. Dauge, A singularly mixed boundary value problem, Communications in Partial Differential Equations, 21 (1996), pp. 1919-1949.

[18] M. Costabel and E. Stephan, Curvature terms in the asymptotic expansions for solutions of boundary integral equations on curved polygons, The Journal of Integral Equations, (1983), pp. 353-371.

[19] C. Dapogny, Shape optimization, level set methods on unstructured meshes and mesh evolution, PhD thesis, Paris 6, 2013.

[20] M. DAuge, Elliptic boundary value problems on corner domains: smoothness and asymptotics of solutions, vol. 1341, Springer, 2006

[21] F. De Gournay, Velocity extension for the level-set method and multiple eigenvalues in shape optimization, SIAM journal on control and optimization, 45 (2006), pp. 343-367.

[22] J. D. Deaton and R. V. Grandhi, A survey of structural and multidisciplinary continuum topology optimization: post 2000, Structural and Multidisciplinary Optimization, 49 (2014), pp. 1-38.

[23] M. C. Delfour And J.-P. ZolÉsio, Shapes and geometries: metrics, analysis, differential calculus, and optimization, SIAM, 2011.

[24] J. Desai, A. Faure, G. Michailidis, G. Parry, and R. Estevez, Topology optimization in acoustics and elasto-acoustics via a level-set method, submitted, (2017).

[25] M. P. Do Carmo and J. Flaherty Francis, Riemannian geometry, vol. 115, Birkhäuser Boston, 1992.

[26] P. D. DunNing AND H. A. KIM, Introducing the sequential linear programming level-set method for topology optimization, Structural and Multidisciplinary Optimization, 51 (2015), pp. 631-643.

[27] M. Elliotis, G. Georgiou, and C. Xenophontos, Solving laplacian problems with boundary singularities: a comparison of a singular function boundary integral method with the $p / h p$ version of the finite element method, Applied Mathematics and Computation, 169 (2005), pp. 485-499.

[28] G. Fremiot And J. Sokolowski, Shape sensitivity analysis of problems with singularities, Lecture notes in pure and applied mathematics, (2001), pp. 255-276.

[29] M. Giacomini, O. Pantz, and K. Trabelsi, Volumetric expressions of the shape gradient of the compliance in structural shape optimization, arXiv preprint arXiv:1701.05762, (2017).

[30] P. Grisvard, Elliptic problems in nonsmooth domains, SIAM, 2011.

[31] J. HASLINGER AND M. R.A.E., Introduction to shape optimization: theory, approximation, and computation, vol. 7, Siam, 2003.

[32] F. Hecht, New development in freefem++, J. Numer. Math., 20 (2012), pp. 251-265.

[33] A. Henrot and M. Pierre, Variation et optimisation de formes: une analyse géométrique, vol. 48, Springer Science \& Business Media, 2006.

[34] A. Henrot and J. SokoŁowski, Mathematical challenges in shape optimization, Control and Cybernetics, 34 (2005), pp. $37-57$.

[35] R. Hiptmair, A. Paganini, and S. Sargheini, Comparison of approximate shape gradients, BIT Numerical Mathematics, 55 (2015), pp. 459-485.

[36] N. KAYA, Machining fixture locating and clamping position optimization using genetic algorithms, Computers in Industry, 57 (2006), pp. 112-120.

[37] V. A. Kozlov, V. Mazia, And J. Rossmann, Elliptic boundary value problems in domains with point singularities, vol. 52, American Mathematical Soc., 1997.

[38] S. LANG, Fundamentals of differential geometry, vol. 191, Springer Science \& Business Media, 2012.

[39] Z.-C. Li And T.-T. Lu, Singularities and treatments of elliptic boundary value problems, Mathematical and Computer Modelling, 31 (2000), pp. 97-145.

[40] J.-L. Lions and E. Magenes, Problèmes aux limites non homogènes et applications. volume i, (1968).

[41] J. MA, M. Y. WANG, AND X. Zhu, Compliant fixture layout design using topology optimization method, in Robotics and Automation (ICRA), 2011 IEEE International Conference on, IEEE, 2011, pp. 3757-3763.

[42] C. B. Macdonald And S. J. Ruuth, Level set equations on surfaces via the closest point method, Journal of Scientific Computing, 35 (2008), pp. 219-240.

[43] J.-J. MARigo, Plasticité et rupture, 2016.

[44] W. C. H. MCLean, Strongly elliptic systems and boundary integral equations, Cambridge university press, 2000.

[45] B. Mohammadi and O. Pironneau, Applied shape optimization for fluids, Oxford university press, 2010. 
[46] F. Murat and J. Simon, Sur le contrôle par un domaine géométrique, Preprint of the Laboratoire d'Analyse Numérique (76015), (1976), p. 222 pages.

[47] K. OhtsukA, Shape optimization by generalized j-integral in poisson's equation with a mixed boundary condition, in International Conference Continuum Mechanics Focusing on Singularities, Springer, 2016, pp. 73-83.

[48] S. Osher And J. A. Sethian, Fronts propagating with curvature-dependent speed: algorithms based on hamilton-jacobi formulations, Journal of computational physics, 79 (1988), pp. 12-49.

[49] L. Rakotondrainibe, Modélisation mécanique d'une "Face Accessoire Assemblée" et optimisation d'un modèle simplifié., PhD thesis, Ecole Polytechnique X, 2017.

[50] S. Selvakumar, K. Arulshri, K. Padmanaban, and K. Sasikumar, Design and optimization of machining fixture layout using ann and doe, The International Journal of Advanced Manufacturing Technology, 65 (2013), pp. 1573-1586.

[51] J. A. SEthian, Level set methods and fast marching methods: evolving interfaces in computational geometry, fluid mechanics, computer vision, and materials science, vol. 3, Cambridge university press, 1999.

[52] J. A. Sethian and A. Wiegmann, Structural boundary design via level set and immersed interface methods, Journal of computational physics, 163 (2000), pp. 489-528.

[53] J. Sokolowski AND J.-P. Zolesio, Introduction to shape optimization, Springer, 1992.

[54] R. Subbarao And P. Meer, Nonlinear mean shift over riemannian manifolds, International Journal of Computer Vision, 84 (2009), pp. 1-20.

[55] M. Y. WANG, X. WANG, AND D. GuO, A level set method for structural topology optimization, Computer methods in applied mechanics and engineering, 192 (2003), pp. 227-246.

[56] Q. XIA AND T. SHI, Topology optimization of compliant mechanism and its support through a level set method, Computer Methods in Applied Mechanics and Engineering, 305 (2016), pp. 359-375.

[57] Q. XIA, M. Y. WANG, AND T. SHI, A level set method for shape and topology optimization of both structure and support of continuum structures, Computer Methods in Applied Mechanics and Engineering, 272 (2014), pp. 340-353.

[58] W. Zhang, L. ZhaO, And S. CAI, Shape optimization of dirichlet boundaries based on weighted b-spline finite cell method and level-set function, Computer Methods in Applied Mechanics and Engineering, 294 (2015), pp. 359-383. 